\title{
ALGUNS RESULTADOS SOBRE OS FLUIDOS MICROPOLARES NÃO-HOMOGÊNEOS
}

\author{
Mariano Poblete Cantellano
}

TESE APRESENTADA

$\mathrm{AO}$

INSTITUTO DE MATEMÁTICA E ESTATÍSTICA

DA

UNIVERSIDADE DE SÃO PAULO

PARA

OBTENÇÃO DO GRAU

$\mathrm{DE}$

DOUTOR EM MATEMÁTICA APLICADA

Área de Concentração: Equações Diferenciais Parciais

Orientador: Prof. Dr. Marko Antonio Rojas Medar

DMA - IMECC - UNICAMP

- São Paulo, Agosto de 2001 - 
Este exemplar corresponde à redação final da dissertação apresentada por Edson Tsukimoto, devidamente corrigida e aprovada pela Comissão Julgadora

São Paulo, agosto de 2001

\section{Comissão Julgadora}

- Prof. Dr Marko Antonio Rojas-Medar (Orientador) - IMECC-UNUICAMP.

- Prof. Dr. Pedro Paulo Serpa Schirmer - IME-USP.

- Prof. Dr.Adalberto Panobianco Bergamasco - UFSCAR.

- Prof. Dr.José Luiz Boldrini - IMECC-UNICAMP.

- Prof. Dr Marcelo Martins dos Santos - IMECC-UNICAMP. 


\section{Agradecimentos}

Gostaria de prestar aqui meus agradecimentos ás pessoas que foram importantes durante o o curso de doutoramento, profesores, amigos e colegas em geral e funcionarios da biblioteca (Silvia e Max pela simpatia).

Agradeço ao meu Orientador Marko A. Rojas-Medar pela atenção prestada durante o trabalho de tese. Além disso, agradeço pelo companheirismo aos amigos que fiz durante minha estadia na UNICAMP, especialmente Marcio, Jurilev, Gabriela, Rogerio, Hermes, Roberto. Muito especialmente a meus colegas e amigos do IME (USP) Brasileiros, Uruguaios, Argentinos, Colombianos, Peruanos, Chilenos, espanhois e alemaẽs. Entre eles, gostaria de nombrar a Jairo, Sandra, Claudia e Juán, Raúl, e meu querido amigo Gonzalo junto com sua esposa e o pequeno Alexis com quienes compartilhe boas comidas Chilenas. Outros amigos e compatriotas como Marco Antonio e familia, Mauricio e Familia sou grato pelos bons momentos que compartilhiamos.

Agradeço aos profesores do IME pela formação recibida e a CAPES pelo apoio financeiro recido durante mi estadia no Brasil.

Finalmente, um agradecimento carinhoso a minha esposa e filhos por toda a paciência durante o tempo que esteve no Brasil. 


\section{Resumo}

Neste trabalho estudamos algumas questões matemáticas relacionadas com os fluidos assimétricos não-homogêneos. Apresentamos um resultado de existência e unicidade de soluções fortes para este sistema sobre um domínio qualquer tridimensional não-limitado. Também, apresentamos um resultado de existência de soluções fracas reprodutivas. Finalmente, apresentamos um resultado de existência de soluções fracas para uma desigualdade variacional associada ao sistema de equações dos fluidos assimétricos não-homogêneos.

\section{Abstract}

In this work we study some mathematical questions related with assymetric nonhomogeneous fluids. We give a result on the existence and uniqueness of strong solutions on unbounded domains. Also, we give a result on the existence of reproductive weak solutions. And, finally we give a result on the existence of weak solutions for variational inequalities associated with the assymetric nonhomogeneous fluids. 


\section{Sumário}

Introdução 1

0.1 Algumas questões físicas do sistema FIMAN . . . . . . . . . . . 2

0.2 Resultados teóricos conhecidos . . . . . . . . . . . . . 6

0.2.1 Fluidos micropolares com densidade constante . . . . . . . 6 6

0.2 .2 Fluidos não-homogêneos assimétricos . . . . . . . . . . . . . 7

1 Preliminares 9

1.1 Espaços de Sobolev com valores reais . . . . . . . . . . . . . . . . . . 9

1.2 Espaços de Sobolev com valores num espaço de Banach . . . . . . . . 13

1.3 Produtos contínuos entre espaços de Sobolev reais em dimensão 3 . . 16

1.4 Resultado de compacidade . . . . . . . . . . . . . . . . . . 17

1.5 Espaços de funções com "divergente nulo" . . . . . . . . . . . . . . 18

2 Existência e unicidade de soluções fortes num domínio não-limitado 20

2.1 Introdução e resultado principal . . . . . . . . . . . . . . . 20

2.2 Resultados auxiliares . . . . . . . . . . . . . . . . 23

2.3 Aproximações auxiliares . . . . . . . . . . . . . . . . . . 37

2.4 Demonstração do teorema principal . . . . . . . . . . . . . . 46

3 Soluções periódicas dos fluidos incompressíveis assimétricos nãohomogêneos $\quad \mathbf{5 6}$

3.1 Introdução . . . . . . . . . . . . . . . . . . . . 56

3.2 Definição e apresentação do problema . . . . . . . . . . . . . 58

3.3 Problema auxiliar e regularização elíptica . . . . . . . . . . . . 60

3.3.1 Estimativas a priori para a densidade . . . . . . . . . 6 63

3.4 Soluções reprodutivas do problema regularizado . . . . . . . . . . . 64

3.5 Demonstração do resultado principal . . . . . . . . . . . . . 73

4 Equações variacionais $\quad \mathbf{8 3}$

4.1 Introdução . . . . . . . . . . . . . . . . . . . . . . 83

4.2 Colocação do problema das formulações variacionais . . . . . . . . . . 84

4.3 Existência de soluções fracas . . . . . . . . . . . . . . . . . . . 92

4.3.1 Problema auxiliar . . . . . . . . . . . . . . . . . 99 93

4.3.2 Estimativas a priori . . . . . . . . . . . . . . . . . 997

4.4 Estimativas no tempo e passagem ao limite. . . . . . . . . . . . 101

4.5 Problemas em aberto . . . . . . . . . . . . . . . 111

Referências 


\section{Notações}

\section{Notação vetorial e matricial}

$\mathbb{N}$ : Conjunto dos números naturais

$\alpha \in \mathbb{N}^{N}:$ Multi-índice de "comprimento" $|\alpha|=\alpha_{1}+\alpha_{2}+\cdots+\alpha_{N}$

$\mathbb{R}$ : Números reais

$|x|$ : Valor absoluto de $x \in \mathbb{R}$

$\mathbb{R}^{N}:$ Espaço euclideano de dimensão $N \in \mathbb{N}$

$X \cdot Y$ : Produto escalar euclideano dos vetores $X, Y \in \mathbb{R}^{N}$

$|X|=(X \cdot X)^{1 / 2}:$ Norma euclideana de $X \in \mathbb{R}^{n}$

$X \otimes Y:$ Matriz (produto tensorial) de ordem $N \times N$, cujo coeficiente $(i, j)$ é $x_{j} y_{j}$, onde $X=\left(x_{1}, x_{2}, \ldots, x_{N}\right)$ e $Y=\left(y_{1}, y_{2}, \ldots, y_{N}\right)$

\section{Notações geométricas}

$\Omega$ : Aberto de $\mathbb{R}^{N}$, de medida de Lebesgue $|\Omega|$

$\partial \Omega$ ou $\Gamma$ : Fronteira de $\Omega$

$n=n(x)$ : Vetor normal unitário no ponto $x \in \partial \Omega$ orientado para o exterior de $\Omega$ 


$$
\begin{aligned}
& Q_{T}=\Omega \times(0, T) \\
& \Sigma_{T}=\partial \Omega \times(0, T)
\end{aligned}
$$

\section{Operadores diferenciais}

$\frac{\partial^{\alpha}}{\partial x^{\alpha}}=\frac{\partial^{|\alpha|}}{\partial x_{1}^{\alpha_{1}} \cdots \partial x_{N}^{\alpha_{N}}}$ : Derivada parcial associada ao multi-índice

$\alpha=\left(\alpha_{1}, \alpha_{2}, \ldots, \alpha_{N}\right) \in \mathbb{N}^{N}$

$\nabla=\left(\frac{\partial}{\partial x_{1}}, \frac{\partial}{\partial x_{2}}, \ldots, \frac{\partial}{\partial x_{N}}\right):$ Operador gradiente; para uma função escalar $\rho$, $\nabla \rho$ é um vetor de $i$-ésima componente $\frac{\partial \rho}{\partial x_{i}}$; para uma função vetorial $u=$ $\left(u_{1}, \ldots, u_{N}\right), \nabla u$ é uma matriz cuja componente $(i, j)$ é $\frac{\partial u_{i}}{\partial x_{j}}$

$\Delta=\sum_{i=1}^{N} \frac{\partial^{2}}{\partial x_{i}^{2}}:$ Operador de Laplace

$\operatorname{div} \equiv \nabla \cdot:$ Operador divergente; para uma função vetorial $u, \operatorname{div} u=\nabla \cdot u=$ $\sum_{i=1}^{N} \frac{\partial u_{i}}{\partial x_{i}}$

$(\rho u \cdot \nabla) u$ : Campo vetorial da $i$-ésima componente $\sum_{j=1}^{N} \rho u_{j} \frac{\partial u_{i}}{\partial x_{j}}$

$\rho u u \equiv \rho u \otimes u$ : Campo matricial com componentes $(i, j)$ igual a $\rho u_{i} u_{j}$

rot: Operador rotacional; para uma função vetorial $u$,

$$
\operatorname{rot} u=\left(\frac{\partial u_{3}}{\partial x_{2}}-\frac{\partial u_{2}}{\partial x_{3}}, \frac{\partial u_{1}}{\partial x_{3}}-\frac{\partial u_{3}}{\partial x_{1}}, \frac{\partial u_{2}}{\partial x_{1}}-\frac{\partial u_{1}}{\partial x_{2}}\right)
$$

\section{Espaços de funções a valores reais}

$C^{m}(\Omega)(m \in \mathbb{N}$ ou $m=+\infty)$ : Espaço vetorial das funções de $\Omega \mapsto \mathbb{R}, m$ vezes continuamente diferenciáveis (para $m=0$, são funções contínuas)

$D(\Omega)$ : Espaço vetorial das funções test, isto é, de classe $C^{\infty}$ e de suporte compacto contido em $\Omega$ 
$D^{\prime}(\Omega)$ : Espaço das distribuições sobre $\Omega$, isto é, aplicações lineares contínuas de $D(\Omega)$ em $\mathbb{R}$ (para a topologia limite indutivo usual de $D(\Omega)$ )

$L^{p}(\Omega)$ : Espaço de Banach das funções (classes de) de $\Omega$ em $\mathbb{R}$, mensuráveis e $p$ integráveis

$W_{p}^{m}(\Omega)$ ( $m$ natural): Espaço das (classes de) funções de $L^{p}(\Omega)$, cujas derivadas em $D^{\prime}(\Omega)$ de ordem $\leq m$ pertencem a $L^{p}(\Omega)$. São espaços de Banach.

\section{Espaços de funções com valores num espaço de Banach}

$\|\cdot\|_{B}$ : Norma em um Espaço de Banach $B$

$B^{\prime}$ : Dual topológico de $B$

$\langle\cdot, \cdot\rangle_{B^{\prime} B}$ : Produto dualidade entre $B^{\prime}$ e $B$. Às vezes escrevemos simplesmente $\langle$,

0,T: Tempo inicial e final de observação

$L^{p}(0, T ; B)$ : Espaço de Banach das (classes de) funções $f:[0, T] \rightarrow B$ mensuráveis, tais que a função $t \in[0, T] \rightarrow\|f(t)\|_{B}$ é $p$-integrável

$C^{m}([0, T] ; B)$ : Espaço de Banach das funções $f:[0, T] \mapsto B, m$ vezes continuamente diferenciáveis (em relação à topologia forte de $B$ )

$D(0, T ; B)$ : Espaço vetorial das funções $f:[0, T] \mapsto B$ de classe $C^{\infty}$ e de suporte compacto contido em $(0, T)$

$D^{\prime}(0, T ; B)=\mathcal{L}(D(0, T) ; B)$ : Espaço das distribuições com valores em $B$, isto é, aplicações lineares "contínuas" de $D(0, T)$ em $B$, para a topologia limite indutivo usual de $D(0, T)$ e a topologia forte de $B$ 


\section{Abreviaturas}

q.t.p: Quasi em toda parte (geralmente com respeito à medida de Lebesgue)

Edp: Equações a derivadas parciais

7. Tipos de aplicações

$\mapsto$ : Aplicação ou função

$\hookrightarrow$ : Imersão algébrica e topológica (imersão contínua)

$\longmapsto:$ Imersão compacta 


\section{Introdução}

O objetivo deste trabalho é abordar o estudo sobre a existência e unicidade de soluções fortes e fracas para um sistema de Equações dos Fluidos Micropolares Assimétricos Não-Homogêneos (FIMAN). Mas, antes de comentar resultados sobre estas equações, apresentaremos este sistema de Edp e tentaremos descrever "grosso modo" suas principais características físicas. As notações utilizadas em relação à geometria dos domínios, operadores diferenciais, espaços funcionais, etc., são as de hábito em equações diferenciais parciais e para maior precisão e esclarecimentos foram listados na "Folha de Notações" páginas i - iv. Para escrever as equações do movimento de um FIMAN, denotamos as funções desconhecidas por $u(x, t)$, $w(x, t) \in \mathbb{R}^{3}, \rho(x, t), p(x, t) \in \mathbb{R}$, definidas sobre o cilindro $Q_{T}=\Omega \times(0, T)$.

As duas primeiras funções (vetoriais) denotam a velocidade e a velocidade angular de rotações das partículas dos fluidos, respectivamente. As funções escalares denotam a densidade e a pressão, respectivamente. Assim sendo, a descrição matemática do comportamento dinâmico de um fluido que ocupa uma região $\Omega \subset \mathbb{R}^{3}$ durante um intervalo de tempo $[0, T]$ é escrita como

$$
\begin{aligned}
& \rho\left\{\frac{\partial u}{\partial t}+(u \cdot \nabla) u\right\}-\left(\mu+\mu_{r}\right) \Delta u+\nabla p=\rho f+2 \mu_{r} \operatorname{rot} w, \\
& \operatorname{div} u=0, \\
& \rho\left\{\frac{\partial w}{\partial t}+u \cdot \nabla w\right\}-\nu \Delta w-\gamma \nabla \operatorname{div} w+4 \mu_{r} w=\rho g+2 \mu_{r} \operatorname{rot} u, \\
& \frac{\partial \rho}{\partial t}+(u \cdot \nabla) \rho=0,
\end{aligned}
$$




$$
\begin{aligned}
& u \equiv 0, w \equiv 0 \text { sobre } \Sigma_{T}=\partial \Omega \times(0, T), \\
& u(x, 0)=u_{0}(x), w(x, 0)=w_{0}(x) \text { em } \Omega, \\
& \rho(x, 0)=\rho_{0}(x) \text { em } \Omega .
\end{aligned}
$$

As constantes positivas $\nu=C_{a}+C_{d}, \gamma=C_{0}+C_{d}-C_{a}, \mu, \mu_{r}$, denotam viscosidades envolvidas no fenômeno. Mais precisamente, as constantes $\mu, \mu_{r}, C_{0}, C_{a}, C_{d}$ caracterizam as propriedades isotrópicas do fluido. A constante $\mu$ é a viscosidade Newtoniana usual e $\mu_{r}, C_{0}, C_{a}, C_{d}$ são constantes positivas relacionadas com a assimetria do tensor de tensões e satisfazem a relação $C_{0}+C_{d}>C_{a}$. As funções $f, g$ denotam fontes externas e estão definidas sobre $Q_{T}$ a valores em $\mathbb{R}^{3}$.

As equações $(0.1)$ - (0.4) são satisfeitas no cilindro $Q_{T}$, onde $(0.1),(0.3)$ representam as leis de conservação do momentum linear e de momentum angular, respectivamente. A condição (0.2) significa que o fluido é incompressível, isto é, o volume ocupado por um conjunto de partículas que se deslocam ao longo das trajetórias não varia com o tempo. A equação (0.4) é a conhecida equação da conservação de massa ou equação da continuidade. Finalmente, (0.5) denota condições de fronteira tipo Dirichlet, $(0.6),(0.7)$ as distribuições iniciais no tempo $t=0$.

É claro que o sistema de edp FIMAN, (0.1) - (0.4), é um sistema misto parabólico-hiperbólico, já que as equações $(0.1)$, (0.3) são do tipo parabólico e a equação escalar (0.4) é do tipo hiperbólico.

\subsection{Algumas questões físicas do sistema FIMAN}

A elegante equação do movimento

$$
\begin{aligned}
& \rho \frac{D}{D t} u=\rho f+\operatorname{div} T \\
& \frac{\partial \rho}{\partial t}+\operatorname{div}(\rho u)=0
\end{aligned}
$$


onde $\frac{D}{D t}=\frac{\partial}{\partial t}+u \cdot \nabla$ denota a derivada material, e cada componente da matriz $T, T_{i j}=T_{i j}(x, t)$ representa fisicamente a $j$-ésima componente da força sobre o elemento de superfície com normal exterior na $i$-ésima direção.

A equação (0.8) foi descoberta por Cauchy em 1828 e junto com (0.9) são válidas para qualquer meio contínuo sem qualquer hipótese na matriz de tensões $T$. Assim, por exemplo, quando os efeitos das tensões tangenciais não são levados em consideração, então um fluido perfeito é, por definição, um material que satisfaz $T=I p$, onde $I$ é a matriz identidade e $p=p(x, t)$ é uma função escalar positiva conhecida como a pressão. Neste caso, (0.8) pode ser escrita como

$$
\rho \frac{D}{D t}=\rho f-\nabla p
$$

Outro caso se apresenta quando a matriz $T$ é relacionada com outras variáveis como cinemáticas e termodinâmicas. Estas relações que determinam o tipo de fluido considerado como fluido viscoso, plástico, elástico, etc., são chamadas de equações constitutivas. Salientamos que é possível obter equações constitutivas no caso de tensões tangenciais consideráveis.

Uma outra equação importante na mecânica de fluidos é a equação do momento angular, isto é,

$$
\frac{\mathrm{d}}{\mathrm{d} t} \int_{\Omega(t)} \rho(r \times u) \mathrm{d} x=\int_{\Omega(t)} \rho(r \times f)+\int_{\partial \Omega(t)} r \times t \mathrm{~d} s
$$

onde $r$ é um vetor posição e $t=\mathbf{n} \cdot T$, com $T=T(x, t)$ é a matriz de tensões e $\mathbf{n}$ é o vetor unitário normal à superfície $\Omega(t)$. Para garantir a conservação do momento angular em fluidos mecânicos é assumido que a matriz $T$ seja simétrica. Embora estas considerações sejam válidas para muitos fluidos comuns, este enfoque, completamente desprovido de considerações de natureza molecular, tem sido criticado por alguns especialistas da mecânica de fluidos, como é o caso de [7], [8] e [11], já que 
para outras situações como em teoria da lubrificação, cristais líqüidos e em meios polarizados a hipótese de simetria da matriz $T$ não é suficiente.

O argumento básico destes especialistas é que os efeitos microscópicos podem gerar efeitos ao nível macroscópico, já que, assim como um campo de fluxos num ponto do fluido é definido como médias locais das velocidades de translações das partículas, então as médias locais das velocidades rotacionais das moléculas, da mesma forma, definem um campo rotacional macroscópicos que será uma representação cinemática do momento angular interno que causa o giro das partículas e estão dinamicamente acopladas à velocidade do fluido por interações de colisão das moléculas em rotação e translação.

De um ponto de vista microscópico, este acoplamento, segundo Condiff e Dolher [9], obedece a uma manifestação de forças moleculares não compensadas. Neste sentido, Born [7] observou que uma exígua linha de fluido que se movimenta terá uma rotação com velocidade $1 / 2$ rot $u$ e encontrará uma resistência devido a rotações internas quando estas não estiverem sincronizadas. Born sugeriu que esta resistência é uma função de rotu-2w, onde $w$ é o campo de rotações, e que tensões assimétricas é a resistência apropriada.

Para obter as equações $(0.1)$ - (0.4), consideramos o cálculo do momento angular total num volume de fluido ao redor de uma origem escolhida. Temos

$$
\begin{aligned}
\frac{D}{D t} \int_{\Omega(t)} \rho M \mathrm{~d} x & =\int_{\Omega(t)} \rho(g+r \times f) \mathrm{d} x+\int_{\partial \Omega(t)} \mathbf{n} \cdot(c+r \times T) \mathrm{d} s \\
& =\int_{\Omega(t)}\left(\rho g+\rho r \times f+\operatorname{div} C+r \times \operatorname{div} r+T_{x}\right) \mathrm{d} x
\end{aligned}
$$

onde $M$ denota o momento angular total (por unidade de massa do fluido), $g$ é um torque (de massa) por unidade de massa que provém de influências internas, $C$ é uma matriz de acoplamento de tensões, $n \cdot T$ representa tensões normais e finalmente $T_{x}=\left(T_{23}-T_{32}, T_{31}-T_{13}, T_{12}-T_{21}\right)$. 
O momento angular total $M$ pode ser decomposto como a soma do momento angular linear $\rho r \times u$ e do momento angular interno $\rho \ell$, proveniente de contribuições de rotações moleculares.

Escrevendo a equação (0.11) na forma diferencial, obtém-se

$$
\rho \frac{D}{D t}(\ell+r \times u)=\rho g+\rho r \times f+\operatorname{div} C+\operatorname{div} T+T_{x}
$$

Fazendo o produto vetorial de $r$ com a equação de Cauchy, temos

$$
\left.\rho\left(r \times \frac{\partial u}{\partial t}\right)=\rho \frac{D}{D t}(r \times u)=\rho r \times f+r \times \operatorname{div} T\right)
$$

e subtraindo as duas últimas equações, obtém-se

$$
\rho \frac{D \ell}{D t}=\rho g+\operatorname{div} C+T_{x}
$$

Assumindo que o momento angular interno por unidade de massa pode ser escrito como um vetor com componentes $\ell_{i}=I_{i k} w_{k}, i=1,2,3$ e, além disso, assumindo a propriedade de isotropia de um fluido, isto é, aqueles fluidos que satisfazem

$$
I_{i k}=I \delta_{i k}
$$

onde $I$ é um escalar chamado de coeficiente de microinércia. Assim, da equação (0.12), podemos escrever

$$
\rho I \frac{\partial w}{\partial t}=\rho g+\operatorname{div} C+T_{x}
$$

Para um fluido micropolar isotrópico com matriz de tensões $T$ escolhida como sendo

$$
T_{i j}=\left(-P+\lambda u_{k, k}\right) \delta_{i j}+\mu\left(u_{i, j}+u_{j, i}\right)+\mu_{r}\left(u_{j, i}-u_{i, j}\right)-2 \mu_{r} \varepsilon_{m, i, j} w_{m}
$$

$\mathrm{e}$

$$
C_{i j}=C_{0} w_{k, k} \delta_{i, j}+C_{d}\left(w_{i, j}+w_{j, i}\right)+C_{a}\left(w_{j, i}-w_{i, j}\right) .
$$


Usamos aqui a notação usual de Einstein. Substituindo $T$ e $C$ na equação de Cauchy e a de momento angular e levando em conta a equação de continuidade, obtém-se o sistema de equações $(0.1)-(0.4)$.

\subsection{Resultados teóricos conhecidos}

Esta seção está dividida em duas partes:

- fluidos micropolares com densidade constante;

- fluidos não-homogêneos assimétricos.

\subsubsection{Fluidos micropolares com densidade constante}

Neste caso, é usual supor que a densidade constante é conhecida (pode ser deteminada em qualquer momento de maneira independente da evolução do fluido). Portanto, a equação (0.4) não acrescenta informação e ela desaparece do modelo.

Este caso foi estudado por Lukaszewicz em uma série de artigos [39], [40], [42], [38]. No artigo [39], ele estuda o problema estacionário associado; em [40], a existência de soluções fracas para o modelo de evolução, e em [42], a existência e unicidade de soluções fortes. Estes trabalhos foram feitos em domínios limitados e a técnica utilizada pelo autor foi linearização em conjunto com um teorema de quase-ponto-fixo. Os resultados obtidos são análogos aos conhecidos para equações de Navier-Stokes clássicas. Ainda em domínios clássicos, porém acoplando uma equação para o campo magnético, Rojas-Medar em [51], [59] estuda a existência de soluções fortes locais utilizando o método de Galerkin espectral. Usando a mesma técnica, Rojas-Medar e Boldrini [65] estudam existência de soluções fracas e o problema de soluções reprodutivas e em Ortega-Torres e Rojas-Medar [58] tratam com soluções globais fortes. 
Analogamente, ao caso das equações de Navier-Stokes, as soluções fracas no problema tridimensional não são necessariamente únicas (de fato, é um dos problemas em aberto clássicos da teoria das equações de Navier-Stokes).

Na teoria das equações de Navier-Stokes, Serrin [77] mostra condições suficientes para a unicidade. Resultados análogos foram estudados no trabalho [56], onde também se estabelece a existência das derivadas fracionárias temporais.

No trabalho [51] é estudado o modelo estacionário com dados de contorno irregulares, isto é, onde não é possível utilizar a teoria de traços. Assim, é introduzida a noção de soluções ultra-fraca ("very weak solutions") e, via argumentos de dualidade e linearização, prova-se a existência de uma solução ultra-fraca. Em [50] é estudada a existência e unicidade de soluções periódicas fortes para uma classe abstrata de equações diferenciais parciais (incluindo as equações micropolares), utilizando novamente a técnica de Galerkin.

Usando um enfoque diferente, o chamado formalismo de "Dubovitskii e Myulitin" de programação matemática em dimensão infinita, no artigo [55] estuda-se problemas de controlabilidade associados às equações micropolares estacionárias.

para certos casos de domínios não-cilíndricos (quer dizer, domínios que mudam com o tempo), foi estudado em [64], [57]. Para domínios exteriores, em [18] estudase o problema estacionário e em [17] o problema de evolução. Neste último caso, na sua abordagem foi utilizada a técnica de imersão de domínios introduzida por Ladyzhenskaya no estudo das equações de Navier-Stokes (veja também Heywood $[30])$.

\subsubsection{Fluidos não-homogêneos assimétricos}

Passamos a comentar agora os trabalhos realizados para o sistema $(0.1)-(0.4)$. Lukaszewicz [49] (veja também [48]) prova a existência de soluções fracas ao mesmo 
nível que os resultados obtidos por Kim [36] para o caso dos fluidos não-homogêneos (isto é, o caso quando não é considerada a equação do momento angular (0.3)).

Para o caso de soluções fortes, tanto local como global, foram estudados nos artigos [10] e [8], usando o método de semi-Galerkin espectral. Por último, a existência e unicidade de soluções fortes foi provada em [14] usando um método iterativo.

Salientamos que todos estes trabalhos foram feitos em domínios limitados e no nosso entender, o resultado principal do Capítulo 2 deste trabalho sobre soluções fortes num domínio não-limitado é novo para o sistema de Edp (0.1) - (0.7).

A Estructura deste trabalho de tese é a seguinte; No Cap 'itulo 1, apresentamos resultados e definições necessários que serã o utilizados nos Cap 'itulos posteriores. No Cap 'itulo 2, obtemos um resultado sobre existência de soluções fortes, local no tempo, sobre um dom 'inio tridimensional não limitado. No cap 'itulo 3 , apresentamos um resultado de existência de soluções fracas reprodutivas, e finalmente, apresentamos um resultado de existência de soluções fracas para uma desigualdade variacional associada ao sistema de equações dos fluidos assimétricos não Homogêneos.

Finalmente, gostaríamos de comentar que neste trabalho adaptamos técnicas de Ladyzhenskaya-Solonnikov [41], Okamoto [54], Simon [78], Salvi [70], Itoh-Tani [31], desenvolvidas para as equações de fluidos não-homogêneos. Os aportes originais deste trabalho são apresentados nos Capítulos 2, 3, 4 e no início de cada capítulo, fazemos comentários pertinentes em relação aos resultados lá obtidos. 


\section{Capítulo 1}

\section{Preliminares}

Esta seção contém todos os conceitos e resultados de Análise Funcional que utilizaremos nos outros capítulos. Como Distribuições, espaços de Sobolev, espaços funcionais com "divergência nula", a valores reais ou a valores num espaço de Banach, etc. Assim como suas propriedades: Imersões contínuas e densas, compacidade, reflexibilidade, separabilidade, dualidade e os diferentes tipos de convergências, forte, fraca * e no sentido das distribuições.

Utilizaremos as notações: $\|\cdot\|$ para as normas, $(\cdot, \cdot)$ para indicar os produtos internos e $\langle\cdot, \cdot\rangle$ para os produtos de dualidade (incluídos os associados a uma distribuição), indicando às vezes, quando for necessário, os espaços considerados como sub-índices para evitar confusões.

\subsection{Espaços de Sobolev com valores reais}

Seja $\Omega \subset \mathbb{R}^{N}$ aberto qualquer, se $f \in L_{\text {loc }}^{1}(\Omega)$, então $f$ determina de forma unívoca uma distribuição (distribuição regular), que denotaremos por $f$ e é definida por

$$
\langle f, \varphi\rangle=\int_{\Omega} f(x) \varphi(x) d x=(f, \varphi) \forall \varphi \in D(\Omega)
$$

onde $D(\Omega)=\left\{\varphi \in C_{0}^{\infty}(\Omega)^{3}\right\}$. Portanto o produto dualidade entre $D^{\prime}(\Omega)$ e $D(\Omega)$ generaliza o produto escalar em $L^{2}(\Omega)$. 
Lembremos que, dada $T \in D(\Omega)$, faz sentido falar de quaisquer derivada de $T$, associadas ao multi-índice $\alpha=\left(\alpha_{1}, \ldots, \alpha_{N}\right)$, no sentido das distribuições. Trata-se de uma nova distribuição $\partial^{\alpha} T$, definida por

$$
\left\langle\partial^{\alpha} T, \varphi\right\rangle=(-1)^{|\alpha|}\left\langle T, \partial^{\alpha} \varphi\right\rangle \quad \forall \varphi \in D(\Omega)
$$

Além disso, se em particular $T$ é uma função regular, $\partial^{\alpha} T$ e a derivada usual de $T: \frac{\partial^{\alpha} T}{\partial x_{1}^{\alpha_{1}} \cdots \partial x_{N}^{\alpha_{N}}}$ coincidem.

Os espaços de Sobolev $W_{p}^{m}(\Omega)$ definidos por

$$
W_{p}^{m}(\Omega)=\left\{v \in L_{p}(\Omega) / \partial^{\alpha} v \in L_{p}(\Omega), \quad \forall|\alpha| \leq m\right\}
$$

são espaços de Banach com normas

$$
\begin{gathered}
\|v\|_{W_{p}^{m}(\Omega)}=\left(\sum_{|\alpha| \leq m} \int_{\Omega}\left|\partial^{\alpha} v\right|^{p} d x\right)^{1 / p}=\left(\sum_{|\alpha| \leq m}\left\|\partial^{\alpha} v\right\|_{L_{p}(\Omega)}^{p}\right)^{1 / p}, \text { se } p<+\infty \\
\|v\|_{W^{m, \infty}(\Omega)}=\sup _{|\alpha| \leq m}\left(\sup _{X \in \Omega} \text { ess }\left|\partial^{\alpha} v(\alpha)\right|\right) \\
=\sup _{|\alpha| \leq m}\left\|\partial^{\alpha} v\right\|_{L^{\infty}(\Omega)} \quad \text { se } p=+\infty
\end{gathered}
$$

Observemos que, os espaços $\stackrel{\circ}{W}_{p}^{m}(\Omega)$ (fecho de $D(\Omega)$ em $W_{p}^{m}(\Omega)$ ) ainda são espaços de Banach pela norma induzida por $W_{p}^{m}(\Omega)$. Pela desigualdade de PoincaréFriedrich, se $\Omega$ estiver limitado pelo menos em uma direção dos eixos coordenados, a seminorma abaixo, é de fato uma norma de $H_{0}^{m}(\Omega)=\stackrel{\circ}{W}_{2}^{m}(\Omega)$, equivalente à norma induzida de $H^{m}(\Omega)=W_{2}^{m}(\Omega)$ :

$$
\|v\|_{H_{0}^{m}(\Omega)}=\left(\sum_{|\alpha|=m}\left\|\partial^{\alpha} v\right\|_{L^{2}(\Omega)}^{2}\right)^{1 / 2} .
$$

No caso particular, para $p=2$, os espaços de Sobolev (denotados por $H^{m}(\Omega)$ e $H_{0}^{m}(\Omega)$ respectivamente) são espaços de Hilbert com os produtos escalares abaixo

$$
\begin{aligned}
(u, v)_{H^{m}(\Omega)} & =\sum_{|\alpha| \leq m} \int_{\Omega} \partial^{\alpha} u(x) \partial^{\alpha} v(x) d x=\sum_{|\alpha| \leq m}\left(\partial^{\alpha} u, \partial^{\alpha} v\right) \\
(u, v)_{H_{0}^{m}(\Omega)} & =\sum_{|\alpha|=m}\left(\partial^{\alpha} u, \partial^{\alpha} v\right) .
\end{aligned}
$$


Se $\Omega$ é limitado, a aplicação linear $\left.v \mapsto v\right|_{\partial \Omega}$, definida de $C^{1}(\bar{\Omega})$ em $L^{2}(\Omega)$, é contínua quando o espaço vetorial $C^{1}(\bar{\Omega})$ é considerado com a norma de $H^{1}(\Omega)$. Em conseqüência, possui uma única prolongação contínua a todo $H^{1}(\Omega)$, chamada de aplicação traço e denotada usualmente por $\gamma$. Logo, temos

$$
\gamma \in \mathcal{L}\left(H^{1}(\Omega), L^{2}(\partial \Omega)\right) \quad \text { e } \quad \gamma v=\left.v\right|_{\partial \Omega}
$$

para $v$ "regular".

Isto permite falar dos "valores fronteiras" de $v$, para cada $v \in H^{1}(\Omega)$. Geralmente escreve-se $\left.v\right|_{\partial \Omega}$ em lugar de $\gamma v$, ainda quando $v$ não é regular. Para $\Omega \subset \mathbb{R}^{N}$, limitado e de fronteira Lipschitziana, temos que o núcleo da aplicação traço coincide com $H_{0}^{1}(\Omega)$. No caso de funções com valores em $\mathbb{R}^{N}(N \in \mathbb{R})$, o número $N$ de componentes é super-indicado. Por exemplo,

$$
v \in\left(W_{p}^{m}(\Omega)\right)^{N} \Leftrightarrow v=\left(v_{1}, v_{2}, \cdots, v_{N}\right) \quad \text { e } \quad v_{i} \in W_{p}^{m}(\Omega), 1 \leq i \leq N .
$$

De fato, $\left(W_{p}^{m}(\Omega)\right)^{N}$ ainda é um espaço de Banach para a norma produto usual. O produto escalar de $\left(H^{m}(\Omega)^{N}\right)$ e de $\left(H_{0}^{m}(\Omega)^{N}\right)$, tem naturalmente a mesma definição (1.1), porém trocamos $\partial^{\alpha} u \partial^{\alpha} v$ por $\partial^{\alpha} u \cdot \partial^{\alpha} v$ (produto escalar euclideano dos vetores $\partial^{\alpha} u$ e $\left.\partial^{\alpha} v\right)$

Pelo Teorema de Riesz, identificaremos o espaço de Hilbert $L^{2}(\Omega)$ com seu dual, considerando, além disso que, para os outros $L^{p}(\Omega)$, o produto dualidade generaliza o produto escalar de $L^{2}(\Omega)$. Logo, fará sentido escrever $\left(L^{p}(\Omega)\right)^{\prime}=L^{p^{\prime}}(\Omega)$ onde $1 \leq p<+\infty$, com $p^{\prime}$ expoente conjugado de $p$ e

$$
\langle v, u\rangle_{L^{p^{\prime}}(\Omega) L^{p}(\Omega)}=\int_{\Omega} v(x) u(x) d x \forall v \in L^{p^{\prime}}(\Omega), \forall v \in L^{p}(\Omega) .
$$

Pelo teorema de Hanh-Banach, $W^{-m, p}(\Omega)=\left(\stackrel{\circ}{W}_{p}^{m}(\Omega)\right)^{\prime}$ dual de $\stackrel{\circ}{W}_{p}^{m}(\Omega)$ pode ser identificado ao espaço das distribuições

$$
W^{-m, p^{\prime}}(\Omega)=\left\{S: S \in \mathcal{D}^{\prime}(\Omega), S=\sum_{|\alpha| \leq m} \partial^{\alpha} v_{\alpha}, \quad v_{\alpha} \in L^{p^{\prime}}(\Omega)\right\},
$$


com o produto dualidade seguinte

$$
\langle S, \varphi\rangle=\sum_{|\alpha| \leq m}(-1)^{|\alpha|}\left(v_{\alpha}, \partial^{\alpha} \varphi\right)_{L^{p^{\prime}}(\Omega) L^{p}(\Omega)}
$$

Isto é, são equivalentes

(a) $T \in W^{-m, p}(\Omega)$;

(b) $T$ é o prolongamento a todo $\stackrel{\circ}{W}_{p}^{m}(\Omega)$ de uma forma linear contínua $S: \mathcal{D}(\Omega) \mapsto$ $R$ que verifica:

$$
\exists C>0 \text { tal que } \mid\langle S, \varphi>| \leq C\|\varphi\|_{W^{m, p}(\Omega)} \forall \varphi \in \mathcal{D}(\Omega)
$$

(c) T é a prolongação a todo $\stackrel{\circ}{W}_{p}^{m}(\Omega)$ de uma distribuição $S$ sobre $\Omega$ que está em $W^{-m, p^{\prime}}(\Omega)$.

Teorema 1.1 [Imersões contínuas e compactas de Sobolev]. Seja $\Omega$ aberto limitado de $R^{N}$ com fronteira Lipschitziana. As seguintes imersões são contínuas e densas:

$$
W_{p}^{1}(\Omega) \hookrightarrow L^{p \star}(\Omega) \text { com } \begin{cases}1 / p^{\star}=1 / p-1 / N & \text { se } p<N ; \\ p_{\star} \in[1,=\infty) & \text { se } p=N ; \\ p_{\star}=+\infty & \text { se } p>N ;\end{cases}
$$

e quando $1 \leq s<p_{\star}$ a imersão é compacta em $L^{s}(\Omega)$.

Em geral, as seguintes imersões são contínuas e densas

$$
W_{p}^{m}(\Omega) \hookrightarrow W_{q}^{n}(\Omega) \quad c o m \begin{cases}\frac{1}{q}=\frac{1}{p}-\frac{(m-n)}{N} & \text { se }(m-n) p<N ; \\ q \in[1,+\infty) & \text { se }(m-n) p=N ; \\ q=+\infty & \text { se }(m-n) p>N\end{cases}
$$

( $n$ inteiro, $0 \leq n \leq m, q \geq 1$ ) e a imersão é compacta em $W^{n, s}(\Omega)$ para cada $s<q$ $(s \geq 1)$. Além disso, temos as seguintes imersões compactas: $W_{p}^{m}(\Omega) \hookrightarrow C^{k}(\Omega)$ se $m p>N$ e $k$ é o maior inteiro tal que $0 \leq k<m-N / p$.

Corolário 1.2 Nas condiçôes do Teorema 1.1, e $p, s \neq+\infty$, então temos as seguintes imersões contínuas e densas (compactas, resp.)

$$
W^{-n, q^{\prime}}(\Omega) \hookrightarrow W^{-m, p^{\prime}}\left(\operatorname{resp} . W^{-n, s}(\Omega) \hookrightarrow W^{-m, p \prime}(\Omega)\right)
$$


O próximo teorema é útil no caso de domínios não-limitados. A nossa referência é Ladyzhenskaya [39], pag. 34.

Teorema 1.3 Para qualquer $u \in W_{p}^{1}(\Omega), p \geq 1$, onde $\Omega$ é não necessariamente limitado no $\mathbb{R}^{N}, N \geq 2$, temos que para todo $r \geq 1$

$$
\|u\|_{L^{q}(\Omega)} \leq \beta\|\nabla u\|_{L^{p}(\Omega)}^{\alpha}\|u\|_{L^{r}(\Omega)}^{1-\alpha}
$$

com

$$
\alpha=\left(\frac{1}{r}-\frac{1}{q}\right)\left(\frac{1}{r}-\frac{1}{\bar{p}}\right)^{-1}, \quad \bar{p}=\frac{N p}{N-p}
$$

onde

(i) para $p<N$, temos que, se $r \leq \bar{p}$, então $q \in[r, \bar{p}]$ ou se $r \geq \bar{p}$, então $q \in[\bar{p}, r]$ e $\beta=\left(\frac{(N-1)}{N} \bar{p}\right)^{\alpha}$. Quando $q$ varia entre $r$ e $\bar{p}, \alpha$ varia entre 0 e 1 , incluindo os extremos; se $r=q=\bar{p}, \alpha$ pode ser escolhido arbitrariamente entre $[0,1]$;

(ii) para $p \geq N$, q é um número arbitrário em $[r, \infty) e$

$$
\beta=\max \left\{\left(\frac{N-1}{N}\right) q, 1+\frac{p-1}{p} \cdot \gamma\right\}^{\alpha}
$$

quando q varia de $r$ até $\infty, \alpha$ varia de 0 até $N p /[p N+r(p-N)]$ sem considerar os extremos;

(iii) se $p>N$ a desigualdade ainda vale para $q=\infty \operatorname{com} \beta<\infty$, onde

$$
\alpha=\frac{N p}{N p+r(p-N)} .
$$

\subsection{Espaços de Sobolev com valores num espaço de Banach}

Lembraremos muito brevemente as propriedades dos espaços de funções e distribuições na variável temporal $t \in(0, T)$ a valores num espaço de Banach. 
Seja $B$ um espaço de Banach e $\|\cdot\|_{B}$ sua norma, definimos $L^{p}(0, T, B)$ como sendo

$$
\begin{aligned}
L^{p}(0, T, B)= & \{f:[0, T] \rightarrow B \text { mensuráveis e tal que a função } \\
& \left.t \in[0, T] \mapsto\|f(t)\|_{B} \text { (def. qtp) é } p \text { - somável }\right\}
\end{aligned}
$$

Definimos a norma

$$
\begin{aligned}
\|f\|_{L^{p}(0, T ; B)} & =\left[\int_{0}^{T}\|f(t)\|_{B}^{p} \mathrm{~d} t\right]^{1 / p} \text { se } p<+\infty \\
\|f\|_{L^{\infty}(0, T ; B)} & =\sup _{t \in(0, T)}\|f(t)\|_{B} \text { se } p=+\infty .
\end{aligned}
$$

Estes espaços possuem muitas das propriedades dos espaços $L^{p}(\Omega)$. Se $B$ é reflexivo (resp. separável) e $1 \leq p<\infty$ (resp. $s<p<\infty)$, então $L^{p}(0, T ; B)$ é ainda reflexivo (resp. separável). Se

$D(0, T ; B)=\left\{f:[0, T] \mapsto B\right.$ de classe $C^{\infty}$ e de suporte compacto contido em $\left.(0, T)\right\}$ temos que $\mathcal{D}(0, T ; B)$ é denso no $L^{p}(0, T ; B)$ se $1 \leq p<+\infty$.

Quando $p=2$ e $B=H$ é um espaço de Hilbert com produto escalar $(\cdot, \cdot)_{H}$, então $L^{2}(0, T ; H)$ é ainda um espaço de Hilbert com produto escalar:

$$
(f, g)_{L^{2}(0, T ; H)}=\int_{0}^{T}(f(t), g(t))_{H} \mathrm{~d} t
$$

O espaço vetorial $L_{\text {loc }}^{p}(0, T ; B)$ possui estrutura de espaço de Fréchet (localmente convexo, metrizável e completo) para a família de seminormas $\left\{P_{n}^{p}(v)\right\}_{n \geq 1}$, onde

$$
P_{n}^{p}(v)=\|v\|_{L_{p}\left(\mathbb{K}_{n} ; B\right)} \forall v \in L_{\mathrm{loc}}^{p}(0, T ; B), \forall n \geq 1
$$

e $\left\{\mathbb{K}_{n}\right\}$ é uma seqüência crescente de compactos tal que sua união é todo $(0, T)$. Se $f \in L_{\text {loc }}^{1}(0, T ; B)$, então $f$ define de forma unívoca uma distribuição em $\mathcal{D}^{\prime}(0, T ; B)$ (chamada distribuição regular), que ainda denotamos por $f$, definida como

$$
\langle f, \varphi\rangle=\int_{0}^{T} f(t) \varphi(t) \mathrm{d} t, \forall \varphi \in \mathcal{D}(0, T) .
$$


O símbolo $\langle\cdot, \cdot\rangle$ denota a ação de $f$, uma aplicação linear contínua de $\mathcal{D}(0, T)$ em $B$, sobre $\varphi \in \mathcal{D}(0, T)$.

Se pusermos $Q_{T}=\Omega \times[0, T], T>0$ e $\Omega$ um domínio em $\mathbb{R}^{3}$ não necessariamente limitado, definimos outros tipos de espaços que usaremos posteriormente no Capítulo 2 deste trabalho. São os seguintes:

$$
W_{p}^{2,1}\left(Q_{T}\right)=\left\{v \in L_{p}\left(Q_{T}\right) \mid\|v\|_{W_{P}^{2,1}\left(Q_{T}\right)}=\left\|v_{t}\right\|_{L_{P}\left(Q_{t}\right)}+\sum_{|\alpha| \leq 2}\left\|D_{x}^{\alpha} u\right\|_{L_{p}\left(Q_{T}\right)}<\infty\right\}
$$

onde $D_{x}^{\alpha}=\left(\frac{\partial}{\partial x_{1}}\right)^{\alpha_{1}}\left(\frac{\partial}{\partial x_{2}}\right)^{\alpha_{2}}\left(\frac{\partial}{\partial x_{3}}\right)^{\alpha_{3}}$.

Se $v \in W_{p}^{2,1}\left(Q_{T}\right)$ e $p>3$, para $t \in[0, T]$ fixo, o valor de $v(x, t)$ pertence ao espaço de Slobodetskii-Besov, $W_{p}^{2-2 / p}(\Omega)$ definido por

$$
W_{p}^{2-2 / p}(\Omega)=\left\{v \in L_{p}\left(Q_{T}\right) \mid\|v\|_{W_{p}^{2-2 / p}(\Omega)}<+\infty\right\}
$$

onde

$$
\|v\|_{W_{p}^{2-2 / p}(\Omega)}=\left(\sum_{|\alpha| \leq 1}\left\|D_{x}^{\alpha} u\right\|_{L_{p}(\Omega)}^{p}+\sum_{|\alpha|=1} \int_{\Omega \times \Omega} \frac{\left|D_{x}^{\alpha} u(x)-D_{y}^{\alpha} u(y)\right|^{p}}{|x-y|^{1+p}} \mathrm{~d} x \mathrm{~d} y\right)
$$

O espaço $W_{p}^{2,1}\left(Q_{t}\right)$ é o espaço das distribuições $v \in L^{p}\left(0, T ; W_{p}^{2}(\Omega)^{3}\right)$ tal que $v_{t} \in$ $L^{q}\left(Q_{T}\right)^{3}$.

Faremos uso freqüente da desigualdade abaixo, devida a Solonnikov [83]. Para todo $v \in W_{p}^{2,1}\left(Q_{T}\right)$ e para todo $t, 0 \leq t \leq T$, temos

$$
\|v(t)\|_{W_{p}^{2-2 / p}(\Omega)^{3}} \leq\|v(\cdot, 0)\|_{W_{p}^{2-2 / p}(\Omega)^{3}}+c\|v\|_{W_{p}^{2,1}\left(Q_{T}\right)}
$$

onde a constante $c$ independe de $t$.

Seja $\Omega$ aberto de $\mathbb{R}^{N}$, definimos o espaço vetorial $C^{m}(\bar{\Omega})$ de todas as funções $\varphi \in$ $C^{m}(\Omega)$ tal que $D^{\alpha} \varphi$ é limitada e uniformemente contínua sobre $\Omega$ para $0 \leq|\alpha| \leq m$. Lembremos ainda que $C^{m}(\bar{\Omega})$ é um espaço de Banach com a norma abaixo:

$$
\|\varphi\|_{m}=\|\varphi\|_{C^{m}(\bar{\Omega})}=\max _{0 \leq|\alpha| \leq m} \sup _{x \in \Omega}\left|D^{\alpha} \varphi(x)\right|
$$


Se $0<\lambda \leq 1$, definimos $C^{m, \lambda}(\bar{\Omega})$ como o subespaço de $C^{m}(\bar{\Omega})$ que consiste de todas as funções $\varphi$ tal que, para $0 \leq|\alpha| \leq m, D^{\alpha} \varphi$ satisfaz em $\Omega$ a condição de Hölder com expoente $\lambda$, isto é, existe uma constante $C$ tal que

$$
\left|D^{\alpha} \varphi(x)-D^{\alpha} \varphi(y)\right| \leq C|x-y|^{\lambda}, x, y \in \Omega \text {. }
$$

O espaço $C^{m, \lambda}(\bar{\Omega})$ é um espaço de Banach com a norma

$$
\|\varphi\|_{m, \lambda}=\|\varphi\|_{m}+\max _{\substack{x, y \in \Omega \\ x \neq y}} \frac{\left|D^{\alpha} \varphi(y)-D^{\alpha} \varphi(x)\right|}{|x-y|^{\lambda}} .
$$

No caso $Q_{T}=\Omega \times(0, T)$, definimos para $0 \leq \alpha \leq 1,0 \leq \beta \leq 1$, o espaço $C^{\alpha, \beta}\left(\bar{Q}_{T}\right)$ como o espaço das funções $\varphi$ definidas sobre $\bar{Q}_{T}$ que são Hölder contínuas com expoente $\alpha$ e $\beta$ em relação a $x$ e $t$, respectivamente. Definimos sua norma por

$$
\|\varphi\|_{C^{\alpha, \beta}(Q, T)}=\sup _{\bar{Q}_{T}} \varphi(x, t)+[u]_{C^{\alpha, \beta}\left(\bar{Q}_{T}\right)}
$$

onde

$$
[u]_{C^{\alpha, \beta}\left(\bar{Q}_{T}\right)}=\sup _{\substack{(x, t),(y, s) \in \bar{Q}_{T} \\ x \neq y, t \neq s}} \frac{|\varphi(x, t)-\varphi(y, s)|}{|x-y|^{\alpha}+|t-s|^{\beta}} .
$$

Para mais detalhes destes espaços, veja Adams [1], Ladyzhenskaya [38] e Kufner et al. [37].

\subsection{Produtos contínuos entre espaços de Sobolev reais em dimensão 3}

Lema 1.4 Seja $\Omega$ nas condições do Teorema 1.1.

(i) Para $1 \leq s \leq r \leq+\infty$, a aplicação produto de $W^{1, r}(\Omega) \times W^{1, s}(\Omega)$ em $W^{1, t}(\Omega)$, com $\frac{1}{t}=\frac{1}{\gamma^{*}}+\frac{1}{s}\left(r^{*}\right.$ dado pelo Teorema 1.1) está bem definida e é contínua se $t \geq 1$. 
(ii) Para $1 \leq \gamma \leq+\infty$ e $1<s \leq+\infty$, a aplicação produto $W^{1, \gamma}(\Omega) \times W^{-1, s}(\Omega)$ em $W^{-1, t}(\Omega)$, definida por

$$
\langle\mu u, v\rangle_{W^{-1, t}(\Omega)}=\langle\mu, u v\rangle_{W^{-1, s}(\Omega)}
$$

$\forall \mu \in W^{-1, s}(\Omega), \forall u \in W^{1, r}(\Omega) e \forall v \in W_{0}^{1, t^{\prime}}$, onde $t^{\prime} e^{\prime} \frac{1}{t}+\frac{1}{t^{\prime}}=1$, está bem definida e é contínua se $t>1$.

\subsection{Resultado de compacidade}

Passamos a definir agora os espaços de Nikolskii. Seja $B$ um espaço de de Banach e $f:(0, T) \mapsto B$ e $h>0$, definimos a função $\tau_{h}$

$$
\tau_{h} f:(-h, T-h) \mapsto B
$$

definida por

$$
\left(\tau_{h} f\right)(t)=f(t+h), \quad \forall t \in(-h, T-h)
$$

Definimos o espaço de Nikolskii da ordem $s(0<s \leq 1)$ e expoente $q(1 \leq q \leq$ $+\infty)$ por

$$
N^{s, q}(0, T ; B)=\left\{f: f \in L^{q}(0, T ; B),\left\|\tau_{h} f-f\right\|_{L^{q}(0, T-h ; B)} \leq C h^{s}, \forall h \in(0, T)\right\}
$$

O espaço $N^{s, q}(0, T ; B)$ possui uma estrutura de espaço vetorial para as operações usuais e com a norma

$$
\|f\|_{N^{s, q}(0, T ; B)}=\|f\|_{L^{q}(0, T ; B)}+\sup _{0<h<T}\left[h^{-s}\left\|\tau_{n} f-f\right\|_{L^{q}(0, T-h ; B)}\right]
$$

é um espaço de Banach. Observe-se que $N^{s, \infty}(0, T ; B)$ é o espaço das funções Höldercontínuas (ou Holderianas) de expoente $s$ e a valores em $B$.

Lema 1.5 (Simon [80]) Sejam $X \hookrightarrow B \hookrightarrow Y$ espaços de Banach com imersões contínuas e densas, a primeira delas compacta. Então as seguintes imersões são compactas: 
(i) $L^{q}(0, T ; X) \cap\left\{\phi ; \frac{\partial \phi}{\partial t} \in L^{1}(0, T ; Y)\right\} \hookrightarrow L^{2}(0, T ; B)$ se $1 \leq q \leq+\infty$;

(ii) $L^{\infty}(0, T ; X) \cap\left\{\phi: \frac{\partial \phi}{\partial t} \in L^{r}(0, T ; Y)\right\} \hookrightarrow C([0, T] ; B)$ se $1<r \leq+\infty$;

(iii) $L^{q}(0, T ; X) \cap N^{s, q}(0, T ; Y) \hookrightarrow L^{q}(0, T ; B)$ se $0<s \leq 1,1 \leq q \leq+\infty$.

\subsection{Espaços de funções com "divergente nulo"}

Seja $\Omega \subset \mathbb{R}^{3}$ um domínio limitado com fronteira $\partial \Omega$ Lipschitziana e definamos

$$
\begin{aligned}
\mathcal{V} & =\left\{\varphi: \varphi \in \mathcal{D}(\Omega)^{3}, \operatorname{div} \varphi=0 \text { em } \Omega\right\} ; \\
H & =\text { Fecho de } \mathcal{V} \text { em } L^{2}(\Omega)^{3} \\
V & =\text { Fecho de } \mathcal{V} \text { em } H^{1}(\Omega)^{3} .
\end{aligned}
$$

Como $\partial \Omega$ é Lipschitziana, podemos caracterizar os espaços $V$ e $H$ como

$$
\begin{aligned}
& H=\left\{v \in L^{2}(\Omega)^{3} ; \operatorname{div} v=0 \text { em } \Omega,\left.(v \cdot \overrightarrow{\mathrm{n}})\right|_{\Gamma}=0 \text { sobre } \partial \Omega\right\} \\
& V=\left\{v \in H^{1}(\Omega)^{3}: \operatorname{div} v=0 \text { em } \Omega,\left.v\right|_{\Gamma}=0 \text { sobre } \partial \Omega\right\}
\end{aligned}
$$

onde $\left.v\right|_{\Gamma}$ e $\left.v \cdot \overrightarrow{\mathrm{n}}\right|_{\Gamma}$ e o traço de $v$ e traço normal de $v$ sobre $\partial v .\left.v\right|_{\Gamma}$ faz sentido no $L^{2}(\Gamma)$ se $v \in V$ e $(v \cdot \overrightarrow{\mathbf{n}})_{\Gamma}$ no $H^{-1 / 2}(\Gamma)$ se $v \in H$.

Lema 1.6 [De Rham]. Seja $\Omega \subset \mathbb{R}^{3}$ aberto limitado, de fronteira $\Gamma=\partial \Omega$ suficientemente regular. Se $w \in H^{-1}(\Omega)^{3}$ tal que $\langle w, v\rangle_{\left(H^{-1}\right)^{3},\left(H_{0}^{1}\right)^{3}}=0$ para cada $v \in \mathcal{V}$, então

$$
\exists q \in L^{2}(\Omega) \text { tal que } w=\nabla q
$$

( $q$ é única a menos de uma constante aditiva se $\Omega$ for conexo). Além disso, para cada função w nestas condições, é possível escolher q tal que a aplicaçào $w \mapsto L_{w}=q$ seja linear e contínua par as normas de $H^{-1}(\Omega)^{3}$ e $L^{2}(\Omega)$. 
Teorema 1.7 [Teorema do ponto fixo de Schauder]. Sejam X espaço de Banach, $S \subset X$ um conjunto convexo, fechado e limitado de $X$ e $\phi: S \rightarrow S$ uma aplicação compacta, quer dizer, contínua tal que $\phi(S)$ é relativamente compacto. Então $\phi$ tem pelo menos um ponto fixo em $S$.

Utilizaremos o Terema do ponto fixo de Schauder no Capítulo 3.

Para maiores comentários e demonstrações dos resultados das seções anteriores, sugerimos Simon [80], [81] e as referências ali citadas. 


\section{Capítulo 2}

\section{Existência e unicidade de soluções fortes num domínio não-limitado}

\subsection{Introdução e resultado principal}

Nosso objetivo neste capítulo é o estudo de soluções fortes para o sistema (0.1) (0.7). De maneira mais precisa, vamos provar o Teorema 2.1 (veja na página 22) sobre um domínio não limitado, no contexto dos espaços $L^{p}(\Omega)$ com $p>N=3$.

Distinguimos basicamente três tipos de soluções: fracas, fortes e clássicas, dependendo de sua regularidade. A diferença está no sentido em que as equações (0.1), (0.3) são satisfeitas. Um sentido distribucional (solução fraca), ou pontual q.t.p (solução forte). As soluções fortes, embora não sejam soluções clássicas, são importantes, já que quando existem são únicas na classe das soluções fracas. Um outro ponto importante deste tipo de soluções está ligado diretamente à equação de

continuidade (0.4). É conhecido que (0.4) juntamente com a condição inicial (0.7) e fixada a velocidade $u$, é um problema de transporte (hiperbólico) que pode ser resolvido sob determinadas condições de regularidade sobre os dados, pelo método das características ([15]). No entanto, determinadas propriedades de regularidade de $\rho_{0}$ e $u$, assim como de suas derivadas, são necessárias para obter existência e unicidade da solução para a equação do transporte (veja Ladyzhenskaya-Solonnikov [41], Oka- 
moto [54]). Neste ponto, é necessário resolver um sistema de equações diferenciais ordinárias das "curvas características" usando o método das aproximações sucessivas. Por exemplo, hipóteses de regularidade que levam á existência e a unicidade de soluçẽs de $(0.4),(0.7)$ são as seguintes:

$$
\nabla u \in L^{1}\left(0, T ; L^{\infty}(\Omega)\right) \quad \text { e } \quad \rho_{0} \in W_{\infty}^{1}(\Omega)
$$

No caso das soluções fracas do sistema $(0.1)$ - (0.7), as hipóteses de regularidade requeridas sobre $u$ e $\rho_{0}$ são mais fracas. Isto explica a necessidade de introduzir soluções fortes com maiores hipótese de regularidade. Neste trabalho as estimativas

$$
\|\nabla u\|_{L_{\infty}(\Omega)},\|\nabla w\|_{L_{\infty}(\Omega)},\|\rho\|_{L_{\infty}(\Omega)},\|\nabla \rho\|_{L_{\infty}(\Omega)}
$$

foram essenciais em vários pontos deste trabalho, como por exemplo na prova de unicidade do resultado principal.

O problema de existência e unicidade soluções fortes no caso que o domínio é limitado foi abordado em Boldrini-Rojas-Medar [10], obtendo existência e unicidade de soluções fortes locais e globais no contexto de $L^{2}(\Omega)$ usando como ingrediente básico o método de semi-Galerkin espectral, visando aplicações numéricas.

No caso $N=3$ a existência global é obtida considerando dados iniciais pequenos, mais especificamente que $\left\|u_{0}\right\|_{V}$ e $\left\|w_{0}\right\|_{\left(H_{0}^{1}(\Omega)\right)^{3}},\|f\|_{L^{\infty}\left(0, \infty ;\left(H^{1}(\Omega)\right)^{3}\right)}$ e $\|g\|_{L^{\infty}\left(0, \infty ;\left(H^{1}(\Omega)\right)^{3}\right)}$ sejam suficientemente pequenos (observemos que condições análogas são assumidas para as equações de Navier-Stokes clássicas, veja Heywood [30]) e que quando $N=2$ obtém-se existência global sem a hipótese mencionada anteriormente.

Resultados análogos foram obtidos em C. Conca et al. [14], Rojas-Medar [56] usando um procedimento iterativo. Este último obteve soluções fortes locais e Ortega-Torres-Rojas-Medar [68] obtiveram soluções fortes globais. No entanto, quando $\Omega \subset \mathbb{R}^{3}$ é um domínio não limitado, o problema não tem sido estudado. Os 
problemas em domínios não limitados são podem ser difíceis, pois neles já não temos a validade de imersões compactas entre espaços que são muito úteis nos domínios limitados. Assim, as técnicas utilizadas nos domínios não-limitados devem ser mais depuradas. Para isto, Várias técnicas foram desenvolvidas como semigrupos, integrais singulares, espaços de Sobolev com pesos ou generalizações. Uma técnica interessante é a imersão de domínio, originalmente introduzida por Ladyzhenskaya [38] para o estudo das equações de Navier-Stokes e exposta de maneira interessante emHeywood [30]. Estas técnicas poderiam ser usadas para estudar o sistema (0.1) - (0.7): Porém preferimos usar os chamados espaços de Slobodekstskii-Besov em conjunto com linearização e o método das aproximações sucessivas para realizar a análise.

Este procedimento foi originalmente utilizado por Ladyzhenskaya [38] e LadyzhenskayaSolonnikov [41], para o caso das equações de Navier-Stokes clássicas e não-homogêneas e usadas posteriormente usado por Itoh-Tani em [31].

Observemos que os Lemas 2.2 e 2.4 enunciados posteriormente são devidos a Itoh-Tani [31];o Lema 2.3 devido a Ladyzhenskaya-Solonnikov [41]; e o Lema 2.5 é novo para domínios não limitados. Para sua demonstração utilizamos o método do regularizador que, para mais detalhes, pode ser encontrado nas referências [40] e [83].

A seguir enunciamos o principal resultado deste capítulo.

Teorema 2.1 Suponhamos $p>3$ e as hipóteses seguintes:

$$
\begin{aligned}
& \left.0<m \leq \rho_{(} x\right) \leq M<+\infty, \\
& \rho_{0}(x) \in C(\bar{\Omega}), \quad \nabla \rho_{0} \in W_{p}^{1}(\Omega), \\
& u_{0}(x) \in W_{p}^{2-2 / p}(\Omega),\left.\quad u_{0}\right|_{S}=0, \quad \operatorname{div}\left(u_{0}\right)=0, \\
& w_{0}(x) \in W_{p}^{2-2 / p}(\Omega),\left.\quad w_{0}\right|_{S}=0, \quad f, g \in L_{p}\left(Q_{T}\right) .
\end{aligned}
$$


Então existe um tempo $T_{1} \in(0, T]$ tal que o problema (0.1) - (0.7) possui uma única solução $(u, w, \rho, p)$ que satisfaz

$$
\begin{aligned}
& \rho \in C\left(\bar{Q}_{T_{1}}\right), \nabla \rho \in C\left(\left[0, T_{1}\right] ; W_{p}^{1}(\Omega)\right) \\
& 0<m \leq \rho(x, t) \leq M<+\infty \\
& u, w \in W_{p}^{2,1}\left(Q_{T_{1}}\right) \\
& \nabla p \in L_{p}\left(Q_{T_{1}}\right)
\end{aligned}
$$

Antes de enunciar os lemas auxiliares para demonstrar o Teorema 2.1, faremos algumas observações em relação ao resultado. As equações (0.1) - (0.4) são satisfeitas no sentido q.t.p (em quase todo ponto) sobre $\Omega \times\left(0, T_{1}\right)$. As condições de contorno para $u(x, t), w(x, t)$ são satisfeitas no sentido do traços e q.t.p sobre $(0, T)$. Finalmente, as condições iniciais para $u$ e $w$ são satisfeitas em $W_{p}^{2-2 / p}(\Omega)$ e a condição inicial para a densidade $\rho(x, 0)=\rho_{0}(x)$ em $C(\bar{\Omega})$. Observa-se ainda que, devido a que $W_{p}^{1}(\Omega) \subset C^{0, \lambda}(\Omega)$ com imersão contínua e $0<\lambda<1-3 / p$, temos que $\nabla \rho(x, t) \in C\left(\left[0, T_{1}\right] ; C^{0, \lambda}(\Omega)\right)$. Além disso, se $\Omega$ for limitado com fronteira $C^{2}$, as soluções $u, w \in W_{p}^{2,1}\left(Q_{T_{1}}\right)$ possuem uma regularidade maior, já que neste caso existe um $\lambda=\lambda_{p}$ (que depende de $p$ ) com $0<\lambda_{p}<1$ tal que $W_{p}^{2,1}\left(Q_{T_{1}}\right) \hookrightarrow$ $L^{p}\left(0, T_{1} ; C^{1, \lambda}(\bar{\Omega})^{3}\right) \cap C\left([0, T] ; C(\bar{\Omega})^{3}\right)$, com imersão compacta (veja J. Lemoine [42]).

Agora passamos a enunciar os lemas necessários para realizar a prova do Teorema 2.1 .

\subsection{Resultados auxiliares}

Lema 2.2 (Itoh-Tani [31]) Sejam $\rho(x, t) \in C^{\alpha, \beta}\left(Q_{T}\right)$, onde $\alpha, \beta \in(0,1)$ e $0<m \leq$ $\rho_{0}(x) \leq M<+\infty$. Então, para qualquer função $F \in L_{p}\left(Q_{T}\right), u_{0}(x) \in W_{p}^{2-2 / p}(\Omega)$, $\left.u_{0}(x)\right|_{\sum_{T}}=0$ e div $u_{0}(x)=0$, existe uma única solução do sistema de equações 
diferenciais parciais

$$
\begin{aligned}
& \rho \frac{\partial u}{\partial t}-\left(\mu+\mu_{\gamma}\right) \Delta u+\nabla p=F \\
& \operatorname{div} u=0 \\
& \left.u\right|_{\sum_{T}}=0 \\
& u(x, 0)=u_{0}(x)
\end{aligned}
$$

tal que $u \in W_{p}^{2,1}\left(Q_{T}\right)$ e satisfaz em $\Omega$ a seguinte estimativa

$$
\|u\|_{W_{P}^{2,1}\left(Q_{T}\right)}+\|\nabla p\|_{L_{p}\left(Q_{T}\right)} \leq K_{1}\left(\|\rho\|_{C^{\alpha, \beta}\left(Q_{T}\right)}, T\right)\left(\left\|u_{0}\right\|_{W_{p}^{2-2 / p}(\Omega)}+\|F\|_{L_{p}\left(Q_{T}\right)}\right)
$$

onde $K_{1}$ é uma função crescente de $\|\rho\|_{C^{\alpha, \beta}}\left(\bar{Q}_{T}\right)$ e $T$ e depende das constantes $m e$ $M$.

Lema 2.3 (Ladyzhenskaya-Solonnikov [41]) Seja $u$ satisfazendo div $u=0,\left.u\right|_{\Sigma_{T}}=0$ e a seguinte estimativa

$$
\|u\|_{L^{\infty}\left(Q_{T}\right)}+\int_{0}^{T}\|\nabla u(s)\|_{L^{\infty}(\Omega)} \mathrm{d} s<+\infty
$$

Então para qualquer $\rho_{0} \in C^{1}(\bar{\Omega})$ tal que $0<m \leq \rho_{0}(x) \leq M<+\infty$, o problema escalar

$$
\begin{aligned}
& \frac{\partial \rho}{\partial t}+(u \cdot \nabla) \rho=0 \\
& \rho(x, 0)=\rho_{0}(x)
\end{aligned}
$$

possui uma única solução $\rho \in C^{1,1}\left(Q_{T}\right)$ que satisfaz

$$
\begin{aligned}
m & \leq \rho(x, t) \leq M \\
\|\nabla \rho\|_{L^{\infty}\left(Q_{T}\right)} & \leq \sqrt{3}\left\|\nabla \rho_{0}\right\|_{L_{\infty}\left(Q_{T}\right)} \exp \left(\int_{0}^{T}\|\nabla u(s)\|_{L^{\infty}(\Omega)} \mathrm{d} s\right), \\
\left\|\rho_{t}\right\|_{L^{\infty}\left(Q_{T}\right)} & \leq \sqrt{3}\|u\|_{L^{\infty}\left(Q_{T}\right)}\left\|\nabla \rho_{0}\right\|_{L^{\infty}(\Omega)} \exp \left(\int_{0}^{T}\|\nabla u(s)\|_{L^{\infty}(\Omega)} \mathrm{d} s\right) .
\end{aligned}
$$


Além disso, se $\nabla \rho_{0} \in W_{p}^{1}(\Omega), u(x, t) \in L^{1}\left(0, T ; W_{p}^{2}(\Omega)\right)$, então

$$
\frac{\mathrm{d}}{\mathrm{d} t}\|\nabla \rho(t)\|_{W_{p}^{1}(\Omega)} \leq C\|u(t)\|_{W_{p}^{2}(\Omega)}\|\nabla \rho(t)\|_{W_{p}^{1}(\Omega)} .
$$

Lema 2.4 (Itoh-Tani [31]) Seja u como no Lema 2.3. Se $\rho \in C^{1,1}\left(\bar{Q}_{T}\right)$ é tal que

$$
\begin{aligned}
& \frac{\partial \rho}{\partial t}+(u \cdot \nabla) \rho=h \\
& \rho(x, 0)=\rho_{0}(x)
\end{aligned}
$$

com $h \in L^{1}\left(0, T ; L^{\infty}(\Omega)\right)$, então temos

$$
\|\rho(t)\|_{L^{\infty}(\Omega)} \leq \int_{0}^{t}\|h(s)\|_{L^{\infty}(\Omega)} \mathrm{d} s .
$$

Na monografia [82], Solonnikov apresentou o método do Regularizador para tratar sistemas de equações diferenciais parabólicas. A noção de parabolicidade usada em [82] é suficientemente geral para cobrir nosso caso, pois o problema (2.1) abaixo é parabólico no sentido de Petrovski. A prova do próximo lema está baseada na ordem de idéias expostas em Solonnikov [82], Ladyzhenskaya et al. [40].

Lema 2.5 Seja $\rho \in C^{\alpha, \beta}\left(Q_{T}\right), \alpha, \beta \in(0,1)$, tal que $0<m \leq \rho_{0}(x) \leq M$. Para qualquer função $G \in L_{p}\left(Q_{T}\right), p>\left.3 e w_{0}(x) \in W_{p}^{2-2 / p}(\Omega) \operatorname{com} w_{0}\right|_{\sum_{T}}=0$, o problema linear

$$
\begin{aligned}
& \rho \frac{\partial w}{\partial t}-\nu \Delta w-\gamma \nabla \operatorname{div} w+4 \mu_{r} w=G(x, t) \text { em } \Omega \\
& w \equiv 0 \text { sobre } \Sigma_{T} \\
& w(x, 0)=w_{0}(x) \text { em } \Omega
\end{aligned}
$$

possui uma única solução $w \in W_{p}^{2,1}\left(Q_{T}\right)$, satisfazendo as seguintes estimativas para todo $t \leq T$

$$
\begin{aligned}
& \left(\|w\|_{W_{p}^{2,1}\left(Q_{T}\right)}\right)^{p}+\sup _{\tau \leq t}\left(\|w(x, \tau)\|_{W_{p}^{2-2 / p}(\Omega)}\right)^{p} \\
& \leq C \frac{M^{p+1}(t)}{m^{p+1}(t)}\left(1+m^{-1}(t) \sup _{\tau \leq t}\left[\rho(x, \tau]_{\Omega}^{\alpha}\right)^{\frac{2 p}{\alpha}}\left[F^{p}(t)+\|w\|_{L_{p}\left(Q_{t}\right)}^{p}\right]\right.
\end{aligned}
$$


onde

$$
\begin{aligned}
M(t) & =\max \left(1, \max _{Q_{t}} \rho\right) \\
m(t) & =\min \left(1, \min _{Q_{t}} \rho\right) \\
F^{p}(t) & =\|G\|_{L_{p}\left(Q_{t}\right)}^{p}+\left\|w_{0}(x)\right\|_{W_{p}^{2-2 / p}(\Omega)}^{p} .
\end{aligned}
$$

Para a demonstração do Lema 2.5 vamos escrever $w(x, t)=u(x, t)+v(x, t)$, onde as funções $u(x, t)$ e $v(x, t)$ são soluções dos problemas (2.3) e (2.4), respectivamente:

$$
\begin{aligned}
& \frac{\partial u}{\partial t}-\nu \Delta u-\gamma \nabla \operatorname{div}(u)+4 \mu_{r} u=G(x, t), \\
& u \equiv 0 \text { sobre } \Sigma_{T} \\
& u(x, 0)=w_{0}(x) \text { sobre } \Omega
\end{aligned}
$$

com $g(x, t)=(1-\rho) u_{t}$, além disso,sendo

$$
\begin{aligned}
& \rho(x, t) \frac{\partial v}{\partial t}-\nu \Delta v-\gamma \nabla \operatorname{div} v+4 \mu_{r} v=g(x, t), \\
& v \equiv 0 \text { sobre } \Sigma_{T}, \\
& v(x, 0)=0 \text { sobre } \Omega,
\end{aligned}
$$

A equação diferencial parcial (2.3) é parabólica no sentido de Petrovski e portanto podemos aplicar os resultados de [82]. Com efeito, aplicando os resultados do Cap. V, Teorema 5.4, obtém-se uma única solução $u \in W_{p}^{2,1}\left(Q_{T}\right)$ para o problema (2.3) satisfazendo a estimativa para todo $t \leq T$ :

$$
\|u\|_{W_{p}^{2,1}\left(Q_{T}\right)}+\sup _{\tau \leq t}\|u(x, \tau)\|_{W_{P}^{2-2 / p}} \leq C_{1}\left(\|G\|_{L_{p}\left(Q_{T}\right)}+\left\|w_{0}\right\|_{W_{P}^{2-2 / p}(\Omega)}\right) .
$$

Para obter existência e unicidade em $W_{p}^{2,1}\left(Q_{T}\right)$ do problema (2.4) usamos o método do regularizador, que passamos a explicar formalmente. 
O problema (2.4) é considerado num cilindro $Q_{\tau}$ de altura, $\tau$, pequena da seguinte forma (com condições iniciais nulas):

$$
A u=h,
$$

onde $A: V \mapsto H$ é um operador limitado agindo entre dois espaços de Banach. Logo, temos de construir um operador $R: H \mapsto H$ (chamado de Regularizador) que satisfaça as equações

$$
\begin{aligned}
& A R h=h+T h, \forall h \in H, \\
& R A u=u+M u, \forall v \in V,
\end{aligned}
$$

onde $T: H \mapsto H$ e $M: V \mapsto V$ são operadores lineares com norma menor que 1 e portanto os operadores inversos $\left(I_{H}+T\right)^{-1}$ e $\left(I_{V}+M\right)^{-1}$ existem $\left(I_{H}\right.$ e $I_{V}$ denotam as identidades nos respectivos espaços - daqui em diante, omitiremos os sub-índices $H$ e $V)$. Multiplicando pela direita a equação $(2.7)$ por $(I+T)^{-1}$ e pela esquerda por $(I+M)^{-1}$ a equação $(2.8)$, obtém-se

$$
\begin{aligned}
A R(I+T)^{-1} & =I_{H} \\
(I+M)^{-1} R A & =I_{V} .
\end{aligned}
$$

Portanto, o operador $A$ tem inversa à direita e à esquerda e, conseqüentemente, esses operadores são iguais e existe o operador inverso $A^{-1}: H \mapsto V$ limitado,

$$
A^{-1}=R(I+T)^{-1}=(I+M)^{-1},
$$

definido sobre todo o espaço $H$ e sua norma satisfaz

$$
\left\|A^{-1}\right\| \leq\|R\|\left\|(I+T)^{-1}\right\| \leq 2 C
$$

onde a constante $C$ é tal que

$$
\|R h\|_{V} \leq C\|h\|_{H}
$$


Finalmente, deduzimos que a equação (2.6) possui uma única solução para qualquer $h \in H$ e a sua solução satisfaz a desigualdade

$$
\|u\|_{V} \leq\left\|A^{-1}\right\|\|h\|_{H} \leq 2 C\|h\|_{H} .
$$

Feitas estas observações gerais, passamos agora a tratar do problema (2.4). No que segue será útil o sistema de coberturas de $\bar{\Omega}$, ( com fronteira de curvatura limitada )

$C_{\Omega}=\left\{\Omega^{(k)}\right\}$ e $C_{\omega}=\left\{\omega^{(k)}\right\}$, que satisfaz

(1) $\omega^{(k)} \subset \Omega^{(k)} \subset \bar{\Omega} \quad$ e $\bigcup_{k} \omega^{(k)}=\bigcup_{k} \Omega^{(k)}=\bar{\Omega}$;

(2) Para qualquer $x$, existe $\omega^{(k)}$ tal que $x \in \omega^{(k)}$ e $\operatorname{dist}\left(x, \bar{\Omega} \backslash \omega^{(k)}\right) \geq \delta_{1}>0$;

(3) Para qualquer $\lambda$, existe um número $N_{0}$ que independe de $\lambda$ tal que $\bigcap_{k=1}^{N_{0}+1} \Omega^{(k)}=\emptyset ;$

(4) (a) se $\Omega^{(k)} \cap \partial \Omega=\emptyset$ (denotamos o conjunto de índices $k$ por $\left.\mathcal{M}\right)$, os $\omega^{(k)} \mathrm{e}$ $\Omega^{(k)}$ são cubos com o mesmo centro e com comprimento de seus lados $\frac{\lambda}{2}$ e $\lambda$, respectivamente;

(b) Se $\omega^{(k)} \cap \partial \Omega \neq \emptyset($ denotamos este conjunto de índices por $\mathcal{N})$, então para o sistema de coordenadas locais $\{y\}$ com centro $\xi^{(k)} \in S$,

$$
\begin{aligned}
\omega^{(k)} & =\left\{\left|y_{i}\right| \leq \frac{1}{2} \delta_{2} \lambda(i=1,2), 0 \leq y_{3}-F\left(y^{\prime} ; \xi^{k}\right) \leq \delta_{2} \lambda\right\} \\
\Omega^{(k)} & =\left\{\left|y_{i}\right| \leq \delta_{2} \lambda(i=1,2), 0 \leq y_{3}-F\left(y^{\prime} ; \xi^{k}\right) \leq 2 \delta_{2} \lambda\right\}
\end{aligned}
$$

onde $F\left(y^{\prime} ; \xi^{(k)}\right)\left(y^{\prime}=\left(y_{1}, y_{2}\right)\right)$ é a função que descreve a fronteira $S$ na vizinhança de $\xi^{(k)}$ e $\delta_{2}$ constante positiva que independe de $\lambda$.

Por mudança de variáveis de tal forma que $z_{i}=y_{i}(i=1,2)$ e $z_{3}=y_{3}-F\left(y^{\prime}\right)$, $\Omega^{(k)}(k \in \mathcal{N}), \Omega^{(k)}(k \in \mathcal{N})$ e a fronteira em $\Omega^{(k)}$ são respectivamente transformadas 
nos cubos padrões

$$
\begin{aligned}
\mathbb{K} & =\left\{\left|z_{i}\right| \leq \delta_{2} \lambda,(i=1,2), 0 \leq z_{3} \leq 2 \delta_{2} \lambda\right\} \\
\mathbb{K}^{\prime} & =\left\{\left|z_{i}\right| \leq \delta_{2} \lambda,(i=1,2), z_{3}=0\right\} .
\end{aligned}
$$

Além disso, sejam as partições da unidade $\left\{\zeta^{(k)}(x)\right\}$ e $\left\{\eta^{(k)}(x)\right\}$ subordinadas a $\left\{\Omega^{(k)}\right\}$ e $\left\{\omega^{(k)}\right\}$ respectivamente tal que

$$
\begin{aligned}
& \zeta^{(k)}(x)= \begin{cases}1, & \text { se } x \in \omega^{(k)}, \\
, & \text { se } x \in \bar{\Omega} \backslash \omega^{(k)}\end{cases} \\
& 0 \leq \zeta^{(k)}(x) \leq 1, \\
& \eta^{(k)}(x)=0 \quad \text { se } x \in \bar{\Omega} \backslash \Omega^{(k)}, \\
& \sum \zeta^{(k)}(x) \eta^{(k)}(x)=1, \\
& \left|D_{x}^{\alpha} \zeta^{(k)}(x)\right| \leq C_{\alpha} \lambda^{-|\alpha|},\left|D_{x}^{\alpha} \eta^{k}(x)\right| \leq C_{\alpha} \lambda^{-|\alpha|} .
\end{aligned}
$$

A função $\eta^{(k)}(x)$ pode ser construída a partir da função $\zeta^{(k)}(x)$, da seguinte maneira: pelas propriedades (2) e (3) das coberturas, temos que

$$
1 \leq \sum_{k}\left(\zeta^{(k)}(x)\right)^{2} \leq N_{0}
$$

Já que não tem mais de $N_{0}$ somandos diferentes de zero em cada ponto $x$; definimos então

$$
\eta^{(k)}=\frac{\zeta^{(k)}(x)}{\sum_{k}\left(\zeta^{(k)}(x)\right)^{2}} .
$$

Definamos agora o operador $L$ (com condições iniciais nulas) como sendo

$$
L(x, t, \partial x, \partial t) v=\rho(x, t) \frac{\partial v}{\partial t}-\nu \Delta v-\gamma \nabla \operatorname{div} v+4 \mu_{r} v
$$

e então definimos o operador

$$
A: W_{p}^{2,1}\left(Q_{\tau}\right) \mapsto L_{p}\left(Q_{\tau}\right) \times W_{p}^{2-1 / p}\left(\Sigma_{\tau}\right)
$$

por

$$
A v=\left[L(x, t, \partial x, \partial t) v,\left.v\right|_{\sum_{r}}\right]
$$


Portanto, a equação (2.4) pode ser escrita como

$$
A v=[g, 0]=h .
$$

Na notação usada acima, $V=W_{p}^{2,1}\left(Q_{\tau}\right)$ e $H=L_{p}\left(Q_{\tau}\right) \times W_{p}^{2-1 / p}\left(\Sigma_{\tau}\right)$ com norma

$$
\|h\|_{H}=\|(g, \phi)\|_{H}=\|g\|_{L_{p}\left(Q_{\tau}\right)}+\|\phi\|_{W_{p}^{2-1 / p}\left(\Sigma_{\tau}\right)} .
$$

Observe-se que $A$ assim definido é um operador limitado.

Agora passamos a construir o operador regularizador $R$. Para isto, seja

$$
L_{0}(x, t, \partial x, \partial t) v=\rho(x, t) \frac{\partial v}{\partial t}-\nu \Delta v-\gamma \nabla \operatorname{div} v
$$

e definamos $\bar{v}^{(k)}(x, t)$ (para $k \in \mathcal{M}$ ) como a solução do problema de valor inicial

$$
\begin{aligned}
& \rho\left(\xi^{k}, 0\right){\frac{\partial \bar{v}^{(k)}}{\partial t}}^{(k)}-\nu \bar{v}^{(k)}-\gamma \nabla \operatorname{div} \bar{v}^{k}=\hat{g}^{(k)}(x, t), \\
& \bar{v}^{(k)}(x, 0)=0,
\end{aligned}
$$

onde $\xi^{(k)}$ é o centro comum dos cubos $\omega^{(k)}$ e $\Omega^{(k)}$ e $\hat{g}^{(k)}(x, t)$ é definida por

$$
\hat{g}^{(k)}(x, t)= \begin{cases}\zeta^{(k)}(x) g(x, t), & x \in \Omega^{(k)}, \\ 0, & x \in \mathbb{R}^{3} \backslash \Omega^{(k)} .\end{cases}
$$

Para $k \in \mathcal{N}$, definamos $\bar{v}(z, t)$ como a solução do problema

$$
\begin{aligned}
& \rho\left(\xi^{(k)}, 0\right) \partial_{t} \bar{v}^{(k)}(z, t)-\nu \Delta \bar{v}^{(k)}-\gamma \nabla \operatorname{div} \bar{v}^{(k)}=\prod_{x}^{z} \zeta^{(k)}(x) g \\
& \left.\bar{v}^{(k)}(z, t)\right|_{z_{3}=0}=0 \\
& \bar{v}^{(k)}(z, 0)=0
\end{aligned}
$$

onde $\prod_{x}^{z}$ denota a transformação de $x$ a $z$.

Usando os resultados de [82], Cap. V, sabemos que o problema de Cauchy (2.10) possui uma única solução e satisfaz a estimativa

$$
\left\|\bar{v}^{(k)}\right\|_{W_{P}^{2,1}\left(\mathbb{R}^{3} \times[0, \tau]\right)} \leq C\left\|\bar{g}^{(k)}\right\|_{L_{p}\left(\mathbb{R}^{3} \times[0, \tau]\right)}
$$


onde as funções foram estendidas por zero fora de $\Omega^{(k)}$.

Similarmente, o problema (2.11) tem solução única e satisfaz

$$
\left\|\bar{v}^{(k)}(z, t)\right\|_{W_{p}^{2,1}\left(\mathbb{R}_{+}^{3} ;[0, \tau]\right)} \leq C\left\|\bar{g}^{(k)}\right\|_{L_{p}\left(\mathbb{R}_{+}^{3} \times[0, \tau]\right)} .
$$

Isto permite definir o regularizador $R$ como

$$
R h=\bar{v}^{(k)}(x, t)=\sum_{k \in \mathcal{M} \cup \mathcal{N}} \eta^{(k)}(x) v^{(k)}(x, t),
$$

onde $h=[g, \phi] \in L_{p}\left(Q_{\tau}\right) \times W_{p}^{2-1 / p}\left(\Sigma_{\tau}\right)$.

A demonstração de que $R$ é um operador limitado é análoga à demonstração de [82], onde é usado o fato de que as soluções dos problemas (2.10) e (2.11) satisfazem as estimativas acima, (2.12) e (2.13).

Agora vamos juntar num lema só alguns resultados de [82], que serão úteis nos cálculos a seguir.

Lema 2.6 Sejam $\tau=\lambda^{2} \chi, 0<\chi<1, \lambda^{2} \chi \leq T$,

$$
Q^{(k)}=\Omega^{(k)} \times[0, \tau]
$$

(i) No espaço $L_{p}\left(Q_{\tau}\right)$ a nova norma

$$
\|u\|_{L_{p}\left(Q_{\tau}\right)} \equiv\left(\sum_{k} \int_{Q^{(k)}}|u(x, t)|^{p} \mathrm{~d} x \mathrm{~d} t\right)^{1 / p}
$$

é equivalente à norma usual $\|u\|_{L_{p}\left(Q_{\tau}\right)}$ e a norma de $W_{p}^{2,1}\left(Q_{\tau}\right)$ definida por

$$
\|u\|_{W_{p}^{2,1}\left(Q_{\tau}\right)}=\left(\sum_{k}\|u\|_{W_{p}^{2,1}\left(Q^{(k)}\right)}\right)^{1 / p}
$$

é equivalente a $\|u\|_{W_{P}^{2,1}\left(Q_{\tau}\right)}$.

(ii) Se $u \in W_{p}^{2,1}\left(Q_{\tau}\right)$ tal que $u(x, 0)=0$ e $2 \mu+\nu<2$, então

$$
\left(\int_{Q^{(k)}}\left|D_{t}^{\mu} D_{x}^{\nu} u\right|^{p} \mathrm{~d} x \mathrm{~d} t\right)^{1 / p} \leq C \tau^{1-\frac{2 \mu+\nu}{2}} \sum_{2 \mu+\nu=2}\left(\int_{Q^{(k)}}\left|D_{t}^{\mu} D_{x}^{\nu} u\right|^{p} \mathrm{~d} x \mathrm{~d} t\right)^{1 / p}
$$


(iii) Se $u \in W_{p}^{2,1}\left(Q_{\tau}\right)$ tal que $u(x, 0)=0$ e se $\xi^{(k)}(x)$ é uma função definida sobre $\Omega^{(k)}$ satisfazendo a propriedade

$$
\left|D_{x}^{\mu} \xi^{(k)}(x)\right| \leq c \lambda^{-\nu}
$$

então para $\chi$ pequeno

$$
\sum_{2 \mu+\nu=2}\left(\int_{Q^{(k)}}\left|D_{t}^{\mu} D_{x}^{\nu} \zeta^{(k)}(x) u\right|^{p} \mathrm{~d} x \mathrm{~d} t\right)^{1 / p} \leq c \sum_{2 \mu+\nu=2}\left(\int_{Q^{(k)}}\left|D_{t}^{\mu} D_{x}^{\nu} u\right|^{p} \mathrm{~d} x \mathrm{~d} t\right)^{1 / p} .
$$

Ponhamos agora.

$$
L R h=L_{0} R h+L_{1} R h
$$

onde $L_{0}$ é o operador diferencial $L_{0}(x, t, \partial x, \partial t)$ definido acima e $L_{1}$ é o termo de baixa ordem. Avaliando $R h$ em $L_{0}$ obtemos

$$
\begin{aligned}
L_{0} R h= & \sum_{k} \eta^{(k)} L_{0}\left(\xi^{k}, 0, \partial x, \partial t\right) \bar{v}^{(k)} \\
& +\sum_{k} \eta^{(k)}\left[L_{0}(x, t, \partial x, \partial t)-L_{0}\left(\xi^{k}, 0, \partial x, \partial t\right)\right] \bar{v}^{(k)} \\
& +\sum_{k}\left[L_{0}\left(\eta^{(k)} v^{(k)}-\eta^{(k)} L_{0} v^{(k)}\right] .\right.
\end{aligned}
$$

Observemos que no primeiro somatório, para $k \in M$, obtém-se

$$
L_{0}\left(\xi^{(k)}, 0, \partial x, \partial t\right) \bar{v}^{(k)}(x, t)=\zeta^{(k)}(x) g(x, t)
$$

e observamos que para $k \in \mathcal{N}, x \in \Omega^{(k)}, \xi^{(k)} \in \partial \Omega$ e introduzindo as coordenadas locais $\{y\}$ obtém-se

$$
\begin{aligned}
& L_{0}\left(\xi^{(k)}, 0, \partial x, \partial t\right) \bar{v}^{(k)}(x, t)=\left.L_{0}\left(\xi^{(k)}, 0, \partial y, \partial t\right) \bar{v}^{(k)}(y, t)\right|_{y=y(x)} \\
& =\left.L_{0}\left(\xi^{(k)}, 0, \partial z, \partial t\right) \bar{v}^{(k)}(z, t)\right|_{z=\phi_{k}(y(x))}+\left[L_{0}\left(\xi^{(k)}, 0, \partial z-\nabla F \nabla_{3}, \partial t\right)\right. \\
& \left.\quad-\left.L_{0}\left(\xi^{(k)}, 0, \partial z, \partial t\right)\right|_{z=\phi_{k}(y(x))}\right]
\end{aligned}
$$

onde $z=\psi_{k}(y)$ está definida por $z_{1}=y_{1}, z_{2}=y_{2}, z_{3}=y_{3}-F\left(y_{1}, y_{2}\right)$ desde $\Omega^{(k)}$ ao cubo $\mathbb{K}=\left\{\left|z_{i}\right| \leq \delta_{0} \lambda, i=1,2,0 \leq z_{3} \leq 2 \delta_{0} \lambda\right\}$. Além disso, denotamos

$$
\nabla_{3}=\frac{\partial}{\partial z_{3}}, \quad \nabla F=\left(\frac{\partial F}{\partial z_{1}}, \frac{\partial F}{\partial z_{2}}, 0\right)
$$




$$
\begin{aligned}
\frac{\partial}{\partial y_{i}} & =\frac{\partial}{\partial z_{i}}-\frac{\partial F}{\partial z_{i}} \cdot \nabla_{3}, \quad i \neq 3, \\
\frac{\partial}{\partial y_{3}} & =\frac{\partial}{\partial z_{3}} .
\end{aligned}
$$

Portanto, substituindo (2.15), (2.16) em (2.14) e levando em conta que o primeiro termo do lado direito é igual a $\bar{g}(z, t)$, obtém-se

$$
\begin{aligned}
L_{0} R h= & \sum_{k \in \mathcal{M}} \eta^{(k)}(x) \zeta^{(k)}(x) g+\sum_{k \in \mathcal{N}} \eta^{(k)} \zeta^{(k)}(x) g \\
& +\sum_{k \in \mathcal{N}} \eta^{(k)}(x)\left[L_{0}\left(\xi^{(k)}, 0, \partial z-\nabla F \cdot \nabla_{3}, \partial t\right)-L_{0}\left(\xi^{(k)}, 0, \partial z, \partial t\right)\right] \bar{v}^{(k)}(z, t) \\
& +\sum_{k}\left[L_{0}\left(\eta^{(k)} \bar{v}\right)-\eta^{(k)} L_{0} \bar{v}^{(k)}\right] \\
= & g+\sum_{k \in \mathcal{N}} \eta^{(k)}(x)\left[L_{0}\left(\xi^{(k)}, 0, \partial z-\nabla F \cdot \nabla_{3}, \partial t\right)-L_{0}\left(\xi^{(k)}, 0, \partial z, \partial t\right)\right] \bar{v}^{(k)}(z, t) \\
& +\sum_{k}\left[L_{0}\left(\eta^{(k)} \bar{v}^{(k)}\right)-\eta^{(k)} L_{0}\left(\bar{v}^{(k)}\right)\right] .
\end{aligned}
$$

Assim, $L R h=g+T_{1} h$, onde o operador $T_{1} h$ é definido por

$$
\begin{aligned}
T_{1} h= & \left(L_{1} R h+\sum_{k}\left(L_{0}\left(\eta^{(k)} \bar{v}^{(k)}\right)-\eta^{k} L_{0}\left(\bar{v}^{(k)}\right)\right.\right. \\
& +\sum_{k} \eta^{(k)}\left[L_{0}(x, t, \partial x, \partial t)-L_{0}\left(\xi^{(k)}, 0, \partial x, \partial t\right)\right] \bar{v}^{(k)} \\
& \left.+\sum_{k \in \mathcal{N}} \eta^{(k)}\left[L_{0}\left(\xi^{(k)}, 0, \partial z-\nabla F \cdot \nabla_{3}, \partial / \partial t\right)-L_{0}\left(\xi^{(k)}, 0, \partial z, \partial t\right)\right] \bar{v}^{(k)}(z, t), 0\right) .
\end{aligned}
$$

Observe-se que

$$
\begin{aligned}
& L_{0}\left(\eta^{(k)} \bar{v}^{(k)}\right)-\eta^{(k)} L_{0}\left(\bar{v}^{(k)}\right)=\rho(x, t) \frac{\partial}{\partial t}\left(\eta^{(k)} \bar{v}^{(k)}\right)-\nu \Delta\left(\eta^{(k)} \bar{v}^{(k)}\right) \\
& -\rho(x, t) \eta^{(k)} \frac{\partial}{\partial t} \bar{v}^{(k)}+\nu \eta^{(k)} \Delta v^{(k)}+\gamma \eta^{(k)} \nabla \operatorname{div} \bar{v}^{(k)}-\gamma \nabla \operatorname{div}\left(\eta^{(k)} \bar{v}^{(k)}\right) \\
= & -(\nu+\gamma) \Delta \eta^{(k)} \bar{v}^{(k)}-(2 \nu+\gamma) \nabla \eta^{(k)} \nabla \bar{v}^{(k)}-\gamma \nabla \eta^{(k)} \operatorname{div} \bar{v}^{(k)} .
\end{aligned}
$$

Similarmente,

$$
\left[L_{0}(x, t, \partial x, \partial t)-L_{0}\left(\xi^{(k)}, 0, \partial x, \partial t\right)\right] \bar{v}^{(k)}=\left[\rho(x, t)-\rho\left(\xi^{(k)}, 0\right)\right] \partial t \bar{v}^{(k)}
$$

Finalmente, o último somatório do operador $T_{1}$,

$$
\left[L_{0}\left(\xi^{(k)}, 0, \partial z-\nabla F \nabla_{3}, \partial t\right)-L\left(\xi^{(k)}, 0, \partial z, \partial t\right)\right] \bar{v}^{(k)}(z, t)
$$




$$
\begin{aligned}
= & \rho\left(\xi^{(k)}, 0\right) \frac{\partial \bar{v}^{(k)}}{\partial t}-\mu\left(\partial z-\nabla F \nabla_{3}\right)^{2}-\gamma\left(\partial z-\nabla F \nabla_{3}\right) \operatorname{div}_{z}\left(\bar{v}^{(k)}(z, t)\right) \\
& -\rho\left(\xi^{(k)}, 0\right) \partial_{t} \bar{v}^{(k)}(z, t)+\mu \Delta \bar{v}^{(k)}(z, t)+\nabla \operatorname{div}\left(\bar{v}^{(k)}(z, t)\right) .
\end{aligned}
$$

Já que

$$
\begin{aligned}
& \left(\partial z-\nabla F \nabla_{3}\right)\left(\partial z-\nabla F \nabla_{3}\right) \bar{v}^{(k)}(z, t)=\Delta \bar{v}^{(k)}(z, t)+(\nabla F)^{2} \nabla_{3}^{2} \bar{v}^{(k)}(z, t) \\
& -\nabla^{2} F \nabla_{3} \bar{v}^{(k)}(z, t)-2 \nabla F \nabla \nabla_{3} \bar{v}^{(k)}
\end{aligned}
$$

e

$$
\begin{aligned}
& \left(\nabla-\nabla F \nabla_{3}\right)\left(\operatorname{div}\left(\bar{v}^{(k)}(z, t)-\frac{\partial F}{\partial z_{1}} \nabla_{3} v_{1}-\frac{\partial F}{\partial z_{2}} \nabla_{3} v_{2}\right)\right. \\
& =\nabla\left(\operatorname{div} \bar{v}^{(k)}\right)-\nabla^{2} F \nabla_{3} \bar{v}^{(k)}-\nabla F \nabla \nabla_{3} \bar{v}^{(k)}-\nabla F \nabla_{3}\left(\operatorname{div} \bar{v}^{(k)}\right)+(\nabla F)^{2} \nabla_{3}^{2} \bar{v}^{(k)},
\end{aligned}
$$

substituindo (2.20) e (2.21) em (2.19) obtém-se

$$
\begin{aligned}
& {\left[L_{0}\left(\xi^{(k)}, 0, \partial z-\nabla F \nabla_{3}, \partial t\right)-L\left(\xi^{(k)}, 0, \partial z, \partial t\right)\right] \bar{v}^{(k)}(z, t)} \\
& =(\nu+\gamma) \nabla^{2} F \nabla_{3} \bar{v}^{(k)}(z, t)+(2 \mu+\gamma) \nabla F \nabla \nabla_{3} \bar{v}^{(k)}(z, t) \\
& \quad-(\nu+\gamma)(\nabla F)^{2} \nabla_{3}^{2} \bar{v}^{(k)}(z, t) .
\end{aligned}
$$

Finalmente, usando a última expressão, (2.17), (2.18), obtém-se que

$$
\begin{aligned}
T_{1} h= & \left(L_{1} R h+\sum_{k \in \mathcal{N}} \eta^{(k)}(x) \Pi_{z}^{x}\left\{(\nu+\gamma) \nabla^{2} F \nabla_{3} \bar{v}^{(k)}(z, t)\right.\right. \\
& +(2 \nu+\gamma) \nabla F \cdot \nabla \nabla_{3} \bar{v}^{(k)}(z, t)-(\nu+\gamma)(\nabla F)^{2} \nabla_{3}^{2} \bar{v}^{(k)}(z, t) \\
& \left.+\gamma \nabla F \nabla_{3}\left(\operatorname{div} \bar{v}^{(k)}(z, t)\right)\right\}+\sum_{k} \eta^{(k)}\left[\rho(x, t)-\rho\left(\xi^{(k)}, 0\right)\right] v^{(k)}(z, t) \\
& \left.+\sum_{k}\left[-(\nu+\gamma) \Delta \eta^{(k)} \bar{v}^{(k)}+(2 \nu+\gamma) \nabla \eta^{(k)} \nabla \bar{v}^{(k)}-\gamma \nabla \eta^{(k)} \operatorname{div} \bar{v}^{(k)}\right], 0\right) .
\end{aligned}
$$

Agora vamos calcular a norma de $T_{1}$. Devido ao fato de que $\rho(x, t) \in C^{\alpha, \beta}\left(\bar{Q}_{T}\right)$, temos

$$
\begin{aligned}
\left|\rho(x, t)-\rho\left(\xi^{(k)}, 0\right)\right| & \leq\left|\rho(x, t)-\rho\left(\xi^{(k)}, t\right)\right|+\left|\rho\left(\xi^{(k)}, t\right)-\rho\left(\xi^{(k)}, 0\right)\right| \\
& \leq C_{0}\left|x-\xi^{(k)}\right|^{\alpha}+C_{1} \tau^{\beta} \\
& \leq C\left(\lambda^{\alpha}+\lambda^{2 \beta} \chi^{\beta}\right),
\end{aligned}
$$


pois $\left|x-\xi^{(k)}\right| \leq \lambda$.

Usando o Lema 2.6, as propriedades (2.9) das funções suaves e a propriedade (3) das coberturas, temos

$$
\begin{aligned}
& \sum_{k} \|\left[-(2 \nu+\gamma) \nabla \eta^{(k)} \nabla \bar{v}^{(k)}-(\nu+\gamma) \Delta \eta^{(k)} \bar{v}^{(k)}-\gamma \nabla \eta^{(k)} \operatorname{div} \bar{v}^{(k)} \|_{L_{p}\left(Q^{(k)}\right)}^{p}\right. \\
\leq & C(2 \nu+\gamma) \lambda^{-1} \sum_{k}\left\|\nabla \bar{v}^{(k)}\right\|_{L_{p}\left(Q^{(k)}\right)}^{p}+\left(C(\nu+\gamma) \lambda^{-2}\right)^{p} \sum_{k}\left\|\bar{v}^{(k)}\right\|_{L_{p}\left(Q^{(k)}\right)}^{p} \\
& +\left(C \tau^{1 / 2} \gamma \lambda^{-1}\right)^{p} \sum_{k}\left(\left\|D_{x}^{2} \bar{v}^{(k)}\right\|_{L_{p}\left(Q^{(k)}\right)}^{p}+\left\|\partial_{t} \bar{v}^{(k)}\right\|_{L_{p}\left(Q^{(k)}\right)}^{p}\right) \\
\leq & C\left(2 \lambda^{-1} \tau^{1 / 2}+\lambda^{-2} \tau\right)^{p}(\nu+\gamma)^{p}\left(\sum_{k}\left\|D_{x}^{2} \bar{v}^{(k)}\right\|_{L_{p}\left(Q^{(k)}\right)}^{p}+\left\|\partial_{t} \bar{v}^{(k)}\right\|_{L_{p}\left(Q^{(k)}\right)}^{p}\right) \\
\leq & C(\nu, \gamma)\left(2 \lambda^{-1} \tau^{1 / 2}+\lambda^{-2} \tau\right)\left\|g^{(k)}(x, t)\right\|_{L_{p}\left(Q_{t}\right)},
\end{aligned}
$$

devido à estimativa (2.12). Similarmente,

$$
\begin{aligned}
& \sum_{k \in N} \| \eta^{(k)}(x) \Pi_{z}^{x}\left\{(\nu+\gamma) \nabla^{2} F \nabla_{3} \bar{v}^{(k)}(\bar{z}, t)+(2 \nu+\gamma) \nabla F \cdot \nabla \nabla_{3} \bar{v}^{(k)}(z, t)\right. \\
& \left.-(\nu+\gamma)(\nabla F)^{2} \nabla_{3}^{2} \bar{v}^{(k)}(z, t)+\gamma \nabla F \nabla_{3}\left(\operatorname{div} \bar{v}^{(k)}\right)\right\} \|_{L_{p}\left(Q^{(k)}\right)} \\
\leq & \left(C(\nu+\gamma) \lambda^{-2}\right)^{p} \sum_{k}\left\|D_{x}^{2} \bar{v}^{(k)}\right\|_{L_{p}\left(Q^{(k)}\right)}+\left(C(2 \nu+\gamma) \lambda^{-1}\right)^{p} \sum_{k}\left\|D_{x}^{2} \bar{v}^{(k)}\right\|_{L_{p}\left(Q^{(k)}\right)}^{p} \\
& +\left(C(\nu+\gamma) \lambda^{-2}\right)^{p} \sum_{k}\left\|D_{x}^{2} \bar{v}^{(k)}\right\|_{L_{p}\left(Q^{(k)}\right)}^{p}+\gamma C \lambda^{-1} \sum_{k}\left\|D_{x}^{2} \bar{v}^{(k)}\right\|_{L_{p}\left(Q^{(k)}\right)}^{p} \\
\leq & C(\nu+\gamma) \lambda^{-2} \sum_{k}\left\|\nabla \bar{v}^{(k)}\right\|_{L_{p}\left(Q^{(k)}\right)}+C(\nu+\gamma)\left(2 \lambda^{-1}+\lambda^{-2}\right) \sum_{k}\left\|D_{x}^{2} \bar{v}^{(k)}\right\|_{L_{p}\left(Q^{(k)}\right)} \\
\leq & \left(C(\nu+\gamma)\left(2 \lambda^{-1}+\lambda^{-2}\right)\right)^{p} \sum_{k}\left\|\tilde{g}^{(k)}(z, t)\right\|_{L_{p}\left(Q^{(k)}\right)}^{p} \| \sum_{k \in}[\rho(x, t) \\
& \left.-\rho\left(\xi^{(k)}, 0\right)\right] \partial_{t} \bar{v}^{(k)} \|_{L_{p}\left(Q^{(k)}\right)}^{p} \\
\leq & C\left(\lambda^{\alpha}+\lambda^{2 \beta} \chi\right)^{p}\left\|\partial t \bar{v}^{(k)}\right\|_{L_{p}\left(Q^{(k)}\right)}^{p}\left\|L_{1} R h\right\|_{L_{p}\left(Q^{(k)}\right)} \\
\leq & C\left\|L_{1} v^{(k)}\right\|_{L_{p}\left(Q^{(k)}\right)} \leq C \tau\left\|\bar{v}^{(k)}\right\|_{W_{p}^{2,1}} \leq C \tau\left\|g^{(k)}\right\|_{L_{p}\left(Q^{(k)}\right)} .
\end{aligned}
$$

Portanto,

$$
\begin{aligned}
\left\|T_{1} h\right\|_{L_{p}(Q \tau)} \leq & C\left\{\left(\lambda^{\alpha}+\lambda^{2 \beta} \chi\right)+\tau+(\nu+\gamma)\left(2 \lambda^{-1}+\lambda^{-2}\right)\right. \\
& \left.+\left(2 \lambda^{-1} \tau^{1 / 2}+\lambda^{-2} \tau\right)\right\}\|g\|_{L_{P}\left(Q_{\tau}\right)} .
\end{aligned}
$$


Agora podemos escolher $\lambda, \tau, \chi$ de tal forma que $\left\|T_{1}\right\|<1$. Portanto, a solução $v$ sobre o intervalo $[0, \tau]$ pode ser encontrada na forma

$$
v=R\left(I+T_{1}+T_{1}^{2}+\cdots\right)=R\left(I-T_{1}\right)^{-1} h .
$$

Nota: Similarmente, podemos demonstrar que o operador $M$ possui norma menor que 1.

Como nossa prova foi feita para $[0, \tau] \operatorname{com} \tau$ pequeno, precisamos estender o argumento para $[0, T]$. Queremos resolver para $[\tau, 2 \tau]$.

Definamos a função

$$
v^{*}(x, t)= \begin{cases}v(x, t) & \text { para } 0 \leq t \leq \tau \\ v(x, 2 \tau-t) & \text { para } \tau \leq t \leq 2 \tau .\end{cases}
$$

Seja $\tilde{v}(x, t)$ a solução do problema abaixo, com

$$
\begin{gathered}
F(x, t)=g(x, t)-g(x, 2 \tau)+\rho(x, t) v_{t}(x, 2 \tau-t)+\rho(x, 2 \tau-t) v_{t}(x, 2 \tau-t), \\
\rho \tilde{v}_{t}-\nu \Delta \tilde{v}-\gamma \nabla \operatorname{div} \tilde{v}=F(x, t), \\
\left.\tilde{v}\right|_{\tilde{\Sigma}_{2 \tau}}=0 \\
\left.\tilde{v}\right|_{t=0}=0
\end{gathered}
$$

para $\tau \leq t \leq 2 \tau, \tilde{\Sigma}_{2 \Gamma}=\partial \Omega \times[\tau, 2 \tau]$ e $\tilde{v} \equiv 0$ para $0 \leq t \leq \tau$. Então podemos verificar que $v=v^{k}+\tilde{v}$ é solução do problema abaixo.

$$
\begin{aligned}
& \rho v_{t}-\nu \Delta v-\gamma \nabla \operatorname{div} v=g, \\
& \left.v\right|_{\Sigma_{2 \tau}}=0, \\
& \left.v\right|_{t=0}=0 .
\end{aligned}
$$

A estimativa (2.3) foi obtida por Solonnikov [83] para domínios limitados. No entanto, se usarmos a propriedade 3 do sistema de cobertura, esta permanece válida para domínios não limitados. 


\subsection{Aproximações auxiliares}

Vamos construir a solução aproximada indutivamente como sendo

$$
u^{(0)}=0, \quad w^{(0)}=0
$$

e definimos para $k=1,2,3, \ldots,\left\{\rho^{(k)}\right\},\left\{u^{(k)}, p^{(k)}\right\}$ e $\left\{\omega^{(k)}\right\}$ como as respectivas soluções dos problemas

$$
\begin{aligned}
& \left\{\begin{array}{l}
\frac{\partial \rho^{(k)}}{\partial t}+\left(u^{(k-1)} \cdot \nabla\right) \rho^{(k)}=0, \\
\rho^{(k)}(x, 0)=\rho_{0}(x),
\end{array}\right.
\end{aligned}
$$

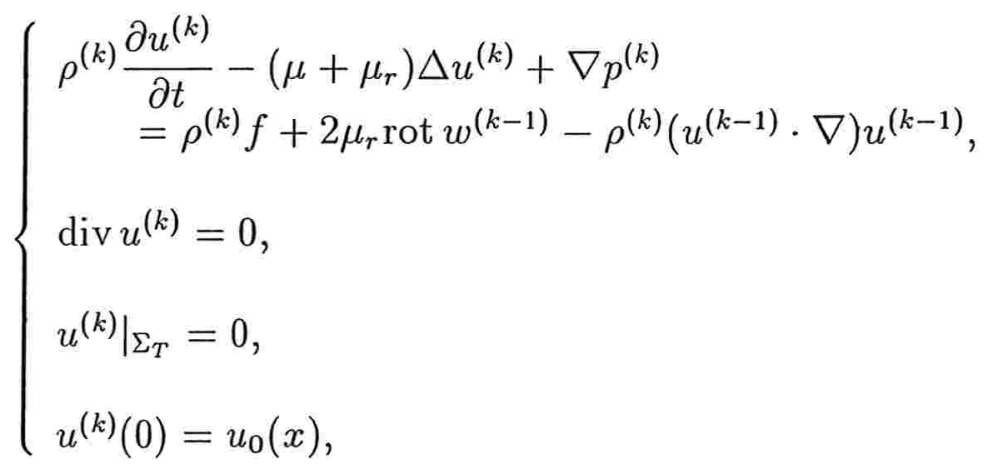

$$
\begin{aligned}
& \left\{\begin{array}{l}
\rho^{(k)} \frac{\partial w^{(k)}}{\partial t}-\nu \Delta w^{(k)}-\gamma \nabla \operatorname{div} w^{(k)}+4 \mu_{r} w^{(k)} \\
\quad=\rho^{(k)} g+2 \mu_{r} \operatorname{rot} u^{(k-1)}-\rho^{(k)}\left(u^{(k-1)} \cdot \nabla\right) w^{(k-1)} \\
\left.w^{(k)}\right|_{\Sigma_{T}}=0, \\
w^{(k)}(0)=w_{0}(x) .
\end{array}\right.
\end{aligned}
$$

O próximo lema tem por objetivo mostrar que as seqüências de soluções dos problemas acima são limitadas.

Lema 2.7 Para $T_{1} \in(0, T]$ suficientemente pequeno, a seqüência $\left\{u^{(k)}, \nabla p^{(k)}, w^{(k)}\right\}$ é limitada no espaço de Banach $W_{p}^{2,1}\left(Q_{T_{1}}\right) \times L_{p}\left(Q_{T_{1}}\right) \times W_{p}^{2,1}\left(Q_{T_{1}}\right)$. 
Demonstração: Ponhamos

$$
\phi^{(k)}(T)=\left\|u^{(k)}\right\|_{W_{p}^{2,1}\left(Q_{T}\right)}+\left\|w^{(k)}\right\|_{W_{p}^{2,1}\left(Q_{T_{1}}\right)}+\left\|\nabla p^{(k)}\right\|_{L_{p}\left(Q_{T}\right)}
$$

Como conseqüência da aplicação dos lemas da seção anterior aos problemas (2.23) $-(2.25)$, temos

$$
\begin{aligned}
& \phi^{(k)}(T) \leq K_{1}\left(\|\rho\|_{C^{\alpha, \beta}, T}\right)\left(\left\|u_{0}\right\|_{W_{p}^{2-2 / p}(\Omega)}+\left\|\rho^{(k)} f\right\|_{L_{p}\left(Q_{T}\right)}\right. \\
& +\left\|\rho^{(k)}\left(u^{(k-1)} \cdot \nabla\right) u^{(k-1)}\right\|_{L_{p}\left(Q_{T}\right)}+2 \mu_{r}\left\|\operatorname{rot} w^{(k)}\right\|_{L_{p}\left(Q_{T}\right)} \\
& +K_{2}\left(\|\rho\|_{C^{\alpha, \beta}}, T\right)\left(\left\|w_{0}\right\|_{W_{p}^{2-2 / p}(\Omega)}+\left\|\rho^{(k)} g\right\|_{L_{p}\left(Q_{T}\right)}\right. \\
& \left.+\left\|\rho^{(k)}\left(u^{(k-1)} \cdot \nabla\right) w^{(k-1)}\right\|_{L_{p}\left(Q_{T}\right)}+2 \mu_{r}\left\|\operatorname{rot} u^{(k-1)}\right\|_{L_{p}\left(Q_{T}\right)}\right) .
\end{aligned}
$$

Para estimar o termo $\left\|\rho^{(k)}\left(u^{(k-1)} \cdot \nabla\right) u^{(k-1)}\right\|_{L_{p}\left(Q_{T}\right)}$ primeiro vamos obter a desigualdade

$$
\left\|u^{(k-1)}\right\|_{L^{\infty}\left(Q_{T}\right)} \leq C_{1}\left(\left\|u_{0}\right\|_{W_{P}^{2-2 / p}}+\left\|w_{0}\right\|_{W_{p}^{2-2 / p}(\Omega)}+T^{(1-1 / p)(1-3 / p)} \phi^{(k-1)}(T)\right) .
$$

Como

$$
\left\|u^{(k-1)}(t)\right\|_{L_{\infty}(\Omega)} \leq\left\|u^{(k-1)}(t)-u_{0}\right\|_{L_{\infty}(\Omega)}+\left\|v_{0}\right\|_{L_{\infty}(\Omega)}
$$

usando a desigualdade do Teorema $1.3 \operatorname{com} p=\infty, q=r, a=3 / p$ e o fato de que $\left.u^{(k-1)}(t)\right|_{\partial \Omega}=0,\left.u_{0}(x)\right|_{\partial \Omega}=0$, obtém-se

$$
\left\|u^{(k-1)}(t)-u_{0}\right\|_{L^{\infty}(\Omega)} \leq C_{2}\left\|u^{(k-1)}(t)-u_{0}\right\|_{W_{p}^{1}(\Omega)}^{3 / p}\left\|u^{(k-1)}(t)-u_{0}\right\|_{L_{p}(\Omega)}^{1-3 / p} .
$$

Além disto,

$$
\begin{aligned}
\left\|u^{(k-1)}(t)-u_{0}\right\|_{L_{p}(\Omega)}^{p} & =\int_{\Omega}\left|u^{(k-1)}(t)-u_{0}\right|^{p} \mathrm{~d} x \\
& =\int_{\Omega}\left|\int_{0}^{t} u_{t}^{(k-1)}(s) \mathrm{d} s\right|^{p} \mathrm{~d} x \\
& \leq \int_{\Omega}\left|\left(\int_{0}^{t} \mathrm{~d} s\right)^{1 / q}\left(\int_{0}^{t}\left|u_{t}^{(k-1)}(s)\right|^{p} \mathrm{~d} s\right)^{1 / p}\right|^{p} \mathrm{~d} x \\
& \leq t^{p / q}\left\|u^{(k-1)}\right\|_{W_{P}^{2,1}\left(Q_{T}\right)}^{p}
\end{aligned}
$$


onde $\frac{1}{p}+\frac{1}{q}=1$.

Fazendo uso da seguinte imersão tipo Sobolev, ver Adams [1, p. 108], para $m p>N$, temos

$$
W_{p}^{m}(\Omega) \hookrightarrow L_{q}(\Omega), \quad p \leq q \leq \infty
$$

Obtemos

$$
\left\|u_{0}\right\|_{L_{\infty}(\Omega)} \leq C_{2}\left\|u_{0}\right\|_{W_{p}^{1}(\Omega)} \leq C_{3}\left\|u_{0}\right\|_{W_{p}^{2-2 / p}(\Omega)}
$$

Usando a desigualdade de Solonnikov (1.2) obtém-se

$$
\begin{aligned}
\sup _{0 \leq t \leq T}\left\|u^{(k-1)}\right\|_{W_{p}^{1}(\Omega)} & \leq \sup _{0 \leq t \leq T}\left\|u^{(k-1)}(t)\right\|_{W_{P}^{2-2 / P}(\Omega)} \\
& \leq C_{4}\left\|u^{(k-1)}\right\|_{W_{P}^{2,1}\left(Q_{T}\right)}+\left\|u_{0}\right\|_{W_{P}^{2-2 / p}(\Omega)}
\end{aligned}
$$

Substituindo as desigualdades (2.29), (2.31), (2.32) em (2.28), obtém-se o resultado, ou seja, a desigualdade (2.27).

Agora vamos limitar (2.26). Usando (2.30), vem que

$$
\begin{aligned}
\int_{0}^{T}\left\|\nabla u^{(k-1)}(t)\right\|_{L_{\infty}(\Omega)} & \leq C_{5} \int_{0}^{T}\left\|u^{(k-1)}(t)\right\|_{W_{p}^{2}(\Omega)} \mathrm{d} t \\
& \leq C_{5}\left(\int_{0}^{T} \mathrm{~d} t\right)^{1 / q}\left(\int_{0}^{T}\left\|u^{(k-1)}(t)\right\|_{W_{p}^{2}(\Omega)}^{p} \mathrm{~d} t\right)^{1 / p} \\
& \leq C_{5} T^{1 / q} \Phi^{(k-1)}(T) .
\end{aligned}
$$

Usando a desigualdade do Teorema 1.3 do Capítulo 1,

$$
\begin{aligned}
\left\|\nabla u^{(k-1)}(t)\right\|_{L_{P}(\Omega)} & \leq\left\|u^{(k-1)}\right\|_{W_{P}^{1}(\Omega)} \\
& \leq\left\|u^{(k-1)}(t)\right\|_{W_{p}^{2}(\Omega)}^{a}\left\|u^{(k-1)}(t)\right\|_{L^{\infty}(\Omega)}^{(1-a)} .
\end{aligned}
$$

Temos que

$$
\begin{gathered}
\left\|\rho^{(k)}\left(u^{(k-1)} \cdot \nabla\right) u^{(k-1)}\right\|_{L_{P}\left(Q_{T}\right)}^{p} \leq M^{p}\left\|u^{(k-1)}\right\|_{L_{\infty}\left(Q_{T}\right)}^{p} \int_{0}^{T}\left\|\nabla u^{(k-1)}\right\|_{L_{P}(\Omega)}^{p} \mathrm{~d} t \\
\leq M^{p}\left\|u^{(k-1)}\right\|_{L^{\infty}\left(Q_{T}\right)}^{p} \int_{0}^{T}\left\|u^{(k-1)}\right\|_{W_{P}^{2}(\Omega)}^{a p}\left\|u^{(k-1)}(t)\right\|_{L^{\infty}(\Omega)}^{(1-a) p} \mathrm{~d} t
\end{gathered}
$$




$$
\begin{aligned}
& \leq M^{p}\left\|u^{(k-1)}\right\|_{L^{\infty}\left(Q_{T}\right)}^{p} \int_{0}^{T}\left\|u^{(k-1)}\right\|_{W_{p}^{2}(\Omega)}^{a p}\left\|u^{(k-1)}(t)\right\|_{L^{\infty}(\Omega)}^{(1-a) p} \mathrm{~d} t \\
& \leq C M^{p}\left\|u^{(k-1)}\right\|_{L_{\infty}\left(Q_{T}\right)}^{(2-a) p} \int_{0}^{T}\left\|u^{(k-1)}(t)\right\|_{W_{p}^{2}(\Omega)}^{a p} \mathrm{~d} t \\
& \leq C M^{p}\left\|u^{(k-1)}\right\|_{L_{\infty}\left(Q_{T}\right)}^{(2-a) p} T^{(1-a)}\left(\int_{0}^{T}\left\|u^{(k-1)}\right\|_{W_{p}^{2}(\Omega)}^{p} \mathrm{~d} t\right)^{a} \\
& \leq C M^{p}\left\|u^{(k-1)}\right\|_{L^{\infty}\left(Q_{T}\right)}^{(2-a) p} T^{(1-a)}\left\|u^{(k-1)}\right\|_{W_{P}^{2,1}\left(Q_{T}\right)^{a}}^{a p}
\end{aligned}
$$

Portanto, obtém-se

$$
\left\|\rho^{(k)}\left(u^{(k-1)} \cdot \nabla\right) u^{(k-1)}\right\|_{L_{p}\left(Q_{T}\right)} \leq C M\left\|u^{(k-1)}\right\|_{L^{\infty}\left(Q_{T}\right)}^{(2-a)} \cdot T^{\frac{1-a}{p}}\left(\phi^{(k-1)}(T)\right)^{a} .
$$

Usando a desigualdade (2.27) junto com a de Young, com $\alpha=a / 2$, obtém-se

$$
\begin{aligned}
& \left\|\rho^{(k)}\left(u^{(k-1)} \cdot \nabla\right) u^{(k-1)}\right\|_{L_{p}\left(Q_{T}\right)} \leq C M T^{\frac{(1-a)}{p}}\left(\phi^{(k-1)^{2}}(T)\right)^{\frac{a}{2}}\left(\left\|u_{0}\right\|_{W_{p}^{2-2 / p}(\Omega)}+\right. \\
& \left.\left\|w_{0}\right\|_{W_{p}^{2-2 / p}(\Omega)}+T^{(1-1 / p)(1-3 / p)} \phi^{(k-1)}(T)\right)^{2\left(\frac{2-a}{2}\right)} \\
\leq & \alpha \varepsilon \phi^{(k-1)}(T)^{2}+(1-\alpha) \varepsilon^{-\frac{\alpha}{1-\alpha}}\left(\left\|u_{0}\right\|_{W_{p}^{2-2 / p}(\Omega)}+\left\|w_{0}\right\|_{W_{p}^{2-2 / p}(\Omega)}\right. \\
& \left.+T^{(1-1 / p)(1-3 / p)} \phi^{(k-1)}(T)\right)^{2}\left(C M T^{\frac{1-a}{p}}\right)^{\frac{1}{1-\alpha}} .
\end{aligned}
$$

Escolhendo $\varepsilon=\left(T^{\frac{1-a}{p}}\right)^{\frac{1}{\alpha}}$ e depois de manipular os termos, obtemos finalmente $\left\|\rho^{(k)}\left(u^{(k-1)} \cdot \nabla\right) u^{(k-1)}\right\|_{L_{p}\left(Q_{T}\right)} \leq C M\left(\left(\left\|u_{0}\right\|_{W_{P}^{2-2 / p}(\Omega)}^{2}+\left\|w_{0}\right\|_{W_{P}^{2-2 / p}(\Omega)}^{2}\right)+T^{\delta} \phi^{(k-1)}(T)^{2}\right)$.

Agora vamos limitar os rotacionais.

$$
\begin{aligned}
\left\|\operatorname{rot} w^{(k-1)}\right\|_{L_{p}(\Omega)} & \leq C\left\|\nabla w^{(k-1)}\right\|_{L_{p}(\Omega)} \\
& \leq C\left\|w^{(k-1)}\right\|_{W_{P}^{2}(\Omega)}^{a}\left\|w^{(k-1)}\right\|_{L_{p}(\Omega)}^{(1-a)} .
\end{aligned}
$$

Agora

$$
\begin{aligned}
\int_{0}^{T}\left\|w^{(k-1)}(t)\right\|_{W_{p}^{2}(\Omega)}^{a p} \mathrm{~d} t & \leq T^{(1-a)}\left(\int_{0}^{T}\left\|w^{(k-1)}(t)\right\|_{W_{p}^{2}(\Omega)}^{p} \mathrm{~d} t\right)^{a} \\
& \leq T^{(1-a)}\left\|w^{(k-1)}\right\|_{W_{p}^{2,1}\left(Q_{T}\right)}^{a p} \\
& \leq T^{(1-a)}\left(\Phi^{(k-1)}(T)\right)^{a p}
\end{aligned}
$$


Portanto, obtém-se

$$
\begin{aligned}
\left\|\operatorname{rot} w^{(k-1)}\right\|_{L_{p}\left(Q_{T}\right)}^{p} & =\int_{0}^{T} \int_{\Omega}\left|\operatorname{rot} w^{(k-1)}\right|^{p} \mathrm{~d} x \mathrm{~d} t \\
& =\int_{0}^{T}\left\|\operatorname{rot} w^{(k-1)}\right\|_{L_{p}(\Omega)}^{p} \mathrm{~d} t \\
& \leq C \int_{0}^{T}\left\|\nabla w^{(k-1)}\right\|_{L_{p}(\Omega)}^{p} \mathrm{~d} t \\
& \leq C \int_{0}^{T}\left\|w^{(k-1)}(t)\right\|_{L^{\infty}(\Omega)}^{(1-a)}\left\|w^{(k-1)}(t)\right\|_{W_{p}^{2}(\Omega)}^{a p} \mathrm{~d} t \\
& \leq C\left\|w^{(k-1)}(t)\right\|_{L_{p}\left(Q_{T}\right)}^{(1-a) p} T^{\frac{(1-a)}{p}}\left(\phi^{(k-1)}(T)\right)^{a p} .
\end{aligned}
$$

Logo, aplicando a desigualdade de Young outra vez,

$$
\begin{aligned}
& \left\|\operatorname{rot} w^{(k-1)}\right\|_{L_{p}\left(Q_{T}\right)} \leq C\left\|w^{(k-1)}(t)\right\|_{L^{\infty}\left(Q_{T}\right)}^{(1-a)} T^{\frac{(1-a)}{p}}\left(\phi^{(k-1)}(T)\right)^{a} \\
\leq & C T^{\frac{1-a}{p}}\left(\phi^{(k-1)}(T)\right)^{a}\left(\left\|u_{0}\right\|_{W_{p}^{2-2 / p}(\Omega)}+\left\|w_{0}\right\|_{W_{p}^{2-2 / p}(\Omega)}+T^{(1-1 / p)(1-3 / p)} \phi^{(k-1)}(T)\right)^{(1-a)} \\
= & a \varepsilon \phi^{(k-1)}(T)+(1-a) \varepsilon^{-\frac{a}{1-a}}\left(\left\|u_{0}\right\|_{W_{p}^{2-2 / p}(\Omega)}+\left\|w_{0}\right\|_{W_{p}^{2-2 / p}(\Omega)}\right. \\
& \left.+T^{(1-1 / p)(1-3 / p)} \phi^{(k-1)}(T)\right)\left(C T^{\frac{1-a}{2}}\right)^{\frac{1}{1-a}}
\end{aligned}
$$

Escolhendo agora $\varepsilon=\left(C M T^{\frac{1-a}{a p}}\right)^{\frac{1}{a}}$, obtém-se

$$
\left\|\operatorname{rot} w^{(n-1)}\right\|_{L_{p}\left(Q_{T}\right)} \leq C M\left(\left\|u_{0}\right\|_{W_{p}^{2-2 / p}(\Omega)}+\left\|w_{0}\right\|_{W_{p}^{2-2 / p}(\Omega)}+T^{\delta_{1}} \phi^{(k-1)}(T)\right)
$$

A desigualdade (2.34) vale também para o $\operatorname{rot} u^{(k-1)}$.

O caso da limitação para o termo $\left\|\rho^{(k)}\left(u^{(k-1)} \cdot \nabla\right) w^{(k-1)}\right\|_{L_{p}\left(Q_{T}\right)}$ é similar ao argumento para obter (2.33), pois podemos obter

$$
\begin{aligned}
& \left\|\rho^{(k)}\left(u^{(k-1)} \cdot \nabla\right) w^{(k-1)}\right\|_{L_{p}\left(Q_{T}\right)} \\
\leq & M\left\|u^{(k-1)}\right\|_{L_{\infty}\left(Q_{T}\right)} \cdot T^{\frac{1-a}{p}} \cdot\left\|w^{(k-1)}\right\|_{L^{\infty}\left(Q_{T}\right)}^{(1-a)}\left\|w^{(k-1)}\right\|_{W_{p}^{2,1}\left(Q_{T}\right)^{\circ}}^{a}
\end{aligned}
$$

Como

$$
\left\|w^{(k)}\right\|_{L_{\infty}\left(Q_{T}\right)} \leq C\left(\left\|u_{0}\right\|_{W_{P}^{2-2 / p}(\Omega)}+\left\|w_{0}\right\|_{W_{P}^{2-2 / p}(\Omega)}+T^{(1-1 / p)(1-3 / p)} \phi^{(k-1)}(T)\right),
$$


obtém-se

$$
\begin{aligned}
\left\|\rho^{(k)}\left(u^{(k-1)} \cdot \nabla\right) w^{(k-1)}\right\|_{L_{p}\left(Q_{T}\right)} \leq & T^{\frac{1-a}{p}} M C\left(\left(\left\|u_{0}\right\|_{W_{p}^{2-2 / p}(\Omega)}+\left\|w_{0}\right\|\right)_{W_{p}^{2-2 / p}(\Omega)}\right. \\
& \left.+T^{(1-1 / p)(1-3 / p)} \phi^{(k-1)}(T)\right)^{2-a}\left(\phi^{(k-1)}(T)\right)^{a}
\end{aligned}
$$

e então

$$
\left\|\rho^{(k)}\left(u^{(k-1)} \cdot \nabla\right) w^{(k-1)}\right\|_{L_{p}\left(Q_{T}\right)} \leq M C\left(\left\|u_{0}\right\|^{2}+\left\|w_{0}\right\|^{2}+T^{\delta_{1}} \phi^{(k-1)}(T)^{2}\right) .
$$

Finalmente, de (2.26) junto com todas as limitações anteriores, obtém-se

$$
\begin{aligned}
\phi^{(k)}(T) \leq & K_{1}\left(\| \rho \| _ { C ^ { 1 , 1 } , T ) } \left\{\left\|u_{0}\right\|_{W_{p}^{2-2 / p}(\Omega)}+M\|f\|_{L_{p}\left(Q_{T}\right)}\right.\right. \\
& +M C\left(\left\|u_{0}\right\|_{W_{p}^{2-2 / p}(\Omega)}^{2}+\left\|w_{0}\right\|_{W_{p}^{2-2 / p}(\Omega)}^{2}+T^{\delta} \cdot \phi^{(k-1)}(T)^{2}\right) \\
& \left.+2 \mu_{r} M C\left(\left\|u_{0}\right\|_{W_{p}^{2-2 / p}(\Omega)}+\left\|w_{0}\right\|_{W_{p}^{2-2 / p}(\Omega)}+T^{\delta_{1}} \phi^{(k-1)}(T)\right)\right\} \\
& +K_{2}\left(\|\rho\|_{C^{1,1}}, T\right)\left\{\left\|w_{0}\right\|_{W_{p}^{2-2 / p}(\Omega)}+M\|g\|_{L_{p}\left(Q_{T}\right)}\right. \\
& +M C\left(\left\|u_{0}\right\|_{W_{p}^{2-2 / p}(\Omega)}^{2}+\left\|w_{0}\right\|_{W_{p}^{2-2 / p}(\Omega)}^{2}+T^{\delta} \phi^{(k-1)}(T)^{2}\right) \\
& \left.+2 \mu_{r} M C\left(\left\|\left.u_{0}\right|_{W_{p}^{2-2 / p}(\Omega)}+\right\| w_{0} \|_{W_{p}^{2-2 / p}(\Omega)}+T^{\delta_{1}} \phi^{(k-1)}(T)\right)\right\}
\end{aligned}
$$

Finalmente, $\operatorname{com} C=\max \{(1+M C), 2 M C, M\}$, obtemos

$$
\begin{aligned}
\phi^{(k)}(T) \leq & C K\left(\|\rho\|_{C^{1,1}}, T\right)\left\{\left(\left\|u_{0}\right\|_{W_{p}^{2-2 / p}(\Omega)}+\left\|w_{0}\right\|_{W_{p}^{2-2 / p}(\Omega)}\right)\right. \\
& +\left(\|\left(u_{0}\left\|_{W_{p}^{2-2 / p}(\Omega)}^{2}+\right\| w_{0} \|_{W_{p}^{2-2 / p}(\Omega)}^{2}\right)\right. \\
& \left.\left(\|f\|_{L_{p}\left(Q_{T}\right)}+\|g\|_{L_{p}\left(Q_{T}\right)}\right)+T^{\delta_{1}} \phi^{(k-1)}(T)+T^{\delta} \phi^{(k-1)}(T)^{2}\right\}
\end{aligned}
$$

Observemos agora que

$$
\begin{aligned}
\frac{\left|\rho^{(k)}(x, t)-\rho^{(k)}(y, s)\right|}{|x-y|+|t-s|} & =\frac{\left|\rho^{(k)}(x, t)-\rho^{(k)}(y, t)+\rho^{(k)}(y, t)-\rho^{(k)}(y, s)\right|}{|x-y|+|t-s|} \\
& \leq \frac{\left|\rho^{(k)}(x, t)-\rho^{(k)}(y, t)\right|}{|x-y|}+\frac{\left|\rho^{(k)}(y, t)-\rho^{(k)}(y, s)\right|}{|t-s|} .
\end{aligned}
$$

Definamos a aplicação

$$
\varphi(s)=\rho^{(k)}(s y+(1-s) x, t)
$$


com $t$ fixo e $s \in[0,1]$. Portanto,

$$
\begin{aligned}
\left|\rho^{(k)}(y, t)-\rho^{(k)}(x, t)\right| & =|\varphi(1)-\varphi(0)|=\left|\int_{0}^{1} \varphi^{\prime}(s) \mathrm{d} s\right| \\
& \leq \int_{0}^{1}\left|\nabla \rho^{(k)}(s y+(1-s) x, t) \cdot(y-x)\right| \mathrm{d} s \\
& \leq\left\|\nabla \rho^{(k)}\right\|_{L^{\infty}\left(Q_{T}\right)}|y-x| .
\end{aligned}
$$

Além disso, se $t, s \in[0, T]$ qualquer, obtemos

$$
\left|\rho^{(k)}(y, t)-\rho^{(k)}(y, s)\right|=\left|\int_{s}^{t} \rho_{t}^{(k)}(y, \theta) \mathrm{d} \theta\right| \leq\left\|\rho_{t}^{(k)}\right\|_{L^{\infty}\left(Q_{T}\right)}|t-s| .
$$

Usando estas relações concluímos que

$$
\left\|\rho^{(k)}\right\|_{C^{1,1}\left(Q_{T}\right)} \leq M+\left\|\nabla \rho^{(k)}\right\|_{L^{\infty}\left(Q_{T}\right)}+\left\|\rho_{t}^{(k)}\right\|_{L^{\infty}\left(Q_{T}\right)}
$$

Usando as estimativas do Lema 2.3, concluímos que

$$
\begin{aligned}
\left\|\rho^{(k)}\right\|_{C^{1,1}\left(Q_{T}\right) \leq} \leq & M+\sqrt{3}\left(1+\left\|u^{(k-1)}\right\|_{L^{\infty}\left(Q_{T}\right)}\right) \\
& \times\left\|\nabla \rho_{0}\right\|_{L_{\infty}(\Omega)} \exp \left(\int_{0}^{T}\left\|\nabla u^{(k-1)}(t)\right\|_{L^{\infty}(\Omega)} \mathrm{d} t\right) \\
\leq & M+\sqrt{3}\left(1+C_{1}\left(\left\|u_{0}\right\|_{W_{P}^{2-2 / p}(\Omega)}\right.\right. \\
& \left.+T^{(1-1 / p)(1-3 / p)} \phi^{(k-1)}(T)\right)^{2-a}\left(\phi^{(k-1)}(T)\right)^{a}
\end{aligned}
$$

e então

$$
\left\|\rho^{(k)}\left(u^{(k-1)} \cdot \nabla\right) w^{(k-1)}\right\|_{L_{p}\left(Q_{T}\right)} \leq M C\left(\left\|u_{0}\right\|^{2}+\left\|w_{0}\right\|^{2}+T^{\delta_{1}} \phi^{(k-1)}(T)^{2}\right) .
$$

Finalmente, de (2.26) junto com todas as limitações anteriores, obtém-se

$$
\begin{aligned}
\phi^{(k)}(T) \leq & K_{1}\left(\| \rho \| _ { C ^ { 1 , 1 } , T ) } \left\{\left\|u_{0}\right\|_{W_{p}^{2-2 / p}(\Omega)}+M\|f\|_{L_{p}\left(Q_{T}\right)}\right.\right. \\
& +M C\left(\left\|u_{0}\right\|_{W_{p}^{2-2 / p}(\Omega)}^{2}+\left\|w_{0}\right\|_{W_{p}^{2-2 / p}(\Omega)}^{2}+T^{\delta} \cdot \phi^{(k-1)}(T)^{2}\right) \\
& \left.+2 \mu_{r} M C\left(\left\|u_{0}\right\|_{W_{p}^{2-2 / p}(\Omega)}+\left\|w_{0}\right\|_{W_{p}^{2-2 / p}(\Omega)}+T^{\delta_{1}} \phi^{(k-1)}(T)\right)\right\} \\
& +K_{2}\left(\|\rho\|_{C^{1,1}}, T\right)\left\{\left\|w_{0}\right\|_{W_{p}^{2-2 / p}(\Omega)}+M\|g\|_{L_{p}\left(Q_{T}\right)}\right. \\
& +M C\left(\left\|u_{0}\right\|_{W_{p}^{2-2 / p}(\Omega)}^{2}+\left\|w_{0}\right\|_{W_{P}^{2-2 / p}(\Omega)}^{2}+T^{\delta} \phi^{(k-1)}(T)^{2}\right) \\
& \left.+2 \mu_{r} M C\left(\left\|\left.u_{0}\right|_{W_{P}^{2-2 / p}(\Omega)}+\right\| w_{0} \|_{W_{P}^{2-2 / p}(\Omega)}+T^{\delta_{1}} \phi^{(k-1)}(T)\right)\right\}
\end{aligned}
$$


Finalmente, com $C=\max \{(1+M C), 2 M C, M\}$, obtemos

$$
\begin{aligned}
\phi^{(k)}(T) \leq & C K\left(\|\rho\|_{C^{1,1}}, T\right)\left\{\left(\left\|u_{0}\right\|_{W_{p}^{2-2 / p}(\Omega)}+\left\|w_{0}\right\|_{W_{P}^{2-2 / p}(\Omega)}\right)\right. \\
& +\left(\|\left(u_{0}\left\|_{W_{P}^{2-2 / p}(\Omega)}^{2}+\right\| w_{0} \|_{W_{p}^{2-2 / p}(\Omega)}^{2}\right)\right. \\
& \left.\left(\|f\|_{L_{p}\left(Q_{T}\right)}+\|g\|_{L_{p}\left(Q_{T}\right)}\right)+T^{\delta_{1}} \phi^{(k-1)}(T)+T^{\delta} \phi^{(k-1)}(T)^{2}\right\}
\end{aligned}
$$

Observemos agora que

$$
\begin{aligned}
\frac{\left|\rho^{(k)}(x, t)-\rho^{(k)}(y, s)\right|}{|x-y|+|t-s|} & =\frac{\left|\rho^{(k)}(x, t)-\rho^{(k)}(y, t)+\rho^{(k)}(y, t)-\rho^{(k)}(y, s)\right|}{|x-y|+|t-s|} \\
& \leq \frac{\left|\rho^{(k)}(x, t)-\rho^{(k)}(y, t)\right|}{|x-y|}+\frac{\left|\rho^{(k)}(y, t)-\rho^{(k)}(y, s)\right|}{|t-s|} .
\end{aligned}
$$

Definamos a aplicação

$$
\varphi(s)=\rho^{(k)}(s y+(1-s) x, t)
$$

com $t$ fixo e $s \in[0,1]$. Portanto,

$$
\begin{aligned}
\left|\rho^{(k)}(y, t)-\rho^{(k)}(x, t)\right| & =|\varphi(1)-\varphi(0)|=\left|\int_{0}^{1} \varphi^{\prime}(s) \mathrm{d} s\right| \\
& \leq \int_{0}^{1}\left|\nabla \rho^{(k)}(s y+(1-s) x, t) \cdot(y-x)\right| \mathrm{d} s \\
& \leq\left\|\nabla \rho^{(k)}\right\|_{L^{\infty}\left(Q_{T}\right)}|y-x| .
\end{aligned}
$$

Além disso, se $t, s \in[0, T]$ qualquer, obtemos

$$
\left|\rho^{(k)}(y, t)-\rho^{(k)}(y, s)\right|=\left|\int_{s}^{t} \rho_{t}^{(k)}(y, \theta) \mathrm{d} \theta\right| \leq\left\|\rho_{t}^{(k)}\right\|_{L^{\infty}\left(Q_{T}\right)}|t-s| .
$$

Usando estas relações concluímos que

$$
\left\|\rho^{(k)}\right\|_{C^{1,1}\left(Q_{T}\right)} \leq M+\left\|\nabla \rho^{(k)}\right\|_{L^{\infty}\left(Q_{T}\right)}+\left\|\rho_{t}^{(k)}\right\|_{L^{\infty}\left(Q_{T}\right)} .
$$

Usando as estimativas do Lema 2.3, concluímos que

$\left\|\rho^{(k)}\right\|_{C^{1,1}\left(Q_{T}\right)} \leq M+\sqrt{3}\left(1+\left\|u^{(k-1)}\right\|_{L^{\infty}\left(Q_{T}\right)}\right)$ 


$$
\begin{aligned}
& \times\left\|\nabla \rho_{0}\right\|_{L_{\infty}(\Omega)} \exp \left(\int_{0}^{T}\left\|\nabla u^{(k-1)}(t)\right\|_{L^{\infty}(\Omega)} \mathrm{d} t\right) \\
\leq & M+\sqrt{3}\left(1+C_{1}\left(\left\|u_{0}\right\|_{W_{p}^{2-2 / p}(\Omega)}+\left\|w_{0}\right\|_{W_{P}^{2-2 / p}(\Omega)}+T^{(1-1 / p)(1-3 / p)} \phi^{(k-1)}(T)\right)\right. \\
& \times\left\|\nabla \rho_{0}\right\|_{L_{\infty}(\Omega)} \exp \left(C_{5} T^{(1-1 / p)(1-3 / p)} \phi^{(k-1)}(T)\right) \\
\equiv & K_{3}\left(\phi^{(k-1)}(T), T\right) .
\end{aligned}
$$

Conseqüentemente, temos

$$
\begin{aligned}
\phi^{(k)}(T) \leq & K\left(K_{3}\left(\phi^{(k-1)}(T), T\right), T\right) \times\left\{\left(\left\|u_{0}\right\|_{W_{p}^{2-2 / p}(\Omega)}+\left\|w_{0}\right\|_{W_{p}^{2-2 / p}(\Omega)}\right.\right. \\
& +\left(\left\|u_{0}\right\|_{W_{p}^{2-2 / p}(\Omega)}^{2}+\left\|w_{0}\right\|_{W_{p}^{2-2 / p}(\Omega)}^{2}+\|f\|_{L_{p}\left(Q_{T}\right)}+\|g\|_{L_{p}\left(Q_{T}\right)}\right. \\
& \left.+T^{\delta_{1}} \phi^{(k-1)}(T)+T^{\delta} \phi^{(k-1)}(T)^{2}\right\} .
\end{aligned}
$$

Para terminar a prova do Lema 2.7, queremos escolher as constantes $A_{1}$ e $T_{1}$ tais que

$$
\phi^{(k-1)}\left(T_{1}\right) \leq A_{1} \Rightarrow \phi^{(k)}\left(T_{1}\right) \leq A_{1}
$$

Notemos que se escolhermos $A_{1}$ tal que

$$
\begin{aligned}
A_{1} \geq & K\left(M+\sqrt{3}\left\{1+C_{1}\left(\left\|u_{0}\right\|_{W_{p}^{2-2 / p}(\Omega)}+\left\|w_{0}\right\|_{W_{p}^{2-2 / p}(\Omega)}\right)+1\right\}\left\|\nabla \rho_{0}\right\|_{L_{\infty}(\Omega)}, T\right) \\
& \times\left(\left\|u_{0}\right\|_{W_{p}^{2-2 / p}(\Omega)}+\left\|w_{0}\right\|_{W_{p}^{2-2 / p}(\Omega)}+\left\|u_{0}\right\|_{W_{p}^{2-2 / p}(\Omega)}^{2}+\left\|w_{0}\right\|_{W_{p}^{2-2 / p}(\Omega)}^{2}\right. \\
& \left.+\|f\|_{L_{p}\left(Q_{T}\right)}+\|g\|_{L_{p}\left(Q_{T}\right)}+2\right)
\end{aligned}
$$

e definimos

$$
T_{1}=\min \left\{A_{1}^{-1(1-1 / p)^{-1}(1-3 / p)^{-1}}, A_{1}^{-2 / \delta}, A_{1}^{-1 / \delta_{1}},\left(C_{1 S} A_{1}\right)^{-(1-1 / p)^{-1}}\right\}
$$

Logo, finalizamos a prova do Lema 2.7, uma vez que se verifica por cálculo direto que

$$
\begin{aligned}
\phi^{(1)}\left(T_{1}\right) \leq & K\left(M+\sqrt{3}\left\|\nabla \rho_{0}\right\|_{L_{\infty}(\Omega)}, T_{1}\right)\left\{\left\|u_{0}\right\|_{W_{p}^{2-2 / p}(\Omega)}\right. \\
& \left.+\left\|w_{0}\right\|_{W_{p}^{2-2 / p}(\Omega)}+M\left(\|f\|_{L_{P}\left(Q_{T}\right)}+\|g\|_{L_{p}\left(Q_{T}\right)}\right)\right\} \\
\leq & A_{1} .
\end{aligned}
$$




\subsection{Demonstração do teorema principal}

Definamos as seqüências

$$
\begin{aligned}
\rho^{(n, k)} & =\rho^{(n+k)}-\rho^{(n)}, \\
u^{(n, k)} & =u^{(n+k)}-u^{(n)}, \\
w^{(n, k)} & =w^{(n+k)}-w^{(n)}, \\
p^{(n, k)} & =p^{(n+k)}-p^{(n)} .
\end{aligned}
$$

Temos que estas seqüências satisfazem os problemas seguintes:

$$
\begin{aligned}
& \left\{\begin{array}{l}
\frac{\partial \rho^{(n, k)}}{\partial t}+\left(u^{(n+k-1)} \cdot \nabla\right) \rho^{(n, k)}=-\left(u^{(n-1, k)} \cdot \nabla\right) \rho^{(n)} \\
\rho^{(n, k)}(0)=0
\end{array}\right. \\
& \left\{\begin{array}{l}
\rho^{(n+k)} \frac{\partial u^{(n, k)}}{\partial t}-\left(\mu+\mu_{r}\right) \Delta u^{(n, k)}+\nabla p^{(n, k)}=F^{(n, k)} \\
\operatorname{div} u^{(n, k)}=0 \\
\left.u^{(n, k)}\right|_{\Sigma_{T}}=0 \\
u^{(n, k)}(0)=0
\end{array}\right.
\end{aligned}
$$

onde

$$
\begin{aligned}
F^{(n, k)}= & 2 \mu_{r} \operatorname{rot} w^{(n-1, p)}-\rho^{(n, k)}\left[f-u_{t}^{(n)}-\left(u^{(n-1)} \cdot \nabla\right) u^{(n-1)}\right] \\
& \left.-\rho^{(n+k)}\left[u^{(n-1, k)} \cdot \nabla\right) u^{(n-1+k)}-\left(u^{(n-1)} \cdot \nabla\right) u^{(n-1, k)}\right]
\end{aligned}
$$

e finalmente

$$
\left\{\begin{array}{l}
\rho^{(n+k)} \frac{\partial w^{(n, k)}}{\partial t}-\nu \Delta w^{(n, k)}-\gamma \nabla \operatorname{div} w^{(n, k)}+4 \mu_{r} w^{(n, k)}=G^{(n, k)} \\
\left.w^{(n, k)}\right|_{\Sigma_{T}}=0 \\
w^{(n, k)}(0)=0
\end{array}\right.
$$

onde

$$
\begin{aligned}
G^{n, k}= & 2 \mu_{r} \operatorname{rot} w^{(n-1, k)}-\rho^{(n, k)}\left[g-w_{t}^{(n)}-\left(u^{(n-1)} \cdot \nabla\right) w^{(n-1)}\right. \\
& -\rho^{(n+k)}\left[\left(u^{(n-1+k)} \cdot \nabla\right) w^{(n-1+k)}-\left(u^{(n-1, k)} \cdot \nabla\right) w^{(n-1)}\right] .
\end{aligned}
$$


Definamos a função

$$
\psi^{(n, k)}(t)=\left\|u^{(n, k)}\right\|_{W_{P}^{2,1}\left(Q_{T}\right)}+\left\|w^{(n, k)}\right\|_{W_{P}^{2,1}\left(Q_{T}\right)}+\left\|\nabla \rho^{(n, k)}\right\|_{L_{P}\left(Q_{T}\right)} .
$$

Então, para todo $t \in\left(0, T_{1}\right]$

$$
\begin{aligned}
& \left\|F^{(n, k)}\right\|_{L_{p}\left(Q_{t}\right)} \leq C\left(\left\|\nabla w^{(n-1, k)}\right\|_{L_{p}\left(Q_{t}\right)}+\left\|\rho^{(n, k)}\right\|_{L^{\infty}\left(Q_{t}\right)}\left\{\|f\|_{L_{p}\left(Q_{t}\right)}\right.\right. \\
& \left.+\left\|u_{t}^{(n)}\right\|_{L_{p}\left(Q_{t}\right)}\left\|\left(u^{n-1} \cdot \nabla\right) u^{(n-1)}\right\|_{L_{p}\left(Q_{t}\right)}\right\} \\
& +\left\|\rho^{(n+k)}\right\|_{L^{\infty}\left(Q_{t}\right)}\left\{\left\|\left(u^{(n-1, k)} \cdot \nabla\right) u^{(n-1+k)}\right\|_{L_{p}\left(Q_{t}\right)}\right. \\
& +\left\|\left(u^{(n-1)} \cdot \nabla\right) u^{(n-1, k)}\right\|_{L_{p}\left(Q_{t}\right)} .
\end{aligned}
$$

Daqui em diante passamos a limitar todos os termos dos lados direitos das equações (2.39) e (2.40).

Observemos que

$$
\begin{aligned}
& \left\|\rho^{(n, k)}\left[f-u_{t}^{n}-\left(u^{(n-1)} \cdot \nabla\right) u^{n-1}\right]\right\|_{L_{p}\left(Q_{t}\right)}^{p} \\
\leq & \left\|\rho^{(n, k)}\right\|_{L_{\infty}\left(Q_{t}\right)}\left(\|f\|_{L_{p}\left(Q_{t}\right)}^{p}+\left\|u_{t}^{n}\right\|_{L_{p}\left(Q_{t}\right)}^{p}+\left\|u^{(n-1)}\right\|_{L_{\infty}\left(Q_{t}\right)}^{p}\left\|\nabla u^{(n-1)}\right\|_{L_{p}\left(Q_{t}\right)}^{p}\right) \\
\leq & \left\|\rho^{(n, k)}\right\|_{L_{\infty}\left(Q_{t}\right)}\left[A_{1}^{p}+C\left(\left\|u_{0}\right\|_{W_{p}^{2-1 / p}(\Omega)}+\left\|w_{0}\right\|_{W_{p}^{2-2 / p}(\Omega)}+1\right) A_{1}^{p}+\|f\|_{L_{p}\left(Q_{t}\right)}\right] .
\end{aligned}
$$

Portanto,

$$
\left\|\rho^{(n, k)}\left[f-u_{t}^{n}-\left(u^{(n-1)} \cdot \nabla\right) u^{(n-1)}\right]\right\|_{L_{p}\left(Q_{t}\right)}^{p} \leq C_{1}\left\|\rho^{(n, k)}\right\|_{L_{\infty}\left(Q_{t}\right)} .
$$

Por outro lado, temos

$$
\begin{aligned}
& \| \rho^{(n+k)}\left[\left(u^{(n-1, k)} \cdot \nabla\right) u^{(n-1+k)}-\left(u^{(n-1)} \cdot \nabla\right) u^{(n-1, k)} \|_{L_{p}\left(Q_{T}\right)}^{p}\right] \\
\leq & M^{p} \int_{0}^{t} \mathrm{~d} \tau \int_{\Omega}\left|\nabla u^{(n-1+k)}\right|^{p}\left|u^{(n-1, k)}\right|^{p}+\left.\left|u^{(n-1)}\right|\right|^{p}\left|\nabla u^{(n-1, k)}\right|^{p} \mathrm{~d} x \\
\leq & M^{p}\left(\sup _{0 \leq \Delta \leq t}\left\|\nabla u^{(n-1+k)}\right\|_{L_{p}(\Omega)}^{p} \int_{0}^{t}\left\|u^{(n-1, k)}\right\|^{p} \mathrm{~d} \tau\right. \\
& \left.+\left\|u^{(n-1)}\right\|_{L_{\infty}\left(Q_{T}\right)}^{p} \int_{0}^{t}\left\|\nabla u^{(n-1, k)}\right\|_{L_{p}(\Omega)}^{p} \mathrm{~d} \tau\right) \\
\leq & C M^{p}\left\{\left\|u^{(n-1+k)}\right\|_{W_{p}^{2,1}\left(Q_{T}\right)} \int_{0}^{t}\left\|u^{(n-1, k)}\right\|_{W_{p}^{2,1}\left(Q_{\tau}\right)} \mathrm{d} \tau\right.
\end{aligned}
$$




$$
\begin{aligned}
& +\left(1+\left\|u_{0}\right\|_{W_{P}^{2-2 / p}(\Omega)}+\left\|w_{0}\right\|_{W_{P}^{2-2 / p}(\Omega)}\right) \\
& \left.\int_{0}^{t}\left\|u^{(n-1, k)}\right\|_{W_{P}^{2,1}\left(Q_{\tau}\right)} \mathrm{d} \tau\right\}
\end{aligned}
$$

Portanto, obtém-se

$$
\| \rho^{(n+k)}\left[\left(u^{(n-1, k)} \cdot \nabla\right) u^{(n-1+k)}-\left(u^{(n-1)} \cdot \nabla\right) u^{(n-1, k)} \|_{L_{p}\left(Q_{t}\right)}^{p} \leq C_{2} \int_{0}^{t} \psi^{(n-1, k)}(\tau) \mathrm{d} \tau\right.
$$

Observemos que

$$
\begin{aligned}
\left\|\nabla w^{(n-1, k)}\right\|_{L_{p}\left(Q_{t}\right)}^{p} & \leq \int_{0}^{t}\left\|\nabla w^{(n-1, k)}(\tau)\right\|_{L_{p}(\Omega)}^{p} \mathrm{~d} t \\
& \leq \int_{0}^{t}\left\|w^{(n-1, k)}(\tau)\right\|_{W_{p}^{1}(\Omega)}^{p} \mathrm{~d} \tau \\
& \leq C \int_{0}^{t}\left\|w^{(n-1, k)}(\tau)\right\|_{W_{p}^{2,1}\left(Q_{\tau}\right)}^{p} \mathrm{~d} \tau .
\end{aligned}
$$

Logo,

$$
\left\|\nabla w^{(n-1, k)}\right\|_{L_{p}\left(Q_{t}\right)}^{p} \leq C \int_{0}^{t} \psi^{(n-1, k)}(\tau) \mathrm{d} \tau
$$

Por outro lado,

$$
\begin{aligned}
& \left\|\left(u^{(n-1, k)} \cdot \nabla\right) u^{(n-1+k)}\right\|_{L_{p}\left(Q_{t}\right)}^{p} \leq \int_{0}^{t}\left\|\nabla u^{(n-1+k)}\right\|_{L_{p}(\Omega)}^{p}\left\|u^{(n-1, k)}\right\|_{L^{\infty}(\Omega)}^{p} \mathrm{~d} \tau \\
& \leq \sup _{0 \leq \tau \leq t}\left\|\nabla u^{(n-1+k)}\right\|_{L_{p}(\Omega)}^{p} \int_{0}^{t}\left\|u^{(n-1, k)}\right\|_{L_{\infty}(\Omega)}^{p} \mathrm{~d} \tau \\
& \leq \sup _{0 \leq \tau \leq t}\left\|u^{(n-1+k)}\right\|_{W_{p}^{1}(\Omega)}^{p} \int_{0}^{t}\left\|u^{(n-1, k)}(\tau)\right\|_{W_{P}^{1}(\Omega)}^{p} \mathrm{~d} \tau \\
& \leq \sup _{0 \leq s \leq t}\left\|u^{(n-1+k)}(\tau)\right\|_{W_{p}^{2-2 / p}(\Omega)}^{p} \int_{0}^{t}\left\|u^{(n-1, k)}(\tau)\right\|_{W_{p}^{2-2 / p}(\Omega)}^{p} \mathrm{~d} \tau \\
& \leq\left(\left\|u^{(n-1+k)}(0)\right\|_{W_{P}^{2-2 / p}(\Omega)}+\hat{C}\left\|u^{(n-1+k)}(\tau)\right\|_{W_{P}^{2,1}\left(Q_{t}\right)}\right)^{p} \\
& \quad \int_{0}^{t} \hat{C}^{p}\left\|u^{(n-1, k)}\right\|_{W_{p}^{2,1}\left(Q_{\tau}\right)}^{p} \mathrm{~d} \tau \\
& \left\|\left(u^{(n-1, k)} \cdot \nabla\right) u^{(n-1+k)}\right\|_{L_{p}\left(Q_{t}\right)}^{p} \leq C \int_{0}^{t} \psi^{(n-1, k)}(\tau)^{p} \mathrm{~d} \tau
\end{aligned}
$$

$\mathrm{e}$

$$
\left\|\left(u^{n-1} \cdot \nabla\right) u^{(n-1, k)}\right\|_{L_{p}\left(Q_{t}\right)}^{p} \leq \int_{0}^{t} \mathrm{~d} \tau \int_{\Omega}\left|u^{(n-1)}\right|^{p}\left|\nabla u^{(n-1, k)}\right|^{p} \mathrm{~d} x
$$




$$
\begin{aligned}
& \leq\left\|u^{(n-1)}\right\|_{L_{\infty}\left(Q_{t}\right)}^{p} \int_{0}^{t}\left\|\nabla u^{(n-1, k)}\right\|_{L_{p}(\Omega)}^{p} \mathrm{~d} \tau \\
& \leq \sup _{0 \leq \tau \leq t}\left\|u^{(n-1)}\right\|_{W_{p}^{2-2 / p}(\Omega)}^{p} \int_{0}^{t}\left\|u^{(n-1, k)}\right\|_{W_{p}^{1}(\Omega)}^{p} \mathrm{~d} \tau \\
& \leq \sup _{0 \leq \tau \leq t}\left\|u^{(n-1)}\right\|_{W_{p}^{2-2 / p}(\Omega)} \int_{0}^{t}\left\|u^{(n-1, k)}\right\|_{W_{p}^{2-2 / p}(\Omega)}^{p} \mathrm{~d} \tau \\
& \leq\left(\left\|u^{(n-1)}(0)\right\|_{W^{2-2 / p}(\Omega)}+\hat{C}\left\|u^{(n-1)}\right\|_{W_{p}^{2,1}\left(Q_{t}\right)}\right)^{p} \\
& \hat{C}^{p} \int_{0}^{t}\left\|u^{(n-1, k)}\right\|_{W_{p}^{2,1}\left(Q_{t}\right)}^{p} \mathrm{~d} \tau \\
&\left\|\left(u^{(n-1)} \cdot \nabla\right) u^{(n-1, k)}\right\|_{L_{p}\left(Q_{T}\right)}^{p} \leq C \int_{0}^{t} \psi^{(n-1, k)}(\tau)^{p} \mathrm{~d} \tau .
\end{aligned}
$$

Do Lema 2.4, temos

$$
\begin{aligned}
\left\|\rho^{(n, k)}(t)\right\|_{L_{\infty}(\Omega)} & \leq \int_{0}^{t}\left\|u^{(n-1, k)}(\tau)\right\|_{L_{\infty}(\Omega)} \nabla \rho^{(n)}(\tau) \|_{L_{\infty}(\Omega)} \mathrm{d} \tau \\
& \leq C \int_{0}^{t}\left\|u^{(n-1, k)}\right\|_{W_{p}^{2,1}\left(Q_{\tau}\right)} \mathrm{d} \tau \\
& \leq C \int_{0}^{t} \psi^{(n-1, k)}(\tau) \mathrm{d} \tau
\end{aligned}
$$

Devido à limitação (2.46), da desigualdade (2.41), temos finalmente

$$
\left\|\rho^{(n, k)}\left[f-u_{t}^{n}-\left(u^{(n-1)} \cdot \nabla\right) u^{(n-1)}\right]\right\|_{L\left(Q_{T}\right)} \leq C_{1}\left(\int_{0}^{t} \psi^{(n-1, k)}(\tau) \mathrm{d} \tau\right)^{1 / p}
$$

Conseqüentemente, juntando todas as desigualdades (2.42) - (2.47), obtém-se

$$
\begin{aligned}
\left\|F^{(n, k)}\right\| & \leq \bar{C}_{1} \int_{0}^{t} \psi^{(n-1)}(\tau) \mathrm{d} \tau+\bar{C}_{2}\left(\int_{0}^{t} \psi^{(n-1, k)}(\tau)^{p} \mathrm{~d} \tau\right)^{1 / p} \\
& \leq C\left(\int_{0}^{t} \psi^{(n-1, k)}(\tau)^{p} \mathrm{~d} \tau\right)^{1 / p}
\end{aligned}
$$

Um cálculo similar para estimar os termos de $G^{(n, k)}$ resulta em

$$
\left\|G^{(n, k)}\right\|_{L_{p}\left(Q_{T}\right)} \leq \bar{C}\left(\int_{0}^{t} \psi^{(n-1, k)}(\tau)^{p} \mathrm{~d} \tau\right)^{p}
$$

Usando o Lema 2.2, temos que

$$
\left\|u^{(n, k)}\right\|_{W_{P}^{2,1}\left(Q_{T}\right)}+\left\|\nabla p^{(n, k)}\right\|_{L_{p}\left(Q_{T}\right)} \leq \bar{K}_{1}\left\|F^{(n, k)}\right\|_{L_{P}\left(Q_{T}\right)}
$$


e do Lema 2.5, temos

$$
\left\|w^{(n-1, k)}\right\|_{W_{P}^{2,1}\left(Q_{T}\right)} \leq \bar{K}_{2}\left\|G^{(n, k)}\right\|_{L_{P}\left(Q_{T}\right)}
$$

Agora, somando (2.48) e (2.49) e levando em conta as limitações acima para $\left\|F^{(n, k)}\right\|_{L_{p}\left(Q_{T}\right)}$ e de $\left\|G^{(n, k)}\right\|_{L_{p}\left(Q_{T}\right)}$, obtém-se finalmente para $t \in\left[0, T_{1}\right]$ e $p>3$,

$$
\left[\psi^{(n, k)}(t)\right]^{p} \leq C^{p} \int_{0}^{t}\left[\psi^{(n-1, k)}(\tau)\right]^{p} \mathrm{~d} \tau
$$

e, conseqüentemente, temos que $\psi^{(n, k)}(t) \mapsto 0$ quando $n, k \rightarrow \infty$ para todo $t \in\left[0, T_{1}\right]$, já que

$$
\begin{aligned}
\alpha_{n} & =\left[\psi^{(n, k)}(t)\right]^{p} \leq \int_{0}^{t} \alpha_{n-1}\left(\tau_{1}\right) \mathrm{d} \tau_{1} \\
& \leq C \int_{0}^{t} \int_{0}^{t_{1}} \alpha_{n-2}\left(\tau_{2}\right) \mathrm{d} \tau_{2} \mathrm{~d} \tau_{1} \\
& \leq C^{(n-k-1)} \int_{0}^{t} \int_{0}^{t_{1}} \int_{0}^{t_{2}} \cdots \int_{0}^{t_{n-k}} \alpha_{1}\left(\tau_{n}\right) \mathrm{d} \tau_{(n-k)} \cdots \mathrm{d} \tau_{1} \\
& \leq \frac{C^{(n-k-1)} F_{1}^{(n-1+k) / p}}{((n+k-1) !)^{p}} .
\end{aligned}
$$

Portanto, temos que

$$
\psi^{(n, k)}\left(T_{1}\right) \leq \frac{C^{(n-k-2)} T_{1}^{(n+k-1) / p}}{[(n+k-1) !]^{p}} \psi^{(1)}\left(T_{1}\right)=\frac{C T_{1}^{(n+k-1) / p} \psi^{(1)}\left(T_{1}\right)}{\Gamma(n+k)^{p}} .
$$

Logo,

$$
\sum_{k=1}^{\infty} \psi^{(n, k)}\left(T_{1}\right)<+\infty
$$

Assim, concluímos que a seqüência $\left(u^{(n)}, w^{(n)}, p^{(n)}\right)(x, t)$ convergem a uma solução $(u, w, p)$ quando $n \rightarrow+\infty$. Como temos convergência forte em $W_{p}^{2,1}\left(Q_{T_{1}}\right)$, $(u, w, p)$ satisfazem suas respectivas equações.

Agora, devido a que

$$
\left\|\rho^{(n, k)}\right\|_{L_{\infty}(\Omega)} \leq\left\|\rho^{(n, k)}\right\|_{W_{p}^{1}(\Omega)} \leq C \int_{0}^{t}\left\|u^{(n+k-1)}(\tau)\right\|_{W_{p}^{1}(\Omega)} \mathrm{d} \tau
$$


temos que $\rho^{n} \mapsto \rho$ em $W_{p}^{1}(\Omega)$ e daqui que $\rho^{n} \mapsto \rho$ em $L_{\infty}(\Omega)$. Por outro lado, temos que

$$
\sup _{0 \leq t \leq T_{1}}\left\|\nabla \rho^{(n)}(t)\right\|_{W_{p}^{1}(\Omega)} \leq\left\|\nabla \rho_{0}\right\| \exp \left(C_{*}\left(T_{1}\right)^{\frac{p-1}{p}}=C_{3} .\right.
$$

Disto vem que $\nabla \rho^{n} \in C\left(\left[0, T_{1}\right] ; W_{p}^{1}(\Omega)\right)$. Como

$$
\sup _{0 \leq t \leq T_{1}}\left\|\nabla \rho^{(n)}-\nabla \rho\right\|_{W_{p}^{1}(\Omega)} \mapsto 0,
$$

quando $n$ tende para o infinito e, portanto, temos que $\nabla \rho^{(n)}$ converge para $\nabla \rho$ em $C\left(\left[0, T_{1}\right] ; W_{p}^{1}(\Omega)\right)$ quando $n \mapsto \infty$. Notemos, ainda, que pela imersão de Sobolev para $p>3$, temos

$$
\sup _{0 \leq t \leq T_{1}}\left\|\nabla \rho^{(n)}-\nabla \rho\right\|_{L_{\infty}(\Omega)} \leq \sup _{0 \leq t \leq T_{1}}\left\|\nabla \rho^{(n)}-\nabla \rho\right\|_{W_{p}^{1}(\Omega)}
$$

Portanto, temos convergência de $\nabla \rho^{n}$ a $\nabla \rho$ em $C\left(\left[0, T_{1}\right] ; L_{\infty}(\Omega)\right)$. Como

$$
\left\|\nabla \rho^{(n)}\right\|_{L_{\infty}\left(Q_{T_{1}}\right)}+\left\|\rho_{t}^{(n)}\right\|_{L^{\infty}\left(Q_{T_{1}}\right)} \leq K_{3}\left(A_{1}, T_{1}\right)=\bar{C}
$$

então obtemos que $\rho^{(n)} \in W^{1, \infty}\left(Q_{T_{1}}\right)$, logo temos que $\rho^{(n)} \mapsto \rho \in C\left(\bar{Q}_{T_{1}}\right)$ quando $n$ tende para o infinito.

Já que $\left\{\rho^{(n)}\right\}$ converge só em $C\left(\bar{Q}_{T_{1}}\right)$, não podemos tomar limite diretamente na equação (2.23). Para isto usamos o seguinte argumento: sabemos que existe $\rho \in C\left(\bar{Q}_{T_{1}}\right)$ e $u \in W_{p}^{2,1}\left(Q_{T_{1}}\right)$ tal que

$$
\rho^{(n)} \rightarrow \rho \text { quando } n \mapsto \infty \text { uniformemente sobre } Q_{T_{1}}
$$

e

$$
\left\|u^{(n)}-u\right\|_{W_{P}^{2,1}\left(Q_{T_{1}}\right)} \mapsto 0 \text { quando } n \mapsto \infty .
$$

Claramente, $\rho(x, 0)=\rho_{0}(x)$.

Multiplicando a equação (2.23) por uma função suave $\psi(x, t)$ e integrando sobre $Q_{T_{1}}$, obtém-se

$$
0=\int_{\Omega} \varphi_{0}(x) \psi(x, 0)+\int_{0}^{T_{1}} \int_{\Omega} \rho^{(n)}(x, t)\left\{\frac{\partial \psi}{\partial t}(x, t)+u^{(n-1)} \cdot \nabla \psi\right\} \mathrm{d} x \mathrm{~d} t,
$$


pois $\rho_{n}(x, 0)=\rho_{0}(x), \operatorname{div} u^{n-1}=0$ e $\psi\left(x, T_{1}\right)=0$. Quando $n$ tende para o infinito, vem que

$$
0=\int_{\Omega} \rho_{0}(x) \psi(x, 0)+\int_{0}^{T_{1}} \int_{\Omega} \rho(x, t)\left\{\frac{\partial \psi}{\partial t}+u \cdot \nabla \psi(x, t)\right\} \mathrm{d} x \mathrm{~d} t
$$

Usando o resultado abaixo, provamos que efetivamente $\rho(x, t)$ é solução da equação de densidade.

Lema 2.8 (Ladyzhenskaya-Solonnikov [41]) Seja $\rho_{0}(x) \in W^{1, \infty}(\Omega), \rho \in L^{1}\left(\left(0, T_{1}\right) \times\right.$ $\Omega)$ e $v \in C\left(\bar{Q}_{T_{1}}\right)$ satisfazendo

$$
\int_{0}^{T_{1}}\|\nabla v(t)\|_{L_{\infty}} \mathrm{d} t<+\infty
$$

e, além disso,

$$
0=\int_{\Omega} \rho_{0}(x) \psi(x, 0)+\int_{0}^{T_{1}} \int_{\Omega}(x, t)\left\{\frac{\partial \psi}{\partial t}+v \cdot \nabla \psi(x, t)\right\} \mathrm{d} x \mathrm{~d} t
$$

para toda $\psi$ suave, tal que $\psi\left(T_{1}, x\right)=0$, então $\rho \in W^{1, \infty}\left(\left(0, T_{1}\right) \times \Omega\right)$ e satisfaz

$$
\frac{\partial \rho}{\partial t}+v \cdot \nabla \rho=0, \quad \rho(x, 0)=\rho_{0}(x)
$$

Agora passamos a provar a unicidade da solução.

Para provar a unicidade, suponhamos que $\left(u_{1}, w_{1}, \rho_{1}, p_{1}\right)(x, t)$ e $\left(u_{2}, w_{2}, \rho_{2}, p_{2}\right)(x, t)$ são duas soluções do sistema de equações $(0.1)-(0.7)$. Então as diferenças $U=$ $u_{1}-u_{2}, W=w_{1}-w_{2}, \eta=\rho_{1}-\rho_{2}$ e $P=p_{1}-p_{2}$ satisfaz o sistema abaixo.

$$
\begin{aligned}
& \rho_{1}\left(\frac{\partial U}{\partial t}+\left(u_{1} \cdot \nabla\right) U+(U \cdot \nabla) u_{2}\right)+\eta\left(\frac{\partial u_{2}}{\partial t}+\left(u_{2} \cdot \nabla\right) u_{2}-f\right) \\
& -\left(\mu+\mu_{r}\right) \Delta U+\nabla P=2 \mu_{r} \operatorname{rot}(W) \\
& \rho_{1}\left(\frac{\partial W}{\partial t}+\left(u_{1} \cdot \nabla\right) W+(U \cdot \nabla) w_{2}\right)+\eta\left(\frac{\partial w_{2}}{\partial t}+\left(u_{2} \cdot \nabla\right) w_{2}-g\right) \\
& +\Delta W+2 \mu_{r} W=2 \mu_{r} \operatorname{rot}(U)
\end{aligned}
$$




$$
\begin{aligned}
& \frac{\partial \eta}{\partial t}+\left(u_{1} \cdot \nabla\right) \eta+(U \cdot \nabla) \rho_{2}=0 \\
& U(x, 0)=W(x, 0)=0,\left.\quad U\right|_{\Sigma_{T}}=\left.W\right|_{\Sigma}=0, \quad \eta(x, 0)=0,
\end{aligned}
$$

onde $A W=-\nu \Delta W+\gamma \nabla \operatorname{div} W$.

Observe-se que tomando o produto interno na equação (2.52) com $U$ e integrando sobre o domínio $\Omega$, obtém-se

$$
\begin{aligned}
& \int_{\Omega} \rho_{1}\left(\frac{\partial U}{\partial t}+\left(u_{1} \cdot \nabla\right) U\right) U \mathrm{~d} x=\frac{1}{2} \frac{\mathrm{d}}{\mathrm{d} t} \int_{\Omega} \rho_{1} U^{2} \mathrm{~d} x \\
& \int_{\Omega} \eta\left(\frac{\partial u_{2}}{\partial t}+\left(u_{2} \cdot \nabla\right) u_{2}-f\right) \mathrm{d} x=\int_{\Omega} \eta v U \mathrm{~d} x
\end{aligned}
$$

A função $v=\left(\frac{\partial u_{2}}{\partial t}+\left(u_{2} \cdot \nabla\right) u_{2}-f\right)$ é uma função que pertence a $L_{p}\left(Q_{T}\right)$. Portanto, temos

$$
\begin{aligned}
\left|\int_{\Omega} v U \mathrm{~d} x\right| & \leq\|v\|_{L^{p}(\Omega)^{3}}\|\eta\|_{L^{2}(\Omega)}\|U\|_{L^{\frac{2 p}{p-2}}(\Omega)^{3}} \\
& \leq C(N, p)\|v\|_{L^{p}(\Omega)}\|\eta\|_{L^{2}(\Omega)}\|\nabla U\|_{L^{2}(\Omega)^{9}}^{\frac{N}{p}}\|U\|_{L^{2}(\Omega)^{3}}^{1-\frac{N}{p}} \\
& \leq \varepsilon\|\nabla U\|_{L^{2}(\Omega)}^{2}+C_{3} \varepsilon^{-\frac{N}{2 p-N}}\|v\|_{L^{p}(\Omega)}^{\frac{2 p}{2 p-N}}\left(\left\|\eta_{L^{2}(\Omega)}^{2}+\right\| U \|_{L^{2}(\Omega)^{3}}^{2}\right)
\end{aligned}
$$

Para obter a desigualdade (2.57) usamos os seguintes fatos: para toda $u \in \stackrel{\circ}{W}_{p}^{1}(\Omega)$ tem-se

$$
\|u\|_{L^{\frac{2 p}{p-2}(\Omega)}} \leq C(N, p)\|\nabla u\|_{L^{2}(\Omega)^{9}}^{\frac{N}{p}}\|u\|_{L^{2}(\Omega)^{3}}^{1-\frac{N}{p}}
$$

desigualdade válida para $p \geq N$ se $N>2$ e para $p>N$ se $N=2$. Observe-se que a constante $C=C(N, p)$ não depende do tamanho do domínio. Além disso, usamos a desigualdade de Young na forma

$$
a b \leq \varepsilon a^{1 / \alpha}+\left(\frac{\alpha}{\varepsilon}\right)^{\frac{\alpha}{1-\alpha}}(1-\alpha) b^{\frac{1}{1-\alpha}},
$$

$\operatorname{com} \alpha=\frac{N}{2 p}$. 
Levando em conta (2.56), (2.57), da equação (2.52) obtém-se

$$
\begin{aligned}
& \frac{1}{2} \frac{\mathrm{d}}{\mathrm{d} t} \int_{\Omega} \rho_{1} U^{2} \mathrm{~d} x+\left(\mu+\mu_{r}-2 \varepsilon\right) \int_{\Omega}|\nabla U|^{2} \\
\leq & 2 \mu_{r}\left(\frac{\delta}{2}\|\nabla W\|_{L^{2}(\Omega)^{9}}^{2}+\frac{1}{2 \delta}\|U\|_{L^{2}(\Omega)^{3}}^{2}\right)+C_{3} \varepsilon^{-\frac{N}{2 p-N}}\|v\|_{L^{p}(\Omega)}^{\frac{2 p}{2 p-N}}\left(\|\eta\|_{L^{2}(\Omega)}^{2}+\|U\|_{L^{2}(\Omega)^{3}}^{2}\right) \\
& +\left\|\nabla u_{2}\right\|_{L^{\infty}(\Omega)^{3}}\|U\|_{L^{2}(\Omega)^{3}}^{2} .
\end{aligned}
$$

As constantes $\varepsilon, \delta, \delta_{1}$ serão escolhidas posteriormente de maneira conveniente.

De forma análoga ao que fizemos anteriormente, tomando o produto interno na. equação (2.53) com $W$ e integrando sobre $\Omega$, temos

$$
\begin{aligned}
& \int_{\Omega} \rho_{1}\left\{\frac{\partial W}{\partial t}+\left(u_{1} \cdot \nabla\right) W\right\} \mathrm{d} x=\frac{1}{2} \frac{\mathrm{d}}{\mathrm{d} t} \int_{\Omega} \rho_{1} W^{2} \mathrm{~d} x \\
& \int_{\Omega} A W \cdot W \mathrm{~d} x=\nu \int_{\Omega}|\nabla W|^{2} \mathrm{~d} x+\gamma \int_{\Omega}|\operatorname{div} W|^{2} \mathrm{~d} x \\
& \left|\int_{\Omega} \eta\left\{\frac{\partial w_{2}}{\partial t}+\left(u_{2} \cdot \nabla\right) w_{2}-g\right\} W \mathrm{~d} x\right|=\left|\int_{\Omega} \eta \bar{v} W \mathrm{~d} x\right| \\
\leq & \varepsilon\|\nabla W\|_{L^{2}(\Omega)^{3}}^{2}+C_{3} \varepsilon^{-\frac{N}{2 p-N}}\|\bar{v}\|_{L^{p}(\Omega)^{3}}^{\frac{2 p}{2 p-N}}\left(\|\eta\|_{L^{2}(\Omega)}^{2}+\|W\|_{L^{2}(\Omega)^{3}}^{2}\right) .
\end{aligned}
$$

Nesta última desigualdade, utilizamos o argumento feito anteriormente com $\bar{v}=$ $\frac{\partial w_{2}}{\partial t}+\left(u_{2} \cdot \nabla\right) w_{2}-g \in L^{p}\left(Q_{T_{1}}\right)$. Portanto, das expressões acima, junto com a equação (2.53), obtém-se a desigualdade

$$
\begin{aligned}
& \frac{1}{2} \frac{\mathrm{d}}{\mathrm{d} t} \int_{\Omega} \rho_{1} W^{2} \mathrm{~d} x+(\nu-2 \varepsilon) \int_{\Omega}|\nabla W|^{2} \mathrm{~d} x+(\gamma-\varepsilon) \int_{\Omega}|\operatorname{div} W|^{2} \mathrm{~d} x \\
\leq & 2 \mu_{\gamma}\left(\frac{\delta_{1}}{2}\|\nabla U\|_{L^{2}(\Omega)^{9}}^{2}+\frac{1}{2 \delta_{1}}\|W\|_{L^{2}(\Omega)^{3}}^{2}\right) \\
& +C_{3} \varepsilon^{-\frac{N}{2 p-N}}\|\bar{v}\|_{L^{p}(\Omega)}^{\frac{2 p}{2 p-N}}\left(\|\eta\|_{L^{2}(\Omega)}^{2}+\|W\|_{L^{2}(\Omega)^{3}}^{2}\right) \\
& +\left\|\nabla u_{2}\right\|_{L^{\infty}(\Omega)^{9}}\|U\|_{L^{2}(\Omega)^{3}}^{2}
\end{aligned}
$$

Similarmente, multiplicamos a equação (2.54) por $\eta$ e integrando sobre $\Omega$, vem que

$$
\frac{1}{2} \frac{\mathrm{d}}{\mathrm{d} t} \int_{\Omega} \eta^{2} \mathrm{~d} x+\int_{\Omega} \nabla \cdot\left(u_{1} \eta+U \rho_{2}\right) \eta=0
$$

Levando em conta que

$$
\int_{\Omega} \operatorname{div}\left(u_{1} \eta\right) \eta=\frac{1}{2} \int_{\Omega} \eta^{2} \operatorname{div}\left(u_{1}\right) \mathrm{d} x=0
$$


e que o outro termo satisfaz a desigualdade

$$
\begin{aligned}
& \left|\int_{\Omega} \operatorname{div}\left(U \rho_{2}\right) \eta \mathrm{d} x\right| \leq\left|\int_{\Omega} \nabla \rho_{2} \cdot U \eta \mathrm{d} x\right| \\
\leq & \varepsilon\|\nabla U\|_{L^{2}(\Omega)^{3}}+C_{3} \varepsilon^{-\frac{N}{2 p-N}}\left\|\nabla \rho_{2}\right\|_{L^{p}(\Omega)^{3}}^{\frac{2 p}{2 p-N}}\left(\|\eta\|_{L^{2}(\Omega)}^{2}+\|U\|_{L^{2}(\Omega)^{3}}^{2}\right) .
\end{aligned}
$$

Nesta última desigualdade utilizamos o argumento anterior com $v=\nabla \rho_{2}$. Assim, temos

$$
\frac{1}{2} \frac{\mathrm{d}}{\mathrm{d} t} \int_{\Omega} \eta^{2} \mathrm{~d} x \leq \varepsilon\|\nabla U\|_{L^{2}(\Omega)^{3}}^{2}+C_{3} \varepsilon^{-\frac{N}{2 p-N}}\left\|\nabla \rho_{2}\right\|_{L^{p}(\Omega)^{3}}^{\frac{2 p}{2 p-N}}\left(\|\eta\|_{L^{2}(\Omega)}^{2}+\|U\|_{L^{2}(\Omega)^{3}}^{2}\right)
$$

Escolhendo $\delta=\delta_{1}=\frac{\varepsilon}{\mu_{r}}$ e somando as desigualdades (2.58), (2.59) e (2.60), obtém-se

$$
\begin{aligned}
& \frac{1}{2} \frac{\mathrm{d}}{\mathrm{d} t}\left\{\int_{\Omega}\left(\rho_{1} U^{2}+\rho_{1} W^{2}+\eta^{2}\right) \mathrm{d} x\right\}+\left(\mu+\mu_{r}-4 \varepsilon\right) \int_{\Omega}|\nabla U|^{2} \mathrm{~d} x \\
& +(\nu-3 \varepsilon) \int_{\Omega}|\nabla W|^{2} \mathrm{~d} x+(\gamma-\varepsilon) \int_{\Omega}|\operatorname{div} W|^{2} \mathrm{~d} x+4 \mu_{r}\|W\|_{L^{2}(\Omega)^{3}}^{2} \\
\leq & {\left[\frac{\mu_{r}}{2 \varepsilon}+\left\|\nabla u_{2}\right\|_{L_{\infty}(\Omega)^{9}}^{2}+\left\|\nabla w_{2}\right\|_{L^{\infty}(\Omega)}^{2}+C_{3} \varepsilon^{-\frac{2 N}{2 p-N}}\left(\|v\|_{L^{p}(\Omega)^{3}}^{\frac{2 p}{2 p-N}}\right.\right.} \\
& \left.\left.+\|\bar{v}\|_{L^{p}(\Omega)^{3}}^{\frac{2 p}{2 p-N}}+\left\|\nabla \rho_{2}\right\|_{L^{p}(\Omega)^{3}}^{\frac{2 N}{2 p-N}}\right)\right]\left(\|U\|_{L^{2}(\Omega)^{3}}^{2}+\|W\|_{L^{2}(\Omega)^{3}}^{2}+\|\eta\|_{L^{2}(\Omega)}^{2}\right)
\end{aligned}
$$

O parâmetro $\varepsilon$ pode ser escolhido como $\varepsilon<\min \left\{\frac{\mu+\mu_{r}}{4}, \frac{\mu_{c}}{2}, \gamma\right\}$. A função que está dentro dos colchetes é uma função integrável e, portanto, se definirmos a função

$$
J(t)=\int_{\Omega}\left(\rho_{1} U^{2}+\rho_{1} W^{2}+\eta^{2}\right) \mathrm{d} x
$$

temos que $J(0)=0$ e pela desigualdade integral (2.61) vem que $J(t)=0$ para todo t. Logo, finalizamos a prova do teorema principal. 


\section{Capítulo 3}

\section{Soluções periódicas dos fluidos incompressíveis assimétricos não-homogêneos}

\subsection{Introdução}

Estudamos neste capítulo a existência de soluções fracas com a propriedade reprodutiva do sistema $(0.1)$ - (0.7) sobre um domínio $\Omega$ limitado em $\mathbb{R}^{3}$ com contorno $\partial \Omega$ de classe $C^{2}$, num intervalo de tempo $[0, T], 0<T<+\infty$.

Em primeiro lugar especificamos a noção de "reprodução". Seja $X$ um espaço de Banach onde consideramos a equação de evolução não-linear

$$
\left\{\begin{array}{l}
\frac{\mathrm{d} \phi}{\mathrm{d} t}=A(t, \phi), \\
\phi(0)=a_{0} .
\end{array}\right.
$$

Dizemos que (3.1) é reprodutiva ou que tem a propriedade de reprodução em $t_{0}$ se podemos encontrar $a_{0} \in X$ tal que $\phi\left(t_{0}\right)=a_{0}$. Dizemos que $\phi(t)$ é uma solução reprodutiva de (3.1) e $a_{0}$ é um valor inicial reprodutivo em $t_{0}$. Dizemos que (3.1) é reprodutiva se (3.1) é reprodutiva em todo $t_{0}$.

As principais notações foram apresentadas no Capítulo 1 e são, salvo mudanças óbvias, suficientes para o desenvolvimento deste capítulo. Em um interessante artigo, Kaniel e Shinbrot [33] provam a propriedade de reprodução das soluções fracas para 
equações de Navier-Stokes, quer dizer, soluções que satisfazem $u(0)=u(T)$. Esta noção generaliza o conceito de solução periódica, isto é, soluções que satisfazem $u(t+\omega)=u(t), \forall t \in \mathbb{R}$, sendo $\omega$ o período; toda solução periódica é reprodutiva, a recíproca, no entanto, não se verifica necessariamente. Porém, se tivéssemos a unicidade de soluções e se o campo externo for regular e $T$-periódico no tempo, a propriedade de reprodução de uma solução fornece uma solução fraca $T$-periódica. De fato, ela é uma solução forte e muito regular. Isto é devido a que podemos provar que $u(t) \in C^{\infty}(\Omega)$ para $t>0$ e qualquer dado inicial $u_{0} \in H$. Assim, $u_{p}(t) \in C^{\infty}(\Omega)$ para $t \in[T, 2 T]$ e pela $T$-periodicidade concluímos que $u_{p}(t)=u_{p}(t+T) \in C^{\infty}(\Omega)$ para $t \in[0, T]$. Em particular, devemos ter que $u_{p}(0) \in C^{\infty}(\Omega)$.

A prova original apresentada em [34] é difícil, ela foi simplificada por Lions [43]. Para o caso bidimensional, veja [84]. Para outras equações da mecânica dos fluidos podem ser vistos os trabalhos [17], [65], [76] e [87].

Neste capítulo, vamos estender estas idéias para o caso de fluidos não-homogêneos assimétricos. Basicamente usaremos uma combinação dos argumentos desenvolvidos em [65] para os fluidos magneto-micropolares com densidade constante e em [70], no qual é estudado o caso dos fluidos não-homogêneos sem velocidade micro-rotacional.

Depois destes breves comentários passamos a descrever a estrutura deste capítulo.

Na primeira seção, apresentamos o problema que será abordado, definiremos a noção de solução reprodutiva, assim como a noção de solução fraca que será usada e enunciamos o resultado.

Na Seção 2, tratamos da existência de soluções fracas periódicas de um problema linear auxiliar com regularização elíptica (Teorema 3.2).

Finalmente, na Seção 3, obtemos estimativas convenientes para as funções $u^{(k)} \mathrm{e}$ $w^{(k)}$ que nos permitem passar ao limite nas equações e concluir a prova do Teorema 1, ou seja, do resultado principal. Para não sobrecarregar a notação, a norma $L_{2}(\Omega)$ 
de uma função será, às vezes, denotada simplesmente por $\|$ · $\|$.

\subsection{Definição e apresentação do problema}

Vamos assumir que sobre a fronteira $\partial \Omega$ de $\Omega$ são satisfeitas as seguintes condições:

$$
u(x, t)=w(x, t)=0, \quad(x, t) \in \partial \Omega \times[0, T]
$$

e definimos solução reprodutiva fraca do problema abaixo.

Definição 3.1 Sejam $f, g \in L^{2}\left(0, T, L^{2}(\Omega)^{3}\right)$ periódicas de periodo $T<+\infty, \rho_{0}(x) \in$ $L^{\infty}(\Omega), 0<m \leq \rho_{0} \leq M$. Então $(\rho, u, w)$ é uma solução reprodutiva fraca do problema (0.1) - (0.7) em $Q_{T}$ se

(i) $u \in L^{\infty}\left(0, T ; L^{2}(\Omega)\right) \cap L^{2}(0, T ; V)$;

$w \in L^{\infty}\left(0, T ; L^{2}(\Omega)\right) \cap L^{2}\left(0, T ; H^{1}(\Omega) ;\right.$

$\rho \in L^{\infty}\left(Q_{T}\right) ; u(0)=u(T) ; w(0)=w(T) ; \rho(0)=\rho(T) ;$

(ii) $\forall \varphi \in \Phi, \psi \in \Psi$, tem-se as desigualdades integrais

$$
\begin{aligned}
& -\int_{0}^{T}\left\{\left(\rho u, \frac{\partial \varphi}{\partial t}\right)+(\rho u,(u \cdot \nabla) \varphi)+\left(\mu+\mu_{r}\right)(\nabla u, \nabla \varphi)\right. \\
& \left.\quad-2 \mu_{r}(\operatorname{rot} w, \varphi)-(\rho f, \varphi)\right\} \mathrm{d} t \\
& =\left(\rho_{0} u_{0}, \varphi(0)\right)-(\rho(T) u(T), \varphi(T))
\end{aligned}
$$

e para a equação do momentum angular

$$
\begin{aligned}
& -\int_{0}^{T}\left\{\left(\rho w, \frac{\partial \psi}{\partial t}\right)+(\rho u,(w \cdot \nabla) \psi)+\nu(\nabla w, \nabla \psi)\right. \\
& \left.+\gamma(\operatorname{div} w, \operatorname{div} \psi)+4 \mu_{r}(w, \psi)-2 \mu_{r}(\operatorname{rot} u, \psi)-(\rho g, \psi)\right\} \mathrm{d} t \\
& =\left(\rho_{0} w_{0}, \psi(0)\right)-(\rho(T) w(T), \psi(T))
\end{aligned}
$$

onde 


$$
\begin{aligned}
& \Phi=\left\{\varphi \mid \varphi \in L^{2}(0, T ; V) ; \frac{\partial \varphi}{\partial t} \in L^{2}\left(0, T ; L^{6 / 5}(\Omega)\right), \frac{\partial \varphi}{\partial x_{j}} \in L^{\infty}\left(0, T ; L^{3 / 2}(\Omega)\right), j=1,2,3\right\} \\
& \Psi=\left\{\psi \mid \psi \in L^{2}\left(0, T ; L^{2}(\Omega)\right) ; \frac{\partial \psi}{\partial t} \in L^{2}\left(0, T ; L^{2}(\Omega)\right) ; \frac{\partial \psi}{\partial x_{j}} \in L^{\infty}\left(0, T ; L^{3 / 2}(\Omega), j=1,2,3\right\}\right.
\end{aligned}
$$

Se multiplicarmos (0.4) por $u$ e somando o resultado na primeira equação $(0.1)$, obtém-se

$$
\frac{\partial}{\partial t}(\rho u)+\rho(u \cdot \nabla) u+u(u-\nabla) \rho-\left(\mu+\mu_{r}\right) \Delta u+\nabla p=2 \mu_{r} \operatorname{rot} w+\rho f .
$$

Usando o fato de que $\operatorname{div} u=0$ e que

$$
\nabla \cdot(u \rho u)=\sum_{j=1}^{3} \frac{\partial}{\partial x_{j}}\left(u_{j} \rho u\right)=\rho(u \cdot \nabla) u+u(u \cdot \nabla) \rho
$$

obtém-se

$$
\frac{\partial}{\partial t}(\rho u)+\nabla \cdot(u \rho u)-\left(\mu+\mu_{r}\right) \Delta u=2 \mu_{r} \operatorname{rot} w+\rho f
$$

Usando o mesmo argumento para a equação (0.3), mas agora multiplicamos (0.4) por $w$ e o resultado é somado a (0.3) e levando em conta que

$$
\nabla(u \rho w)=(u \cdot \nabla) \rho w+\rho(u \cdot \nabla) w
$$

obtém-se

$$
\frac{\partial}{\partial t}(\rho w)+\nabla \cdot(u \rho w)-\nu \Delta w-\gamma \nabla \operatorname{div} w+4 \mu_{r} w=2 \mu_{r} \operatorname{rot} u+\rho g
$$

Multiplicando (3.2) por $\varphi \in \Phi$ e (3.3 por $\psi$ e $\Psi$ e integrando por partes obtém-se o resultado desejado.

Os espaços $\Phi$ e $\Psi$ são escolhidos de forma que as integrações façam sentido. Como por exemplo, dado que pela imersão $V \subset\left(L^{6}(\Omega)\right)^{3}$ os termos da forma $u_{j} \rho u_{i} \in$ $L^{1}\left(0, T ; L^{3}(\Omega)\right)$ e, portanto, a integral $\int_{0}^{T}(\rho u,(u \cdot \nabla) \varphi) \mathrm{d} t$ faz sentido desde que $\frac{\partial \varphi}{\partial x_{j}} \in$ $L^{\infty}\left(0, T ; L^{3 / 2}(\Omega)\right)$. 
Observemos ainda que $\{u, w, \rho\}$ é solução fraca do sistema de equações (0.1) (0.7), as condições iniciais e de fronteira fazem sentido. De fato, se $u \in L^{2}(0, T ; V)$, $w \in L^{2}\left(0, T ; H_{0}^{1}\right), \rho \in L^{\infty}\left(Q_{T}\right)$, observe-se que

$$
\langle\operatorname{div}(\rho u), \varphi\rangle_{H^{-1} V}=-\langle\rho u, \nabla \varphi\rangle
$$

implicando

$$
\sup _{\|\varphi\|_{V} \leq 1}|\langle\operatorname{div}(\rho u), \varphi\rangle| \leq\|\rho\|_{L^{\infty}}\|u\|_{V}
$$

Portanto,

$$
\operatorname{div}(\rho u) \in L^{2}\left(0, T ; H^{-1}(\Omega)^{3}\right) .
$$

Pelo Lema 1.4 e da equação (0.4). Tomando $B=H^{-1}(\Omega)$ e como a imersão de $L^{\infty}(\Omega) \hookrightarrow H^{-1}(\Omega)$ é compacta, obtém-se que $\rho \in C\left([0, T] ; H^{-1}\right)$ e portanto $\rho(x, 0)=$ $\rho_{0}(x)$ faz sentido.

Nosso objetivo principal é provar o seguinte resultado, para o problema $(0.1)$ (0.7).

Teorema 3.2 Sejam $f, g \in L^{2}\left(0, T ; L^{2}(\Omega)^{3}\right)$ funções periódicas de periodo $T<$ $+\infty, \rho_{0} \in L^{\infty}(\Omega), 0<m \leq \rho_{0}<M$. Então existe uma solução fraca que possui a propriedade reprodutiva.

\subsection{Problema auxiliar e regularização elíptica}

Para demonstrar o Teorema 3.2, vamos usar o problema auxiliar (3.4) - (3.6) abaixo, que consiste em um problema linear com uma regularização elíptica.

Para isto consideraremos uma família de aproximações internas $V_{k} \subset V$ e $H_{k} \subset$ $H_{0}^{1}(\Omega)^{3}$. Assume-se que $V_{k}$ e $H_{k}$ são subespaços de dimensão $k$ e que para $v \in V$, $w \in H$, existem seqüencias $\left\{v_{k}\right\}$ e $\left\{w_{k}\right\}$ tais que $v_{k} \mapsto v, w_{k} \mapsto w$ quando $k \mapsto$ $+\infty$. Além disso, todas as componentes de $v$ em $V_{k}$ e $w$ em $H_{k}$ são suficientemente regulares. Denotamos por $P_{k}$ o operador de projeção de $V$ em $V_{k}$. 
Consideremos o problema auxiliar como o seguinte sistema:

$$
\begin{aligned}
& \rho^{k} \frac{\partial u^{k}}{\partial t}+\left(\rho^{k} P_{k} u^{(k-1)} \cdot \nabla\right) u^{k}-\left(\mu+\mu_{r}\right) \Delta u^{k}=2 \mu_{r} \operatorname{rot} w^{k}+\rho^{k} f-\frac{1}{2 k} \Delta \rho^{k}(3) \\
& \begin{aligned}
\rho^{k} \frac{\partial w^{k}}{\partial t} & +\left(\rho^{k} P_{k} u^{(k-1)} \cdot \nabla\right) w^{(k-1)}-\nu \Delta w^{k}-\gamma \nabla \operatorname{div} w^{k}+4 \mu_{r} w^{k} \\
\quad & =2 \mu_{r} \operatorname{rot} u^{k}+\rho^{k} g-\frac{1}{2 k} \Delta \rho^{k} w^{k}
\end{aligned} \\
& \frac{\partial \rho^{k}}{\partial t}+\left(P_{k} u^{(k-1)} \cdot \nabla\right) \rho^{k}=\frac{1}{k} \Delta \rho^{k}
\end{aligned}
$$

onde $k \geq 1$ inteiro, $u^{0} \in V, w^{0} \in H_{0}^{1}(\Omega)^{3}$, satisfazendo

$$
\begin{aligned}
& u^{k}(0, x)=u_{0}^{k}, \quad w^{k}(0, x)=w_{0}^{k}, \\
& u^{k}(t, x)=w^{k}(t, x)=0, \quad(t, x) \in[0, T] \times \partial \Omega, \\
& \rho^{k}(0, x)=\rho_{0}^{k}(x), \quad \frac{\partial \rho^{k}}{\partial \nu}=0, \quad(t, x) \in[0, T] \times \partial \Omega .
\end{aligned}
$$

Definamos o espaço

$$
W=\left\{\left.\varphi \in H^{2}(\Omega) \frac{\partial \varphi}{\partial \mathbf{n}}\right|_{\Gamma}=0\right\} .
$$

A seguir, definiremos a solução fraca do sistema de equações auxiliares (3.4) (3.9).

Definição 3.3 As funções $u^{k}, w^{k}$, $\rho^{k}$ são ditas soluções fracas do sistema (3.4) (3.9) $\mathrm{se}$

(i) $u^{k} \in L^{\infty}(0, T ; H) \cap L^{2}(0, T ; V)$,

$$
\begin{aligned}
& w^{k} \in L^{\infty}\left(0, T ; L^{2}(\Omega)^{3}\right) \cap L^{2}\left(0, T, H_{0}^{1}(\Omega)^{3}\right), \\
& \rho^{k} \in L^{\infty}\left(0, T ; H^{1}(\Omega)\right) \cap L^{2}(0, T ; W) \cap H^{1}\left(0, T ; L^{2}(\Omega)\right) \cap L^{\infty}\left(Q_{T}\right) ;
\end{aligned}
$$

(ii) A equação (3.6) é satisfeita qtp em $Q_{T}$;

(iii) Satisfazem as seguintes identidades integrais para todo $\varphi \in L^{2}(0, T, V)$ e $\psi \in$ $L^{2}\left(0, T ; H_{0}^{1}(\Omega)^{3}\right):$

$$
\int_{0}^{T}\left\{\left(\rho^{k} u^{k}\right)_{t}, \varphi\right)+\left(\rho^{k} P_{k} u^{(k-1)},\left(u^{k} \cdot \nabla\right) \varphi\right)+\left(\nabla u^{k}, \nabla \varphi\right)-\left(2 \mu_{r} \operatorname{rot} w^{k}, \varphi\right)
$$




$$
\begin{aligned}
& \left.+\frac{1}{2 k}\left(\Delta \rho^{k} u^{k}, \varphi\right)-\left(\rho^{k} f, \varphi\right)\right\} \mathrm{d} t=0 \\
& \int_{0}^{T}\left\{\left(\left(\rho^{k} w^{k}\right)_{t}, \psi\right)+\left(\rho^{k} P_{k} u^{(k-1)},\left(w^{k} \cdot \nabla\right) \psi\right)+B\left(w^{k}, \psi\right)+4 \mu_{r}\left(w^{k}, \psi\right)\right. \\
& \left.-2 \mu_{r}\left(\operatorname{rot} u^{k}, \psi\right)+\frac{1}{2 k}\left(\Delta \rho^{k} w^{k}, \psi\right)-\rho^{k} g\right\} \mathrm{d} t=0,
\end{aligned}
$$

onde

$$
B(w, \psi)=\nu(\nabla w, \nabla \psi)+\gamma(\operatorname{div} w, \operatorname{div} \psi)
$$

Para mostrar que o sistema (3.4) - (3.9) possui solução única, usamos o método de Galerkin, que consiste em considerar conjuntos completos $\left\{\varphi_{i}(x)\right\}_{i=1}^{\infty}$ e $\left\{\phi_{i}(x)\right\}_{i=1}^{\infty}$ dos espaços $V$ e $H_{0}^{1}(\Omega)$, respectivamente, e as aproximações de Galerkin

$$
u_{n}^{k}(x, t)=\sum_{i=1}^{n} c_{i n}(t) \varphi_{i}(x), \quad w_{n}^{k}(x, t)=\sum_{i=1}^{n} d_{i n}(t) \phi_{i}(x)
$$

Para $u_{n}^{k}$ e $w_{n}^{(k)}$, respectivamente, satisfazendo as equações seguintes

$$
\begin{aligned}
& \left(\rho^{k} \frac{\partial u_{n}^{k}}{\partial t}, \varphi_{j}\right)+\left(\left(\rho^{k} P_{k} u^{(k-1)} \cdot \nabla\right) u_{n}^{k}, \varphi_{j}\right)+\left(\nabla u_{n}^{k}, \nabla \varphi_{j}\right) \\
& =\left(\rho^{k} f, \varphi_{j}\right)+2 \mu_{r}\left(\operatorname{rot} w_{n}^{k}, \varphi_{j}\right)-\frac{1}{2 k}\left(\Delta \rho^{k} u_{n}^{k}, \varphi_{j}\right) \\
& \left(\rho^{k} \frac{\partial w^{k}}{\partial t}, \phi_{j}\right)+\left(\left(\rho^{k} P_{k} u^{(k-1)} \cdot \nabla\right) w_{n}^{k}, \phi_{j}\right)+\left(\nabla w_{n}^{k}, \nabla \phi_{j}\right)+4 \mu_{r}\left(w_{k}^{k}, \phi_{j}\right) \\
& =2 \mu_{r}\left(\operatorname{rot} u_{n}^{k}, \phi_{j}\right)+\left(\rho^{k} g, \phi_{j}\right)-\frac{1}{2 k}\left(\Delta \rho^{k} w_{n}^{k}, \phi_{j}\right)
\end{aligned}
$$

com as condições iniciais

$$
u_{n}^{k}(x, 0)=u_{0 n}^{k}, \quad w_{n}^{k}(x, 0)=w_{0 n}^{k} .
$$

O sistema (3.10) - (3.11) junto com as condições iniciais é um sistema de equações diferenciais ordinárias e de uma forma padrão se prova que existe uma única solução $\left(u_{n}^{k}, w_{n}^{k}\right)$ num intervalo $\left[0, T_{n}\right), \forall n \in \mathbb{N}$. Usando as estimativas a priori para o $\left(u_{n}^{k}, w_{n}^{k}\right)$, obtidas no Teorema 3.4 abaixo, podemos obter um intervalo $[0, T)$ onde as soluções podem ser definidas para todo $n \in \mathbb{N}$.

Agora faremos algumas estimativas a priori que posteriormente serão úteis. 


\subsubsection{Estimativas a priori para a densidade}

Primeiro observemos que pelo princípio do máximo obtém-se

$$
0<m \leq \rho^{k}(x, t) \leq M
$$

Multiplicando (3.6) por $\rho^{k}$ e fazendo integração por partes, obtemos

$$
\begin{aligned}
\frac{1}{2} \frac{\mathrm{d}}{\mathrm{d} t}\left\|\rho^{k}\right\|^{2}+\frac{1}{k}\left\|\nabla \rho^{k}\right\|^{2} & =-\int_{\Omega}\left(P_{k} u^{(k-1)} \cdot \nabla\right) \rho^{k} \rho^{k} \mathrm{~d} k \\
& =-\int_{\Omega}\left(P_{k} u^{(k-1)} \cdot \nabla\right) \frac{\left|\rho^{k}\right|^{2}}{2} \mathrm{~d} k \\
& =-\int_{\Omega} \operatorname{div}\left(P_{k} u^{(k-1)}\right) \frac{\left|\rho^{k}\right|^{2}}{2} \mathrm{~d} k
\end{aligned}
$$

Portanto,

$$
\frac{1}{2} \frac{\mathrm{d}}{\mathrm{d} t}\left\|\rho^{k}\right\|^{2}+\frac{1}{k}\left\|\nabla \rho^{k}\right\|^{2}=0
$$

e, conseqüentemente,

$$
\frac{1}{2}\left\|\rho^{k}\right\|^{2}+\frac{1}{k} \int_{0}^{t}\left\|\nabla \rho^{k}(s)\right\|^{2} \mathrm{~d} s=\frac{1}{2}\left\|\rho^{k}(0)\right\|^{2}=\frac{1}{2}\left\|\rho_{0}\right\|^{2} .
$$

Portanto,

$$
\frac{1}{k} \int_{0}^{t}\left\|\nabla \rho^{k}(s)\right\|^{2} \mathrm{~d} s \leq C, \quad \sup _{t}\left\|\rho^{k}(t)\right\| \leq C .
$$

Como $T$ é finito, temos as estimativas

$$
\begin{aligned}
\left\|\rho^{k}\right\|_{L^{\infty}\left(0, T ; L^{2}(\Omega)\right)} & \leq C, \\
\frac{1}{k}\left\|\rho^{k}\right\|_{L^{\infty}\left(0, T ; H^{1}(\Omega)\right)} & \leq C .
\end{aligned}
$$

Multiplicando a equação (3.6) por $-\Delta \rho^{k}$ e integrando sobre $\Omega$, obtém-se

$$
\begin{aligned}
\frac{1}{2} \frac{\mathrm{d}}{\mathrm{d} t}\left\|\nabla \rho^{k}\right\|^{2}+\frac{1}{k}\left\|\Delta \rho^{k}\right\| & =\left(\left(\rho_{k} u^{(k-1)} \cdot \nabla\right) \rho^{k}, \Delta \rho^{k}\right) \\
& =-\left(\left(\nabla \rho^{k} \cdot \nabla\right) P_{k} u^{(k-1)}, \nabla \rho^{k}\right) \\
& \leq\left\|\nabla P_{k} u^{(k-1)}\right\|\left\|\nabla \rho^{k}\right\|_{L^{4}}^{2} \\
& \leq C\left\|u^{(k-1)}\right\|_{V}\left\|\nabla \rho^{k}\right\|_{L^{4}}^{2}
\end{aligned}
$$


Usando a desigualdade de interpolação, obtemos

$$
\left\|\nabla \rho^{k}\right\|_{L^{4}} \leq\left\|\Delta \rho^{k}\right\|\left\|\rho^{k}\right\|_{L^{\infty}} \leq C\left\|\Delta \rho^{k}\right\|
$$

e, conseqüentemente,

$$
\frac{\mathrm{d}}{\mathrm{d} t}\left\|\nabla \rho^{k}\right\|^{2}+\frac{1}{k}\left\|\Delta \rho^{k}\right\|^{2} \leq C k\left\|u^{(k-1)}\right\|_{V}^{2}
$$

Fazendo integração de 0 a $t$, temos finalmente

$$
\begin{aligned}
\left\|\nabla \rho^{k}(t)\right\|^{2}+\frac{1}{k} \int_{0}^{t}\left\|\Delta \rho^{k}(s)\right\|^{2} \mathrm{~d} s & \leq C k \int_{0}^{t}\left\|u^{(k-1)}(s)\right\|_{V}^{2}+\left\|\nabla \rho^{k}(0)\right\|^{2} \\
& \leq C k .
\end{aligned}
$$

Assim,

$$
\frac{1}{k} \int_{0}^{t}\left\|\Delta \rho^{k}(s)\right\|^{2} \mathrm{~d} s \leq C
$$

No próximo teorema estabelecemos um resultado de existência e unicidade para o sistema (3.4) - (3.9) de soluções reprodutivas fracas.

\subsection{Soluções reprodutivas do problema regulari- zado}

Teorema 3.4 Assumamos que $f \in L^{2}(0, T ; H), g \in L^{2}\left(0, T ; L^{2}(\Omega)^{3}\right)$ periódicas de período $T<\infty, u_{0}^{k} \in H, w_{0}^{k} \in L^{2}(\Omega), \rho_{0}^{k} \in H^{1}(\Omega), 0<m \leq \rho_{0}^{k} \leq M \leq 1$. Então existe uma única solução fraca do sistema (3.4) - (3.9) tal que

$$
\begin{aligned}
& u^{k} \in C([0, T] ; H), \quad w^{k} \in C\left([0, T] ; L^{2}(\Omega)^{3}\right), \\
& \left(\rho^{k} u^{k}\right)_{t} \in L^{1}\left(0, T ; V^{*}\right), \quad\left(\rho^{k} w\right)_{t} \in L^{1}\left(0, T ; H^{-1}(\Omega)^{3}\right), \\
& u_{0}^{k}=u^{k}(T), \quad w_{0}^{k}=w^{k}(T), \quad \rho_{0}^{k}=\rho^{k}(T) .
\end{aligned}
$$

Demonstração: A prova do teorema será feita em três etapas.

(i) Estimativa a priori básicas para $u_{n}^{k}$ e $w_{n}^{k}$ 
Multiplicando a equação (3.4) por $2 u_{n}^{k}$ e integrando sobre $\Omega$, obtemos

$$
\begin{aligned}
& 2\left(\rho\left(u_{n}^{k}\right) t, u_{n}^{k}\right)+\left(\mu+\mu_{r}\right)\left(\nabla u_{n}^{k}, \nabla u_{n}^{k}\right)=4 \mu_{r}\left(\operatorname{rot} w_{n}^{k}, u_{n}^{k}\right)-\frac{1}{k}\left(\Delta \rho^{k} u_{n}^{k}, u_{n}^{k}\right) \\
& -2\left(\left(\rho^{k} P_{k} u^{(k-1)} \cdot \nabla\right) u_{n}^{k}, u_{n}^{k}\right)
\end{aligned}
$$

Além disso, observemos que

$$
2\left(\rho^{k}\left(u_{n}^{k}\right)_{t}, u_{n}^{k}\right)=\frac{\mathrm{d}}{\mathrm{d} t}\left\|\sqrt{\rho^{k}} u_{n}^{k}\right\|^{2}-\left(\rho_{k}^{t} u_{n}^{k}, u_{n}^{k}\right)
$$

e da equação (3.6), obtém-se

$$
\left(\rho_{t}^{k} u_{n}^{k}, u_{n}^{k}\right)=-\left(\left(\rho_{k} u^{k-1} \cdot \nabla\right) \rho^{k}, u_{n}^{k}\right)+\frac{1}{k}\left(\Delta \rho^{k}, u_{n}^{k}\right)
$$

Substituindo estas últimas expressões em (3.15),

$$
\begin{aligned}
& 2\left(\rho^{k}\left(u_{n}^{k}\right)_{t}, u_{n}^{k}\right)-2\left(\left(\rho^{k} P_{k} u^{k-1} \cdot \nabla\right) u_{n}^{k}, u_{n}^{k}\right)-\frac{1}{2 k}\left(\Delta \rho^{k} u_{n}^{k}, u_{n}^{k}\right) \\
= & -\left(\left(\rho_{k} u^{k-1} \cdot \nabla\right) \rho^{k}, u_{n}^{k}\right)-2\left(\left(\rho^{k} P_{k} u^{k-1} \cdot \nabla\right) u_{n}^{k}, u_{n}^{k}\right) .
\end{aligned}
$$

Como $\operatorname{div}\left(P_{k} u^{k-1}\right)=0$, temos

$$
\left(P_{k} u^{k-1} \cdot \nabla\right) \rho^{k}=\operatorname{div}\left(\rho^{k} P_{k} u^{k-1}\right)
$$

e, portanto,

$$
-\left(\left(P_{k} u^{k-1} \cdot \nabla\right) \rho^{k}, u_{n}^{k}\right)=-\left(\operatorname{div}\left(\rho^{k} P_{k} u^{k-1}\right) u_{n}^{k}, u_{n}^{k}\right)=2\left(\left(\rho^{k} P_{k} u^{k-1} \cdot \nabla\right) u_{n}^{k}, u_{n}^{k}\right) .
$$

Logo,

$$
2\left(\rho^{k}\left(u_{n}^{k}\right)_{t}, u_{n}^{k}\right)-2\left(\left(\rho^{k} P_{k} u^{k-1} \cdot \nabla\right) u_{n}^{k}, u_{n}^{k}\right)-\frac{1}{2 k}\left(\Delta \rho^{k} u_{n}^{k}, u_{n}^{k}\right)=0
$$

Usando estes resultados, obtém-se

$$
\frac{\mathrm{d}}{\mathrm{d} t}\left\|\sqrt{\rho^{k}} u_{n}^{k}\right\|^{2}+2\left(\mu+\mu_{r}\right)\left\|\nabla u_{n}^{k}\right\|^{2}=4 \mu_{r}\left(\operatorname{rot} w_{n}^{k}, u_{n}^{k}\right)+2\left(\rho^{k} f, u_{n}^{k}\right) .
$$

Por outro lado, multiplicando (1.3) por $2 w_{n}^{k}$ e integrando sobre $\Omega$, obtém-se

$$
\begin{aligned}
& 2\left(\rho^{k}\left(w_{n}^{k}\right)_{t}, w_{n}^{k}\right)+2\left(\left(\rho^{k} P_{k} u^{k-1} \cdot \nabla\right) w_{n}^{k}, w_{n}^{k}\right)+2 \nu\left\|\nabla w_{n}^{k}\right\|^{2} \\
& +2 \gamma\left\|\operatorname{div} w_{n}^{h}\right\|^{2}+8 \mu_{r}\left\|w_{n}^{k}\right\|^{2}=4 \mu_{r}\left(\operatorname{rot} u_{n}^{k}, w_{n}^{k}\right)+2\left(\rho^{k} g, w_{n}^{k}\right)-\left(\frac{1}{k} \Delta \rho^{k} w_{n}^{k}, w_{n}^{k}\right) .
\end{aligned}
$$


Analogamente ao feito anteriormente, temos

$$
2\left(\rho^{k}\left(w_{n}^{k}\right)_{t}, w_{n}^{k}\right)-2\left(\left(\rho^{k} P_{k} u^{k-1} \cdot \nabla\right) w_{n}^{k}, w_{n}^{k}\right)-\frac{1}{k}\left(\Delta \rho^{k} w_{n}^{k}, w_{n}^{h}\right)=0 .
$$

Assim, obtemos

$$
\begin{aligned}
& \frac{\mathrm{d}}{\mathrm{d} t}\left\|\sqrt{\rho^{k}} w_{n}^{k}\right\|^{2}+2 \nu\left\|\nabla w_{n}^{k}\right\|^{2}+2 \gamma\left\|\operatorname{div} w_{n}^{k}\right\|^{2}+8 \mu_{r}\left\|w_{n}^{k}\right\|^{2} \\
& =4 \mu_{r}\left(\operatorname{rot} u_{n}^{k}, w_{n}^{k}\right)+\left(\rho^{k} g, w_{n}^{k}\right) .
\end{aligned}
$$

Somando as igualdades (3.16) e (3.17), obtemos

$$
\begin{aligned}
& \frac{\mathrm{d}}{\mathrm{d} t}\left(\left\|\sqrt{\rho^{k}} u_{n}^{k}\right\|+\left\|\sqrt{\rho^{k}} w_{n}^{k}\right\|^{2}\right)+2\left(\mu+\mu_{r}\right)\left\|\nabla u_{n}^{k}\right\|^{2} \\
& +2 \nu\left\|\nabla w_{n}^{k}\right\|^{2}+2 \gamma\left\|\operatorname{div} w_{n}^{k}\right\|^{2}+8 \mu_{r}\left\|w_{n}^{k}\right\|^{2} \\
& =2\left(\rho^{k} f, u_{n}^{k}\right)+\left(\rho^{k} g, w_{n}^{k}\right)+8 \mu_{r}\left(\operatorname{rot} u_{n}^{k}, w_{n}^{k}\right) .
\end{aligned}
$$

A seguir faremos estimativas para o lado direito de (3.18). Assim,

$$
\begin{aligned}
2\left(\rho^{k} f, u_{n}^{k}\right) & \leq 2\left\|\rho^{k}\right\|_{L^{\infty}}\|f\|\left\|u_{n}^{k}\right\| \leq C_{\varepsilon}\|f\|^{2}+\varepsilon\left\|\nabla u_{n}^{k}\right\|^{2}, \\
2\left(\rho^{k} g, w_{n}^{k}\right) & \leq 2\left\|\rho^{k}\right\|\|g\|\left\|w_{n}^{k}\right\| \leq C_{8}\|g\|^{2}+\delta\left\|\nabla w_{n}^{k}\right\|^{2}, \\
8 \mu_{r}\left(\operatorname{rot} u_{n}^{k}, w_{n}^{k}\right) & \leq 8 \mu_{r}\left\|w_{n}^{k}\right\|\left\|\operatorname{rot} u_{n}^{k}\right\|=8 \mu_{r}\left\|w_{n}^{k}\right\|\left\|\nabla u_{n}^{k}\right\| \\
& =8 \mu_{r}\left\|w_{n}^{k}\right\|^{2}+2 \mu_{r}\left\|\nabla u_{n}^{k}\right\| .
\end{aligned}
$$

Usando estas estimativas em (3.18), obtemos a desigualdade

$$
\begin{aligned}
& \frac{\mathrm{d}}{\mathrm{d} t}\left(\left\|\sqrt{\rho^{k}} u_{n}^{k}\right\|^{2}+\left\|\sqrt{\rho^{k}} w_{n}^{k}\right\|^{2}\right)+2 \mu_{r}\left\|\nabla u_{n}^{k}\right\|^{2}+C_{1}\left\|\nabla w_{n}^{k}\right\|^{2}+C_{2}\left\|\operatorname{div} w_{n}^{k}\right\|^{2} \\
\leq & C\left(\|f\|^{2}+\|g\|^{2}\right) .
\end{aligned}
$$

Integrando a estimativa (3.19) de 0 até $t$, obtém-se

$$
\begin{aligned}
& u_{n}^{k} \in L^{2}(0, T ; V) \cap L^{\infty}(0, T ; H), \\
& w_{n}^{k} \in L^{2}\left(0, T ; H_{0}^{1}(\Omega)^{3}\right) \cap L^{\infty}\left(0, T ; L^{2}(\Omega)^{3}\right)
\end{aligned}
$$


uniformemente em $k$ e $n$.

Estas estimativas implicam quando $n \mapsto+\infty$

$$
\begin{aligned}
& u_{n}^{k} \rightarrow u^{k} \text { fraco em } L^{2}(0, T ; V), \\
& u_{n}^{k} \mapsto u^{k} \text { fraco }{ }^{*} \operatorname{em~} L^{\infty}(0, T ; H(\Omega))
\end{aligned}
$$

e

$$
\begin{aligned}
w_{n}^{k} & \mapsto w^{k} \text { fraco em } L^{2}\left(0, T ; H_{0}^{1}(\Omega)^{3}\right), \\
w_{n}^{k} & \mapsto w^{k} \text { fraco }^{*} \operatorname{em~} L^{\infty}\left(0, T ; L^{2}(\Omega)^{3}\right)
\end{aligned}
$$

Agora mostraremos que $\left(\rho^{k} u_{n}^{k}\right)_{t} \in L^{1}\left(0, T ; V^{k}\right)$ uniformemente em $n$. Multipliquemos a equação (3.4) por $u_{n}^{k}$ e somemos esta equação a (3.21). Obtemos

$$
\begin{aligned}
\frac{\partial}{\partial t}\left(\rho^{k} u_{n}^{k}\right)= & 2 \mu_{r} \operatorname{rot} w_{n}^{k}+\rho^{k} f+\left(\mu+\mu_{r}\right) \Delta u_{n}^{k}+\frac{1}{2 k} \Delta \rho^{k} u_{n}^{k} \\
& -\left(\rho^{k} P_{k} u^{k-1} \cdot \nabla\right) u_{n}^{k}-\left(P_{k} u_{n}^{k-1} \cdot \nabla\right) \rho^{k} u_{n}^{k} .
\end{aligned}
$$

Observe-se que

$$
\begin{aligned}
&\left\|2 \mu_{r} \operatorname{rot} w_{n}^{k}\right\|_{V^{*}}=2 \mu_{r} \sup _{\|\phi\|_{V} \leq 1}\left\langle\operatorname{rot} w_{n}^{k}, \phi\right\rangle=2 \mu_{r} \sup _{\|\phi\|_{V} \leq 1}\left(w_{n}^{k}, \phi\right) \\
& \leq 2 \mu_{r} \sup _{\|\phi\|_{V} \leq 1}\left\|w_{n}^{k}\right\|\|\operatorname{rot} \phi\|=2 \mu_{r} \sup _{\|\phi\|_{V} \leq 1}\left\|w_{n}^{k}\right\|\|\phi\|_{V} \\
&=2 \mu_{r}\left\|w_{n}^{k}\right\|, \\
&\left\|\rho^{k} f\right\|=\sup _{\|\phi\|_{V} \leq 1}\left\langle\rho^{k} f, \phi\right\rangle \leq\left\|\rho^{k}\right\|_{L^{\infty}}\|f\|_{1}, \\
&\left\|\left(\mu+\mu_{r}\right) \Delta u_{n}^{k}\right\|_{V^{*}}=\left(\mu+\mu_{r}\right) \sup _{\|\phi\|_{V} \leq 1}\left\langle\Delta u_{n}^{k}, \phi\right\rangle=\left(\mu+\mu_{r}\right) \sup _{\|\phi\|_{V} \leq 1}\left(\nabla u_{n}^{k}, \phi\right) \\
& \leq\left(\mu+\mu_{r}\right)\left\|\nabla u_{n}^{k}\right\|, \\
&\left\|\frac{1}{k} \Delta \rho^{k} u_{n}^{k}\right\|_{V^{*}} \frac{1}{k} \sup _{\|\phi\|_{V} \leq 1}\left\langle\Delta \rho^{k} u_{n}^{k}, \phi\right\rangle \leq \frac{1}{k} \sup _{\|\phi\|_{V} \leq 1}\left\|\Delta \rho^{k}\right\|\left\|u_{n}^{k}\right\|_{L^{4}}\|\phi\|_{L^{4}} \\
& \leq \frac{C}{k} \sup _{\|\phi\|_{V} \leq 1}\left\|\Delta \rho^{k}\right\|\left\|\nabla u_{n}^{k}\right\|\|\phi\|_{V} \\
& \leq \frac{C}{k}\left\|\Delta \rho^{k}\right\|\left\|\nabla u_{n}^{k}\right\|,
\end{aligned}
$$




$$
\begin{aligned}
& \left\|\left(\rho^{k} P_{k} u^{k-1} \cdot \nabla\right) u_{n}^{k}+\left(P_{k} u^{k-1} \cdot \nabla\right) \rho^{k} u_{n}^{k}\right\|_{V^{*}}=\sup _{\|\phi\|_{V} \leq 1}\left\langle\rho^{k} P_{k} u^{k-1} \otimes u_{n}^{k-1}, \nabla \phi\right\rangle \\
\leq & \sup _{\|\phi\|_{V} \leq 1}\left\|\rho^{k}\right\|_{L^{\infty}}\left\|P_{k} u^{k-1}\right\|_{L^{4}}\left\|u_{n}^{k}\right\|_{L^{4}}\|\phi\|_{V} \leq C\left\|\rho^{k}\right\|_{L^{\infty}}\left\|\nabla u^{k-1}\right\|\left\|\nabla u_{n}^{k}\right\| \\
\leq & C\left\|\nabla u^{k-1}\right\|^{2}+C\left\|\nabla u_{n}^{k}\right\|^{2} .
\end{aligned}
$$

Portanto,

$$
\begin{aligned}
& \left\|\frac{\partial}{\partial t}\left(\rho^{k} u_{n}^{k}\right)\right\|_{V^{*}} \leq C\|f\|+2 \mu_{r}\left\|w_{n}^{k}\right\|+C\left\|\nabla u_{n}^{k}\right\|+\frac{C}{k}\left\|\Delta \rho^{k}\right\|\left\|\nabla u_{n}^{k}\right\| \\
& +C\left\|\nabla u^{k-1}\right\|^{2}+C\left\|\nabla u_{n}^{k}\right\|^{2} \text {. }
\end{aligned}
$$

Logo,

$$
\int_{0}^{T}\left\|\frac{\partial}{\partial t}\left(\rho^{k} u_{n}^{k}\right)\right\|_{V^{*}} \mathrm{~d} t \leq C(k),
$$

donde $C(k)$ independe de $n$, portanto temos que

$$
\frac{\partial}{\partial t}\left(\rho^{k} u_{n}^{k}\right) \in L^{1}\left(0, T ; V^{*}\right)
$$

uniformemente em $n$.

Passando ao limite quando $n \mapsto \infty$, temos

$$
\frac{\partial}{\partial t}\left(\rho^{k} u_{n}^{k}\right) \mapsto \frac{\partial}{\partial t}\left(\rho^{k} u^{k}\right) \text { fracamente em } L^{1}\left(0, T ; V^{*}\right)
$$

Analogamente, obtemos a mesma estimativa para $\frac{\partial}{\partial t}\left(\rho^{k} w_{n}^{k}\right)$ e, assim, obtém-se

$$
\frac{\partial}{\partial t}\left(\rho^{k} w_{n}^{k}\right) \in L^{1}\left(0, T ; V^{*}\right) \text { uniformemente em } n .
$$

Logo,

$$
\frac{\partial}{\partial t}\left(\rho^{k} w_{n}^{k}\right) \mapsto \frac{\partial}{\partial t}\left(\rho^{k} w^{k}\right) \text { fracamente em } L^{1}\left(0, T ; V^{*}\right)
$$

(ii) Continuidade das funções

Para provar que as funções $u^{k}:[0, T] \mapsto H$ e $w^{k}:[0, T] \mapsto L^{2}(\Omega)^{3}$ são contínuas, tomamos o produto interno no $L^{2}(\Omega)$ na equação $(3.4) \operatorname{com}\left(v-u^{k}\right)$, onde $u^{k} \in V_{k}=\left\langle v_{1}, \ldots, v_{k}\right\rangle$ ( $V_{k}$ é subespaço de dimensão finita em $V$ ) e $v \in$ 
$L^{2}(0, T ; V)$. Analogamente tomamos o produto na equação $(3.5) \operatorname{com}\left(h-w^{k}\right)$, onde $h \in L^{1}\left(0, T ; H_{0}^{1}(\Omega)^{3}\right)$ e $w^{k} \in W_{k}=\left\langle w_{1}, \ldots, w_{k}\right\rangle$ subespaço de dimensão finita em $\left(H_{0}^{1}(\Omega)\right)^{3}$ e $\left\{w_{1}, \ldots, w_{k}, \ldots\right\}$ base de Faedo-Galerkin. Obtém-se

$$
\begin{aligned}
& \left(\rho^{k} \frac{\partial u^{k}}{\partial t}, v-u^{k}\right)+\left(\rho^{k}\left(P_{k} u^{k-1} \cdot \nabla\right) u^{k}, v-u^{k}\right)+\left(\mu+\mu_{r}\right) a\left(u^{k}, v-u^{k}\right) \\
& -2 \mu_{r}\left(\operatorname{rot} w^{k}, v-u^{k}\right)+\frac{1}{2 k}\left(\Delta \rho^{k} u^{k}, v-u^{k}\right)=\left(\rho^{k} f, v-u^{k}\right) \\
& \frac{\partial \rho^{k}}{\partial t}+\left(P_{k} u^{k-1} \cdot \nabla\right) \rho^{k}=\frac{1}{k} \Delta \rho^{k} \\
& \left(\rho^{k} \frac{\partial w^{k}}{\partial t}, h-w^{k}\right)+\left(\rho^{k}\left(P_{k} u^{k-1} \cdot \nabla\right) w^{k}, h-w^{k}\right)+\nu a\left(w^{k}, v-w^{k}\right) \\
& +\gamma\left(\operatorname{div}\left(w^{k}\right), \operatorname{div}\left(h-w^{k}\right)\right)+4 \mu_{r}\left(w^{k}, h-w^{k}\right)-2 \mu_{r}\left(\operatorname{rot} u^{k}, h-w^{k}\right) \\
& +\frac{1}{2 k}\left(\Delta \rho^{k} w^{k}, h-w^{k}\right)=\left(\rho^{k} g, h-w^{k}\right) .
\end{aligned}
$$

Multiplicando a equação (3.25) por $u^{k}\left(v-u^{k}\right)$ e integrando sobre $\Omega \times(0, T)$ esta última expressão, integramos a equação (3.24) com relação a $t$ e, finalmente, somando estas duas desigualdades obtém-se

$$
\begin{aligned}
& \int_{0}^{T}\left\{\left(\frac{\partial}{\partial t}\left(\rho^{k} u^{k}\right), v-u^{k}\right)+\left(\rho^{k}\left(P_{k} u^{k-1} \cdot \nabla\right) u^{k}, v-u^{k}\right)+\left(\left(P_{k} u^{k-1} \cdot \nabla\right) \rho^{k} u^{k}, v-u^{k}\right)\right. \\
& \left.\quad+\left(\mu+\mu_{r}\right) a\left(u^{k}, v-u^{k}\right)-2 \mu_{r}\left(\operatorname{rot} w^{k}, v-u^{k}\right)-\frac{1}{2 k}\left(\Delta \rho^{k} u^{k}, v-u^{k}\right)\right\} \mathrm{d} t \\
& =\int_{0}^{T}\left(\rho^{k} f, v-u^{k}\right) \mathrm{d} t .
\end{aligned}
$$

Somando e restando na última igualdade $\int_{0}^{T}\left(\frac{\partial \varphi^{k} v}{\partial t}, v-u^{k}\right) \mathrm{d} t$, obtemos

$$
\begin{aligned}
& \int_{0}^{T}\left\{\left(\frac{\partial \rho^{k} v}{\partial t}, v-u^{k}\right)+\left(\rho^{k}\left(P_{k} u^{k-1} \cdot \nabla\right) u^{k}, v-u^{k}\right)\left(\left(\rho_{k} u^{k-1} \cdot \nabla\right) u^{k}\left(v-u^{k}\right)\right)\right. \\
& \left.\quad+\left(\mu+\mu_{r}\right) a\left(u^{k}, v-u^{k}\right)-2 \mu_{r}\left(\operatorname{rot} w^{k}, v-u^{k}\right)-\frac{1}{2 k}\left(\Delta \rho^{k} u^{k}, v-u^{k}\right)\right\} \mathrm{d} t \\
& =\int_{0}^{T}\left\{\left(\rho^{k} f, v-u^{k}\right)+\left(\frac{\partial \rho^{k}\left(v-u^{k}\right)}{\partial t}, v-u^{k}\right)\right\} \mathrm{d} t \\
& =\int_{0}^{T}\left(\rho^{k} f, v-u^{k}\right)+\int_{0}^{T}\left(\rho \frac{\partial\left(v-u^{k}\right)}{\partial t}, v-u^{k}\right) \mathrm{d} t
\end{aligned}
$$




$$
\begin{aligned}
& +\frac{1}{2} \int_{0}^{T}\left(\left(v-u^{k}\right) \frac{\partial \rho^{k}}{\partial t}, v-u^{k}\right) \mathrm{d} t+\frac{1}{2} \int_{0}^{T}\left(\left(v-u^{k}\right) \frac{\partial \rho^{k}}{\partial t}, v-u^{k}\right) \mathrm{d} t \\
= & \frac{1}{2} \int_{0}^{T} \frac{\mathrm{d}}{\mathrm{d} t}\left\|\left(\rho^{k}\right)^{1 / 2}\left(v-u^{k}\right)\right\|^{2} \mathrm{~d} t+\frac{1}{2} \int_{0}^{T}\left(\left(v-u^{k}\right) \frac{\partial \rho^{k}}{\partial t}, v-u^{k}\right) \mathrm{d} t \\
& +\int_{0}^{T}\left(\rho^{k} f, v-u^{k}\right) \mathrm{d} t \\
= & \int_{0}^{T}\left(\rho^{k} f, v-u^{k}\right) \mathrm{d} t+\frac{1}{2} \int_{0}^{t} \frac{\mathrm{d}}{\mathrm{d} t}\left\|\left(\rho^{k}\right)^{1 / 2}\left(v-u^{k}\right)\right\|^{2} \mathrm{~d} t \\
& -\frac{1}{2} \int_{0}^{T}\left(\operatorname{div}\left(\rho^{k} P_{k} u^{k-1}\right)\left(v-u^{k}\right), v-u^{k}\right) \mathrm{d} t \\
+ & \frac{1}{2 k} \int_{0}^{T}\left(\left(v-u^{k}\right) \Delta \rho^{k}, v-u^{k}\right) \mathrm{d} t,
\end{aligned}
$$

depois de ter usado a equação (3.25) multiplicada por $\left(v-u^{k}\right)$.

Usando o teorema da divergência, temos

$$
\begin{aligned}
& \left(\rho^{k}\left(P_{k} u^{k-1} \cdot \nabla\right) u^{k}, v-u^{k}\right)+\left(\left(P_{k} u^{k-1} \cdot \nabla\right) \rho^{k}, u^{k}\left(v-u^{k}\right)\right) \\
& =-\left(\rho^{k} P_{k} u^{k-1} \cdot \nabla\left(v^{k}-u^{k}\right), u^{k}\right)
\end{aligned}
$$

$\mathrm{e}$

$$
\begin{aligned}
& -\left(v \operatorname{div}\left[\rho^{k} P_{k} u^{k-1}\right], v-u^{k}\right)+\frac{1}{2}\left(\operatorname{div}\left[\rho^{k} P_{k} u^{k-1}\right]\left(v-u^{k}\right), v-u^{k}\right) \\
& -\left(\rho^{k} P_{k} u^{k-1} \cdot \nabla\left(v-u^{k}\right), u^{k}\right)=\left(\rho^{k} P_{k} u^{k-1} \cdot \nabla v, v-u^{k}\right) .
\end{aligned}
$$

Substituindo a equação (3.25) multiplicada por $v\left(v-u^{k}\right)$ na igualdade (3.27) e usando (3.28), (3.29), temos

$$
\begin{aligned}
& \int_{0}^{T}\left(\rho^{k} \frac{\partial v}{\partial t}, v-u^{k}\right)+\left(\rho^{k} P_{k} u^{k-1} \cdot \nabla v, v-u^{k}\right)+\left(\mu+\mu_{r}\right) a\left(u^{k}, v-u^{k}\right) \\
& -2 \mu_{r}\left(\operatorname{rot} w^{k}, v-u^{k}\right)+\frac{1}{2 k}\left(\Delta \rho^{k} v, v-u^{k}\right)-\left(\rho^{k} f, v-u^{k}\right) \\
& =\frac{1}{2}\left\|\left(\rho^{k}(T)\right)^{1 / 2}\left(v(T)-u^{k}(T)\right)\right\|^{2}-\frac{1}{2}\left\|\left(\rho^{k}(0)\right)^{1 / 2}\left(v(0)-u^{k}(0)\right)\right\|^{2} \\
& \forall v \in L^{2}(0, T ; V) .
\end{aligned}
$$

Similarmente, para a equação (3.26), temos

$$
\int_{0}^{T}\left\{\left(\rho^{k} \frac{\partial h}{\partial t}, h-w^{k}\right)+\left(\rho^{k} P_{k} u^{k-1} \cdot \nabla h, h-w_{k}\right)+\nu a\left(w^{k}, h-w^{k}\right)\right.
$$




$$
\begin{aligned}
& +\gamma\left(\operatorname{div} w^{k}, \operatorname{div}\left(h-w^{k}\right)\right)+\frac{1}{2 k}\left(\Delta \rho^{k} h, h-w^{k}\right)-\left(\rho^{k} g, h-w^{k}\right) \\
& =\frac{1}{2}\left\|\left(\rho^{k}(T)\right)^{1 / 2}\left(h(T)-w^{k}(T)\right)\right\|^{2}-\frac{1}{2}\left\|\left(\rho^{k}(0)\right)^{1 / 2}\left(h(0)-w^{k}(0)\right)\right\|^{2} .
\end{aligned}
$$

Seja $u_{n}^{k}$ solução da equação

$$
\left\{\begin{aligned}
\eta \frac{\partial}{\partial t} u_{\eta}^{k}+u_{\eta}^{k} & =u^{k} \\
u_{\eta}^{k}(0) & =u_{0}^{k}
\end{aligned}\right.
$$

Escolhendo $v=u_{\eta}^{k}$ em (3.30), obtém-se

$$
\begin{aligned}
& -\eta \int_{0}^{t}\left|\left(\rho^{k}\right)^{1 / 2} \frac{\partial u^{k}}{\partial t}\right|^{2} \mathrm{~d} t+\int_{0}^{t}\left\{\rho^{k}\left(u_{\eta}^{k}-u^{n}\right)\left(P_{k} u^{k-1} \cdot \nabla\right) u_{n}^{k}\left(\mu+\mu_{r}\right) a\left(u^{k}, u_{\eta}^{k}-u^{k}\right)\right. \\
& -2 \mu_{r}\left(\operatorname{rot} w^{k}, u_{\eta}^{k}-u^{k}\right)+\frac{1}{2 k}\left(\Delta \rho^{k} u_{\eta}^{k}, u_{\eta}^{k}-u^{k}\right)-\left(\rho^{k} f, u_{\eta}^{k}-u^{k}\right) \\
& =\frac{1}{2}\left\|\left(\rho^{k}(t)\right)^{1 / 2}\left(u_{\eta}^{k}(t)-u^{k}(t)\right)\right\|^{2} .
\end{aligned}
$$

Portanto,

$$
\begin{aligned}
& \left\|u_{\eta}^{k}(t)-u^{k}(t)\right\|^{2} \leq C \int_{0}^{t}\left\{\rho^{k}\left(u_{\eta}^{k}-u^{k}\right)\left(P_{k} u^{k-1}\right) \cdot \nabla u_{\eta}^{k}+\left(\mu+\mu_{r}\right) a\left(u^{k}, u_{\eta}^{k}-u^{k}\right)\right. \\
& -2 \mu_{r}\left(\operatorname{rot} w^{k}, u_{\eta}^{k}-u^{k}\right)+\frac{1}{2 k}\left(\Delta \rho^{k} u_{\eta}^{k}, u_{\eta}^{k}-u^{k}\right)-\left(\rho^{k} f, u_{\eta}^{k}-u^{k}\right)
\end{aligned}
$$

Observamos que o lado direito desta desigualdade tende para zero quando $\eta$ tende para zero. Portanto, $u^{k} \in C([0, T] ; H)$. Similarmente, usando este argumento na equação $(3.31)$, obtemos $w^{k} \in C\left([0, T] ; H_{0}^{1}(\Omega)^{3}\right)$.

\section{(iii) Propriedade reprodutiva}

Assumamos que $\rho^{k}(0)=\rho^{k}(T)$ e definamos

$$
\begin{aligned}
& G=\left\{v \in L^{2}(\Omega)^{3} \mid v=\left(\rho_{0}\right)^{1 / 2} u, u \in V_{k}\right\} \\
& F=\left\{h \in L^{2}(\Omega) \mid h=\left(\rho_{0}\right)^{1 / 2} w, w \in W_{k}\right\} .
\end{aligned}
$$

Observemos que $G, F$ são convexos, fechados e limitados no $L^{2}(\Omega)^{3}$. Queremos provar que a aplicação

$$
S: G \times F \mapsto G \times F
$$


definida por

$$
\left(\rho_{0}^{1 / 2} u_{0}^{k}, \rho_{0}^{1 / 2} w_{0}^{k}\right) \mapsto\left(\left(\rho_{0}\right)^{1 / 2} u^{k}(T),\left(\rho_{0}\right)^{1 / 2} w^{k}(T)\right)
$$

tem um ponto fixo.

Primeiro mostramos que $S$ é contínua. Sejam

$$
\tilde{u}_{n}^{k}(x, t)=\sum_{i=1}^{n} \tilde{c}_{\text {in }}(t) \varphi_{i}(x), \tilde{w}_{m}^{k}(x, t)=\sum_{i=1}^{n} \tilde{d}_{\text {in }}(t) \phi_{i}(x)
$$

soluções do problema $(3.5)$ - (3.7) com dados iniciais $\tilde{u}_{n 0}^{k}, \tilde{w}_{n 0}^{k}$. Analogamente, como foi provada a estimativa (3.18), obtém-se

$$
\begin{aligned}
& \frac{\mathrm{d}}{\mathrm{d} t}\left(\left\|\sqrt{\rho^{k}}\left(u_{n}^{k}-\tilde{u}_{n}^{h}\right)\right\|^{2}+\left\|\sqrt{\rho^{k}}\left(w_{n}^{k}-\tilde{w}_{n}^{k}\right)\right\|^{2}+2 \mu\left\|\nabla\left(u_{n}^{k}-\tilde{u}_{n}^{k}\right)\right\|^{2}+c_{1} \| \nabla\left(w_{n}^{k}-w_{n}^{k} \|^{2}\right.\right. \\
& +c_{2}\left\|\operatorname{div}\left(w_{n}^{k}-\tilde{w}_{n}^{k}\right)\right\|^{2} \leq 0
\end{aligned}
$$

Integrando com respeito a $t$, de 0 a $T$, obtemos

$$
\begin{aligned}
& \left\|\left(\rho^{k}(T)\right)^{1 / 2}\left(u_{n}^{k}(T)-\tilde{u}_{n}^{k}(T)\right)^{2}\right\|^{2}+\left\|\sqrt{\rho^{k}(T)}\left(w_{n}^{k}(T)-\tilde{w}_{n}^{k}(T)\right)\right\|^{2} \\
\leq & C\left(\left\|\sqrt{\rho^{k}(0)}\left(u_{n}^{k}(0)-\tilde{u}_{n}^{k}(0)\right)\right\|^{2}+\left\|\sqrt{\rho^{k}(0)}\left(u_{n}^{k}(0)-\tilde{w}_{n}^{k}(0)\right)\right\|^{2}\right.
\end{aligned}
$$

o que prova a continuidade da aplicação $S$.

Vamos mostrar agora que existe $B_{R}(0)$ tal que

$$
S\left(B_{R}(0)\right) \subset B_{R}(0)
$$

Como $m \leq \rho^{k}(x, t) \leq M$ e $a(v, v) \geq C\|v\|^{2}$ para todo $v \in H_{0}^{1}(\Omega)$, temos

$$
\frac{\mathrm{d}}{\mathrm{d} t}\left(\left\|\sqrt{\rho^{k}} u_{n}^{k}\right\|^{2}+\left\|\sqrt{\rho^{k}} w_{n}^{k}\right\|^{2}\right)+\frac{2 \mu c}{\beta^{2}}\left\|\sqrt{\rho^{k}} u_{n}^{k}\right\|^{2}+\frac{c_{1} c}{\beta^{2}}\left\|\sqrt{\rho^{k}} w_{n}^{k}\right\|^{2} \leq C\left(\|f\|^{2}+\|g\|^{2}\right) .
$$

Pondo $c_{0}=\min \left\{\frac{2 \mu c}{\beta^{2}}, \frac{c_{1} c}{\beta^{2}}\right\}$, temos

$$
\frac{\mathrm{d}}{\mathrm{d} t}\left(\left\|\sqrt{\rho^{k}} u_{n}^{k}\right\|^{2}+\left\|\sqrt{\rho^{k}} w_{n}^{k}\right\|^{2}\right)+c_{0}\left(\left\|\sqrt{\rho^{k}} u_{n}^{k}\right\|^{2}+\left\|\sqrt{\rho^{k}} w_{n}^{k}\right\|^{2} \leq C\left(\|f\|^{2}+\|g\|^{2}\right)\right.
$$


ou

$$
\begin{aligned}
& e^{c_{0} T}\left(\left\|\sqrt{e^{k}(T)} u_{n}^{k}(T)\right\|^{2}+\left\|\sqrt{\rho^{k}(T)} w_{n}^{k}(T)\right\|^{2}\right) \leq\left\|\sqrt{\rho^{k}(0)} u_{n}^{k}(0)\right\|^{2}+\left\|V \rho^{k}(0) w_{n}^{k}(0)\right\|^{2} \\
& +C\left(\int_{0}^{T} e^{c_{0} T}\left(\|f(\tau)\|^{2}+\|g(\tau)\|^{2}\right) \mathrm{d} \tau .\right.
\end{aligned}
$$

Portanto,

$$
\begin{aligned}
\left\|\sqrt{e^{k}(T)} u_{n}^{k}(T)\right\|^{2}+\left\|\sqrt{e^{k}(T)} w_{n}^{k}(T)\right\|^{2} \leq & e^{-c_{0} T}\left(\left\|\sqrt{\rho^{k}(0)} u_{n}^{k}(0)\right\|^{2}+\left\|\sqrt{e^{k}(0)} w_{n}^{k}(0)\right\|^{2}\right. \\
& +C \int_{0}^{T}\left(\|f(\tau)\|^{2}+\|g(\tau)\|^{2}\right) \mathrm{d} \tau \leq R^{2} .
\end{aligned}
$$

Então, é suficiente considerar

$$
R \leq c \frac{\left(\int_{0}^{T}\left(\|f(\tau)\|^{2}+\|g(\tau)\|^{2}\right) \mathrm{d} \tau\right)}{1-e^{-c_{0} T}} .
$$

Logo, pelo Teorema do ponto fixo de Schauder, $S$ tem um ponto fixo. Evidentemente, este ponto fixo é uma solução reprodutiva aproximada. Assim,

$$
\begin{aligned}
\rho_{0}^{1 / 2} u_{0}^{k} & =\left(\rho_{0}\right)^{1 / 2} u^{k}(T) \\
\rho_{0}^{1 / 2} w_{0}^{k} & =\left(\rho_{0}\right)^{1 / 2} w^{k}(T) .
\end{aligned}
$$

A periodicidade de $\rho$ é provada tomando $u^{k}(0)=u^{k}(T)$ e a aplicação $S$ é agora definida como

$$
\left(\rho^{k}(0)^{1 / 2} u^{k}(0), \rho^{k}(0) u^{k}(0)\right) \rightarrow\left(\rho^{k}(T)^{1 / 2} u^{k}(T),\left(\rho^{k}(T)^{1 / 2} w^{k}(T)\right)\right.
$$

Depois de fazer o procedimento similar, concluímos que $\rho^{k}(0)=\rho^{k}(T)$.

Agora provamos o teorema principal deste trabalho.

\subsection{Demonstração do resultado principal}

Para finalizar a prova do principal teorema deste capítulo, vamos obter as seguintes estimativas:

$$
\begin{aligned}
& \int_{0}^{T}\left|u^{k}(t+h)-u^{k}(t)\right|^{2} \mathrm{~d} t \leq c h^{1 / 2}, \forall h>0, \\
& \int_{0}^{T}\left|u^{k}(t+h)-w^{k}(t)\right|^{2} \mathrm{~d} t \leq c h^{1 / 2}, \quad \forall h>0 .
\end{aligned}
$$


Observemos que multiplicando a equação (3.6) por $u^{k}$ e somando a (3.4), obtém-se

$$
\begin{aligned}
\frac{\partial}{\partial t}\left(\rho^{k} u^{k}\right)= & \rho^{k} f+2 \mu_{r} \operatorname{rot} w^{k}+\frac{2}{2 k} \Delta \rho^{k} u^{k}+\left(\mu+\mu_{r}\right) \Delta u^{k}-\left(\rho^{k} P_{k} u^{k-1} \cdot \nabla\right) u^{k} \\
& -\left(P_{k} u^{k-1} \cdot \nabla\right) \rho^{k} u^{k} .
\end{aligned}
$$

Portanto,

$$
\begin{aligned}
\left(\frac{\partial}{\partial t}\left(\rho^{k} u^{k}\right), v\right)= & \left(\rho^{k} f, v\right)+2 \mu_{r}\left(\operatorname{rot} w^{k}, v\right)-\frac{1}{2 k}\left(\Delta \rho^{k} u^{k}, v\right)-\left(\mu+\mu_{r}\right)\left(\nabla u^{k}, \nabla v\right) \\
& -\left(\rho^{k} P_{k} u^{k-1},\left(u^{k} \cdot \nabla\right) v\right) \\
= & \left(F_{k}, v\right), \quad \forall v \in V .
\end{aligned}
$$

Integrando (3.34) de $t$ a $t+h$ obtém-se

$$
\left(\left(\rho^{k} u^{k}\right)(t+h)-\left(\rho^{k} u^{k}\right)(t), v\right)=\left(\int_{t}^{t+h} \frac{\partial}{\partial t}\left(\rho^{k} u^{k}\right), v\right) \mathrm{d} s=\left(\int_{t}^{t+h} F_{k}(s) \mathrm{d} s, v\right)
$$

Tomando $v=u^{k}(t+h)-u^{k}(t)$ e pondo $u_{h}^{k}(t)=u^{k}(t+h)-u^{k}(t) \quad \mathrm{e}$ $\rho_{h}^{k}(t)=\rho^{k}(t+h)-\rho^{k}(t)$, temos

$$
\left(\left(\rho^{k} u^{k}\right)(t+h)-\left(\rho^{k} u^{k}\right)(t), u_{h}^{k}(t)\right)=\left(\rho^{k}(t+h) u_{n}^{k}(t), u_{h}^{k}(t)\right)+\left(\rho_{h}^{k}(t) u^{k}(t), u_{h}^{k}(t)\right)
$$

e levando em conta a equação (3.35) podemos escrever

$$
\left(\rho^{k}(t+h) u_{h}^{k}(t), u_{h}^{k}(t)\right)=-\left(\rho_{h}^{k}(t) u^{k}(t), u_{h}^{k}(t)\right)+\left(\int_{t}^{t+h} F_{k}(s) \mathrm{d} s, u_{h}^{k}(t)\right) .
$$

O mesmo procedimento pode ser aplicado para $w^{k}$, isto é, multiplicamos (3.4) $w^{k}$ e somamos à equação (3.5). Depois, o resultado multiplicado por $v \in H_{0}^{1}(\Omega)^{3}$ fornece a expressão

$$
\begin{aligned}
& \left(\frac{\partial}{\partial t}\left(\rho^{k} w^{k}\right), v\right)=\left(\rho^{k} g, v\right)-\nu\left(\nabla w^{k}, \nabla v\right)-\gamma\left(\operatorname{div} w^{k}, \operatorname{div}\left(v^{h}\right)\right) \\
& -4 \mu_{r}\left(w^{k}, v\right)+2 \mu_{r}\left(\operatorname{rot} u^{k}, v\right)+\frac{1}{2 k}\left(\Delta \rho^{k} w^{k}, v\right)=\left(G_{k}(t), v\right) .
\end{aligned}
$$

Tomando $v=w^{k}(t+h)-w^{k}(t)$ e trocando $u^{k}$ por $w^{k}$ no procedimento anterior, podemos obter

$$
\left(\rho^{k}(t+h) w_{h}^{k}(t), w_{h}^{k}(t)\right)=-\left(\rho_{h}^{k}(t) w^{k}(t), w_{h}^{k}(t)\right)+\left(\int_{t}^{t+h} G_{k}(s) \mathrm{d} s, w_{h}(t)\right) .
$$


Agora vamos voltar para a expressão (3.36). Observando que da equação da densidade, obtemos

$$
\rho_{h}^{k}(t)=\int_{t}^{t+h} \frac{1}{k} \Delta \rho^{k}(s) \mathrm{d} s-\int_{t}^{t+h}\left(P_{k} u^{k-1}(s) \cdot \nabla\right) \rho^{k}(s) \mathrm{d} s
$$

e multiplicando esta igualdade por $u^{k}(t) v$, obtemos

$$
\begin{aligned}
\left(\rho_{h}^{k}(t) u^{k}(t), v(t)\right)= & \frac{1}{k}\left(\int_{t}^{t+h} \Delta \rho^{k}(s) \mathrm{d} s u^{k}(t), v(t)\right) \\
& +\left(\int_{t}^{t+h}\left(P_{k} u^{k-1}(s) \rho^{k}(s) \mathrm{d} s \nabla u^{k}(t), v(t)\right)\right. \\
& +\left(\int_{t}^{t+h}\left(P_{k} u^{k-1}(s) \rho^{k}(s) \mathrm{d} s u^{k}(t), \nabla v(t)\right) .\right.
\end{aligned}
$$

Logo, pondo $v=u_{h}^{k}(t)$, temos

$$
\begin{aligned}
\left(\rho_{h}^{k}(t) u^{k}(t), u_{h}^{k}(t)\right) \leq & \int_{t}^{t+h} \frac{1}{k}\left\|\Delta \rho^{k}(s)\right\| \mathrm{d} s\left\|u^{k}(t)\right\|_{L^{4}}\left\|u_{h}^{k}(t)\right\|_{L^{4}} \\
& +\int_{t}^{t+h}\left\|P_{k} u^{k-1}(s)\right\|_{L^{4}}\left\|\rho^{k}(s)\right\|_{L^{\infty}} \mathrm{d} s\left\|\nabla u^{k}(t)\right\|_{L^{\infty}}\left\|u_{h}^{k}(t)\right\|_{L^{4}} \\
& +\int_{t}^{t+h}\left\|P_{k} u^{k-1}(s)\right\|_{L^{4}}\left\|\rho^{k}(s)\right\|_{L^{\infty}} \mathrm{d} s\left\|u^{k}(t)\right\|_{L^{4}}\left\|\nabla u_{h}^{k}(t)\right\| .
\end{aligned}
$$

Notemos que

$$
\begin{aligned}
& \int_{t}^{t+h} \frac{1}{k}\left\|\Delta \rho^{k}(s)\right\| \mathrm{d} s\left\|u^{k}(t)\right\|_{L^{4}}\left\|u_{h}^{k}(t)\right\|_{L^{4}} \\
\leq & C\left(\int_{t}^{t+h} \mathrm{~d} s\right)^{1 / 2}\left(\int_{t}^{t+h} \frac{1}{k^{2}}\left\|\Delta \rho^{k}(s)\right\|^{2}\right)^{1 / 2}\left\|\nabla u^{k}(t)\right\|\left\|\nabla u_{h}^{k}(t)\right\| \\
\leq & C h^{1 / 2}\left(\int_{t}^{t+h} \frac{1}{k^{2}}\left\|\Delta \rho^{k}(s)\right\|^{2}\right)^{1 / 2}\left\|\nabla u^{k}(t)\right\|\left\|\nabla u_{h}^{k}(t)\right\|, \\
\leq & C \int_{t}^{t+h}\left\|\nabla u^{k-1}(s)\right\| \mathrm{d} s\left\|\nabla u^{k}(t)\right\|\left\|\nabla u_{h}^{k}(t)\right\| \\
\leq & C h^{1 / 2}\left(\int_{t}^{t+h}\left\|\nabla u^{k-1}(s)\right\|^{2} \mathrm{~d} s\right)^{1 / 2}\left\|\nabla u^{k}(t)\right\|\left\|\nabla u_{h}^{k}(t)\right\|
\end{aligned}
$$


e

$$
\begin{aligned}
& \int_{t}^{t+h}\left\|P_{k} u^{k-1}(s)\right\|_{L^{4}}\left\|\rho^{k}(0)\right\|_{L^{\infty}} \mathrm{d} s\left\|u^{k}(t)\right\|_{L^{4}}\left\|\nabla u_{h}^{k}(t)\right\| \\
\leq & C h^{1 / 2}\left(\int_{t}^{t+h}\left\|\nabla u^{k-1}(s)\right\|^{2} \mathrm{~d} s\right)^{1 / 2}\left\|\nabla u^{k}(t)\right\|\left\|\nabla u_{h}^{k}(t)\right\| .
\end{aligned}
$$

Portanto, obtém-se

$$
\begin{aligned}
\left(\rho_{h}^{k}(t) u^{k}(t), u_{h}^{k}(t)\right) \leq & C h^{1 / 2}\left(\left(\int_{t}^{t+h} \frac{1}{k^{2}}\left\|\Delta \rho^{k}(s)\right\|^{2} \mathrm{~d} s\right)^{1 / 2}+\left(\int_{t}^{t+h}\left\|\nabla u^{k-1}(s)\right\|^{2} \mathrm{~d} s\right)^{1 / 2}\right) \\
& \left\|\nabla u^{k}(t)\right\|\left\|\nabla u_{n}^{k}(t)\right\| .
\end{aligned}
$$

Agora passamos a estimar o segundo termo do lado direito da igualdade (3.36), $\operatorname{com} v=u_{h}^{k}(t)$.

$$
\begin{aligned}
& \left(\int_{t}^{t+h} F_{k}(s) \mathrm{d} s, u_{h}^{k}(t)\right) \\
= & \left(\int_{t}^{t+h} \rho^{k}(s) f(s) \mathrm{d} s, u_{h}^{k}(t)\right)+2 \mu_{r}\left(\int_{t}^{t+h} \operatorname{rot} w^{k}(s) \mathrm{d} s, u_{h}^{k}(t)\right) \\
& -\frac{1}{2 k}\left(\int_{t}^{t+h} \Delta \rho^{k}(s) u^{k}(s) \mathrm{d} s, u_{h}^{k}(t)\right)-\left(\mu+\mu_{r}\right)\left(\int_{t}^{t+h} \nabla u^{k}(s) \mathrm{d} s, \nabla u_{h}^{k}(t)\right) \\
& -\int_{t}^{t+h}\left(\rho^{k}(s) P_{k} u^{k-1}(s),\left(u^{k}(s) \cdot \nabla\right) u_{h}^{k}(t)\right) \mathrm{d} s .
\end{aligned}
$$

Estimando cada termo desta última expressão, temos o seguinte

$$
\begin{aligned}
& \left(\int_{t}^{t+h} \rho^{k}(s) f(s) \mathrm{d} s, u_{h}^{k}(t)\right) \leq \int_{t}^{t+h}\left\|\rho^{k}(s)\right\|_{L^{\infty}}\|f(s)\|\left\|u_{h}^{k}(t)\right\| \mathrm{d} s \\
& \leq c h^{1 / 2}\left(\int_{t}^{t+h}\|f(s)\|^{2} \mathrm{~d} s\right)^{1 / 2}\left\|\nabla u_{h}^{k}(t)\right\| \leq c h^{1 / 2}\left\|\nabla u_{h}^{k}(t)\right\| \nabla u_{n}(t) \|
\end{aligned}
$$

e, além disso,

$$
\begin{aligned}
& 2 \mu_{r}\left(\int_{t}^{t+h} \operatorname{rot} w^{k}(s) \mathrm{d} s, u_{h}^{k}(t)\right)=2 \mu_{r}\left(\int_{t}^{t+h} w^{k}(s) \mathrm{d} s, \operatorname{rot} u_{h}^{k}(t)\right) \\
& \leq c h^{1 / 2}\left(\int_{t}^{t+h}\left\|w^{k}(s)\right\|^{2} \mathrm{~d} s\right)^{1 / 2}\left\|\nabla u_{h}^{k}(t)\right\| \leq c h^{1 / 2}\left\|\nabla u_{h}^{k}(t)\right\|, \\
& \left(\mu+\mu_{r}\right)\left(\int_{t}^{t+h} \nabla u^{k}(s) \mathrm{d} s, \nabla u_{h}^{k}(t)\right) \leq c h^{1 / 2}\left(\int_{t}^{t+h} \nabla u^{k}(s) \|^{2} \mathrm{~d} s\right)^{1 / 2}\left\|\nabla u_{h}^{k}(t)\right\| \\
& \leq c h^{1 / 2}\left\|\nabla u_{h}^{k}(t)\right\|
\end{aligned}
$$


e finalmente limitamos o termo da forma seguinte

$$
\begin{aligned}
& \left.\int_{t}^{t+h}\left(\rho^{k}(s) P_{k} u^{k-1}(s), u^{k}(s) \cdot \nabla\right) u_{h}^{k}(t)\right) \mathrm{d} s \\
& \leq\left(\int_{t}^{t+h}\left\|\rho^{k}(0)\right\|_{L^{\infty}}\left\|P_{k} u^{k-1}(s)\right\|_{L^{4}}\left\|u^{k}(s)\right\|_{L^{4}} \mathrm{~d} s\right)\left\|\nabla u_{h}^{k}(t)\right\| \\
& \leq C\left(\int_{t}^{t+h}\left\|\nabla u^{k-1}(s)\right\|\left\|\nabla u^{k}(s)\right\| \mathrm{d} s\right)\left\|\nabla u_{h}^{k}(t)\right\| \\
& \leq C h^{1 / 2}\left(\int_{t}^{t+h}\left(\left\|\nabla u^{k-1}(s)\right\|^{2}+\left\|\nabla u^{k}(0)\right\|^{2}\right) \mathrm{d} s\right)^{1 / 2} \cdot\left\|\nabla u_{h}^{k}(t)\right\| \\
& \leq c h^{1 / 2}\left\|\nabla u_{h}^{k}(t)\right\| .
\end{aligned}
$$

Portanto, concluímos que

$$
\left(\int_{t}^{t+h} F_{k}(s) \mathrm{d} s, u_{h}^{k}(s)\right) \leq c h^{1 / 2}\left\|\nabla u_{h}^{k}(t)\right\| .
$$

Assim,

$$
\left(\rho^{k}(t+h) u_{h}^{k}(t), u_{h}^{k}(t)\right) \leq C h^{1 / 2} \mid \nabla u_{h}^{k}(t)\left\|+C h^{1 / 2}\right\| \nabla u^{k}(t)\|\| \nabla u_{h}^{k}(t) \|
$$

ou

$$
\left\|\left(\rho^{k}\right)^{1 / 2} u_{h}^{k}(t)\right\|^{2} \leq C h^{1 / 2}\left\|\nabla u_{h}^{k}(t)\right\|+C h^{1 / 2}\left\|\nabla u^{k}(t)\right\|\left\|\nabla u_{h}^{k}(t)\right\|
$$

Como $\rho^{k} \geq \alpha>0$, obtém-se que

$$
\left\|u_{h}^{k}(t)\right\|^{2} \leq C h^{1 / 2}\left\|u_{h}^{k}(t)\right\|_{V}\left(1+\left\|u^{k}(t)\right\|_{V}\right)
$$

Integrando esta última desigualdade entre 0 e $T-h$, obtemos

$$
\int_{0}^{T-h}\left\|u_{h}^{k}(t)\right\|^{2} \mathrm{~d} t \leq C h^{1 / 2} \int_{0}^{T-h}\left\|u_{h}^{k}(s)\right\|_{V}\left(1+\left\|u^{k}(s)\right\|_{V}\right) \mathrm{d} s \leq C h^{1 / 2} \int_{0}^{T} g(s) \mathrm{d} s .
$$

Como $g \in L^{1}(0, T)$, conseqüentemente,

$$
u^{k} \in N^{1 / 4,2}(0, T, H) .
$$

Da expressão (3.37), fazendo o mesmo procedimento realizado para $u^{k}$, obtemos

$$
w^{k} \in N^{1 / 4,2}\left(0, T ; L^{2}(\Omega)^{3}\right)
$$


Para fazer a passagem ao limite da formulação variacional do problema aproximado para a formulação variacional do problema contínuo é necessário obter as seguintes convergências abaixo.

Devido a (3.20) e (3.21), obtém-se

$$
\begin{aligned}
u^{k} & \rightarrow u \text { fraco em } L^{2}(0, T ; V), \\
u^{k} & \rightarrow u \text { fraco * em } L^{\infty}(0, T ; H), \\
w^{k} & \rightarrow w \text { fraco em } L^{2}\left(0, T ; H_{0}^{1}(\Omega)^{3}\right), \\
w^{k} & \rightarrow w \text { fraco * em } L^{\infty}\left(0, T ; L^{2}(\Omega)^{3}\right) .
\end{aligned}
$$

Devido à imersão de Sobolev,

$$
V \hookrightarrow\left(L^{q}(\Omega)\right)^{3}
$$

é compacta com $q<q^{*}=6$ e, além disso, a imersão $L^{q}(\Omega)^{3} \hookrightarrow L^{2}(\Omega)^{3}$ é contínua.

Devido ao Lema 1.4, temos

$$
L^{2}(0, T ; V) \cap N^{1 / 2,2}\left(0, T ; L^{2}(\Omega)^{3}\right) \hookrightarrow L^{2}\left(0, T ; L^{q}(\Omega)^{3}\right)
$$

$\operatorname{com} q \in[2,6)$ é compacta e portanto

$$
u^{k} \rightarrow u \text { na topologia forte de } L^{2}\left(0, T ; L^{q}(\Omega)^{3}\right) .
$$

Similarmente, como a imersão de Sobolev $H_{0}^{1}(\Omega)^{3} \hookrightarrow L^{q}(\Omega)^{3}$ com $q \in[2,6)$ é compacta, obtém-se

$$
w^{k} \mapsto w \text { na topologia forte de } L^{2}\left(0, T ; L^{q}(\Omega)^{3}\right) \operatorname{com} q \in[2,6) .
$$

Para $P_{k} u^{k-1}$ valem as mesmas estimativas que para $u^{k-1}$. Temos

$$
\begin{aligned}
& P_{k} u^{k-1} \rightarrow u \text { na topologia fraca de } L^{2}(0, T ; V), \\
& P_{k} u^{k-1} \mapsto u \text { na topologia forte de } L^{2}\left(0, T ; L^{q}(\Omega)^{3}\right) .
\end{aligned}
$$


Além disso,

$$
\rho^{k} \mapsto \rho \text { na topologia fraca }{ }^{*} \text { de } L^{\infty}\left(Q_{T}\right)
$$

Observe-se que

$$
\begin{aligned}
\left(P_{k} u^{k-1} \rho^{k}, \varphi\right)-(\rho u, \varphi) & =\left(\rho^{k} u, \varphi\right)-(\rho u, \varphi)+\left(\rho^{k}\left(P_{k} u^{k-1}\right), \varphi\right)-\left(\rho^{k} u, \varphi\right) \\
& =\left(\left(\rho^{k}-\rho\right) u, \varphi\right)+\left(\rho^{k}\left(P_{k} u^{k-1}-u\right), \varphi\right) .
\end{aligned}
$$

Vemos daqui que quando $k \mapsto \infty$, os termos da última expressão tende para zero devido à convergência fraca ${ }^{*}$ de $\rho^{k} \mapsto \rho$ e, no segundo termo, devido à limitação uniforme de $\rho^{k}$ em $L^{\infty}(Q)$ e a convergência forte de $u^{k}$ a $u$ em $L^{2}\left(0, T ; L^{q}(\Omega)^{3}\right)$. Portanto,

$$
\left(P_{k} u^{k-1}\right) \rho^{k} \rightarrow \rho u \text { na topologia fraca de } L^{2}\left(0, T ; L^{q}(\Omega)^{3}\right)
$$

Agora faremos o passo ao limite, desde a Definição 3.3 à Definição 3.1, por exemplo,

$$
\begin{aligned}
\lim _{k \rightarrow \infty} \int_{0}^{T}\left(\frac{\partial}{\partial t}\left(\rho^{k} u^{k}\right), \varphi\right) \mathrm{d} t & =\int_{0}^{T}\left(\frac{\partial}{\partial t}(\rho u), \varphi\right) \mathrm{d} t \\
& =-\int_{0}^{T}\left(\rho u, \frac{\partial \varphi}{\partial t}\right) \mathrm{d} t
\end{aligned}
$$

e

$$
\lim _{k \mapsto \infty} \int_{0}^{T}\left(\rho^{k} P_{k} u^{k-1},\left(u^{k} \cdot \nabla\right) \varphi\right)=\int_{0}^{T}(\rho u,(u \cdot \nabla) \varphi),
$$

devido a (3.49) e (3.44). De forma similar, obtemos para as $w^{k}$,

$$
\lim _{k \mapsto \infty} \int_{0}^{T}\left(\rho^{k} P_{k} u^{k-1},\left(w^{k} \cdot \nabla\right) \psi\right) \mathrm{d} t=\int_{0}^{T}(\rho u, w \cdot \nabla \psi) \mathrm{d} t,
$$

devido a (3.49) e (3.45) e

$$
\begin{aligned}
\lim _{k \mapsto \infty} \int_{0}^{T}\left(\frac{\partial}{\partial t}\left(\rho^{k} w^{k}\right), \psi\right) \mathrm{d} t & =\int_{0}^{T}\left(\frac{\partial}{\partial t}(\rho w), \psi\right) \mathrm{d} t \\
& =-\int_{0}^{T}\left(\rho w, \frac{\partial \psi}{\partial t}\right) \mathrm{d} t
\end{aligned}
$$


Vejamos agora os termos abaixo:

$$
\begin{aligned}
\frac{1}{2 k} \int_{0}^{T}\left(\Delta \rho^{k} u^{k}, \varphi\right) \mathrm{d} s & \leq \frac{1}{2 k} \int_{0}^{T}\left\|\Delta \rho^{k} u^{h}\right\|_{L^{2}}\|\varphi\|_{L^{2}} \mathrm{~d} s \\
& \leq \frac{c}{2 k} \int_{0}^{T}\left\|\Delta \rho^{k}\right\|_{L^{2}}\|\varphi\| \mathrm{d} s \\
& \leq \frac{c}{2 k} \int_{0}^{T}\left\|\Delta \rho^{k}\right\|_{L^{2}} \mathrm{~d} s
\end{aligned}
$$

Como

$$
\sup \left\|\rho^{k}\right\|_{H^{1}(\Omega)}+\frac{1}{k} \int_{0}^{T}\|\rho\|_{W^{2,2}(\Omega)}^{2} \mathrm{~d} s \leq C_{2}
$$

obtém-se

$$
\frac{c}{2 k} \int_{0}^{T}\left\|\Delta \rho^{k}\right\|_{L^{2}} \mathrm{~d} s \leq \frac{\tilde{C}}{k} \mapsto 0 \text { quando } k \mapsto \infty
$$

De forma similar temos que o termo

$$
\frac{1}{2 k} \int_{0}^{T}\left(\Delta \rho^{k} w^{k}, \varphi\right) \mathrm{d} s \mapsto 0 \text { quando } k \mapsto \infty
$$

Devido à linearidade dos outros termos, é fácil passar ao limite nas duas expressões variacionais da Definição 3.3 e usando (3.50) - (3.55) vemos que $u, w$ satisfazem as equações (ii) da Definição 3.1.

Consideremos agora a equação

$$
\begin{aligned}
\frac{\partial \rho^{k}}{\partial t}+\nabla \cdot\left(P_{k} u^{k-1} \cdot \rho^{k}\right) & =\frac{1}{k} \Delta \rho^{k}\left(\frac{\partial \rho^{k}}{\partial t}, \varphi\right)_{H^{-1}(\Omega) H_{0}^{1}(\Omega)}+\left(\nabla \cdot\left(P_{k} u^{k-1} \rho^{k}\right), \varphi\right)_{H^{-1}(\Omega) H_{0}^{1}(\Omega)} \\
& =\frac{1}{k}\left(\Delta \rho^{k}, \varphi\right)_{H^{-1}(\Omega) H_{0}^{1}(\Omega)} .
\end{aligned}
$$

Observemos que

$$
\frac{1}{k}\left|\int_{0}^{T}\left(\Delta \rho^{k}, \varphi\right)\right| \leq \frac{1}{k}\left|\int_{0}^{T}\left(\nabla \rho^{k}, \nabla \varphi\right) \mathrm{d} t\right|,
$$

já que $\frac{\partial \rho^{k}}{\partial n}=0$ sobre $\partial \Omega$, portanto

$$
\begin{aligned}
& \frac{1}{k}\left|\int_{0}^{T}\left(\Delta \rho^{k}, \varphi\right)\right| \leq \frac{1}{\sqrt{k}} \int_{0}^{T}\left(\int_{\Omega} \frac{1}{\sqrt{k}}\left|\nabla \rho^{k}\right|^{2} \mathrm{~d} x\right)^{1 / 2}\left(\int_{\Omega}|\nabla \varphi|^{2}\right)^{1 / 2} \\
& \leq \frac{1}{2 \sqrt{k}}\left\{\left.\int_{0}^{T} \frac{1}{k} \int_{\Omega}\left|\nabla \rho^{k} \|^{2} \mathrm{~d} x \mathrm{~d} t+\int_{0}^{T} \int_{\Omega}\right| \nabla \varphi\right|^{2} \mathrm{~d} x \mathrm{~d} t\right\} .
\end{aligned}
$$


Por (3.13) obtém-se

$$
\frac{1}{k}\left|\int_{0}^{T}\left(\Delta \rho^{k}, \varphi\right)\right| \leq \frac{c}{2 \sqrt{k}} \mapsto 0 \text { quando } k \mapsto \infty
$$

Portanto, $\left\{\frac{\partial \rho^{k}}{\partial t}\right\}$ está limitada em $L^{2}\left(0, T ; H^{-1}(\Omega)\right)$, isto é,

$$
\frac{\partial \rho^{k}}{\partial t} \mapsto \chi \text { na topologia fraca de } L^{2}\left(0, T ; H^{-1}(\Omega)\right) .
$$

Observemos, ainda, como $\rho^{k} \mapsto \rho$ na topologia fraca ${ }^{*}$ em $L^{\infty}(Q)$. Temos em particular que

$$
\left\langle\rho^{k}, \varphi\right\rangle \mapsto\langle\rho, \varphi\rangle \text { em } \mathcal{D}^{\prime}((0, T) \times \Omega)
$$

Assim,

$$
\left\langle\frac{\partial \rho^{k}}{\partial t}, \varphi\right\rangle=-\left\langle\rho^{k}, \frac{\partial \varphi}{\partial t}\right\rangle \mapsto-\left\langle\rho, \frac{\partial \varphi}{\partial t}\right\rangle=\left\langle\frac{\partial \rho}{\partial t}, \varphi\right\rangle .
$$

Desta última relação e junto com (3.56), concluímos que

$$
\frac{\partial \rho^{k}}{\partial t} \mapsto \frac{\partial \rho}{\partial t} \text { na topologia fraca de } L^{2}\left(0, T ; H^{-1}(\Omega)\right)
$$

Portanto, da equação (1.3) obtemos, por passo ao limite sobre $k$, a equação

$$
\frac{\partial \rho}{\partial t}+\nabla \cdot(u \rho)=0
$$

Além disso, como $\rho^{k} \in C\left([0, T] ; H^{-1}(\Omega)^{3}\right)$, pois usando o Lema $1.5 \operatorname{com} L^{\infty}(\Omega) \hookrightarrow$ $H^{-1}(\Omega)$, que é compacta, podemos concluir que

$$
\rho^{k}(x, 0) \mapsto \rho(x, 0)=\rho_{0}(x) \text { em } H^{-1}(\Omega)
$$

Assim, finalizamos a prova do resultado principal.

Observação Um problema interessante a estudar é a existência e unicidade de soluções periódicas fortes. Devemos notar que o problema está em aberto mesmo no caso dos fluidos não-homogêneos. 
Para as equações de Navier-Stokes clássicas isto já foi respondido por Serrin [63]. Os argumentos usados por ele são os argumentos de perturbação.

Recentemente Kato [34] mostra resultados melhores que os de Serrin, fazendo uso do método de Galerkin espectral. Em [50] mostram a existência e unicidade de soluções periódicas fortes para uma classe de equações de evolução abstratas, que contém como caso particular as equações de Navier-Stokes clássicas, magnetohidrodinâmica, Boussinesq, magneto-micropolares com densidade constante.

Talvez as idéias desenvolvidas aqui possam ser úteis para responder a questão da existência e unicidade de soluções periódicas fortes. 


\section{Capítulo 4}

\section{Equações variacionais}

\subsection{Introdução}

As desigualdades variacionais (ou inequações variacionais) em mecânica são bem documentadas no livro clássico de Duvaut e Lions [19], Glowinski, Lions e Tremoliéres [29]. Basicamente elas surgem quando colocamos restrições às incógnitas, por exemplo, exigir que o campo de velocidade seja limitado em alguma norma $L_{p}$, o que refletiria algumas limitações físicas.

Nas equações de mecânica dos fluidos existem alguns trabalhos, tanto para o caso estacionário e de evolução, ver por exemplo, Biroli [7], Lions [43], Khoi e Schmitt [35], Lukaszewicz [48], Salvi [71], Antontsev et al. [2].

Binoli estuda as equações de Navier-Stokes de evolução clássicas com a restrição $u(t, x) \geq 0$. Lions nos apresenta uma variante dos fluidos não-homogêneos de evolução, Khoi e Schmitt estudam as bifurcaç̃ões das inequações de Navier-Stokes estacionárias. Lukaszewicz o caso das inequações micropolares (com densidade constante) estacionárias, Antontsev et al. [2] o caso das inequações dos fluidos não-homogêneos de evolução com condições sobre as derivadas normais sobre o campo de velocidade e, finalmente, Salvi também estuda o problema dos fluidos não-homogêneos com condições tipo nulas na fronteira com o campo de velocidade pertencendo a um 
conjunto convexo.

Apresentamos neste capítulo uma extensão dos resultados de Salvi para o modelo dos fluidos não-homogêneos assimétricos de evolução. Estes seriam, em nosso conhecimento, os primeiros resultados desta natureza para as equações micropolares de evolução.

A técnica que utilizaremos são as técnicas clássicas descritas em [19] e [29], a saber, aproximação por problemas finito-dimensionais, introduzindo os operadores de projeção correspondentes que nos permitem reescrever nosso problema original como uma equação. Posteriormente, provaremos algumas estimativas a priori uniformes para as aproximaçẽos, as quais nos permitirão passar ao limite e mostraremos que o limite é de fato uma solução para nosso problema original.

Salientamos que nosso trabalho difere do trabalho de Salvi na obtenção da convergência forte nas aproximações, pois utilizaremos os resultados devidos a J. Simon [80] em espaços de Nikolskii para obter a convergência forte mencionada.

\subsection{Colocação do problema das formulações va- riacionais}

O objetivo desta seção é fazer uma formulação variacional do sistema de equações (0.1) - (0.3) que utilizaremos posteriormente nas seções seguintes. Para isto, começamos definindo os seguintes espaços vetoriais

$$
\begin{aligned}
\Phi= & \left\{v \in L^{2}(0, T ; V) / \frac{\partial v}{\partial t} \in L^{2}\left(0, T ; L^{6 / 5}(\Omega)\right), \frac{\partial v}{\partial x_{i}} \in L^{\infty}\left(0, T ; L^{3 / 2}(\Omega),\right.\right. \\
& i=1,2,3\} \\
\Psi= & \left\{v \in L^{2}\left(0, T ; H^{1}(\Omega)\right) / \frac{\partial v}{\partial t} \in L^{2}\left(0, T ; L^{6 / 5}(\Omega)\right), \frac{\partial v}{\partial x_{i}} \in L^{\infty}\left(0, T ; L^{3 / 2}(\Omega),\right.\right. \\
& i=1,2,3\}
\end{aligned}
$$

Além disso, consideramos os dois conjuntos convexos $\mathbb{K}_{1} \subseteq V$ e $\mathbb{K}_{2} \subseteq H_{0}^{1}(\Omega)$ 
não vazios, contendo o elemento nulo.

Seja. $B(u, v)=\nu(\nabla u, \nabla v)+\gamma(\operatorname{div} u, \operatorname{div} v)$ e consideremos o sistema.

$$
\begin{aligned}
& \left(\rho \frac{\partial u}{\partial t}, v-u\right)+\int_{\Omega} \rho(u \cdot \nabla) u(v-u) \mathrm{d} x+\mu a(u, v-u)+2 \mu_{r}(\operatorname{rot} w, v-u) \\
& \geq(\rho f, v-u) v \in \mathbb{K}_{1} \\
& \left(\rho \frac{\partial w}{\partial t}, \psi-w\right)+\int_{\Omega} \rho(u \cdot \nabla) w(\psi-w) \mathrm{d} x+B(w, \psi-w)+4 \mu_{r}(w, \psi-w) \\
& \geq 2 \mu_{r}(\operatorname{rot} u, \psi-w)+(\rho g, \psi-w) \psi \in \mathbb{K}_{2} \\
& \frac{\partial \rho}{\partial t}+\operatorname{div}(\rho u)=0
\end{aligned}
$$

satisfzendo as condições iniciais

$$
u(x, 0)=u_{0}(x) \text { em } \Omega, \rho(x, 0)=\rho_{0}(x) \text { em } \Omega, w(x, 0)=w_{0}(x) \text { em } \Omega
$$

e as condições de fronteira

$$
u=w=0 \text { sobre } \Sigma_{T}=\partial \Omega \times(0, T)
$$

O objetivo deste capítulo é estudar a existência de soluções fracas para o sistema (4.1) - (4.5) no sentido da formulação variacional que obteremos nos cálculos a seguir. Para isto, multipliquemos por $u \cdot(v-u)$ a equaçãa (4.3) e integrando sobre $Q_{T}=\Omega \times(0, T)$ obtém-se

$$
\int_{0}^{T}\left\{\left(u \frac{\partial \rho}{\partial t}, v-u\right)+((u-\nabla) \rho u, v-u)\right\} \mathrm{d} t=0
$$

Depois integramos a desigualdade (4.1) em relação à variável $t$, de 0 até $T$, e somando o resultado a (4.6) temos

$$
\begin{gathered}
\int_{o}^{T}\left(\rho \frac{\partial}{\partial t}(\rho u), v-u\right)+((u \cdot \nabla) \rho u, v-u)+(\rho u \cdot \nabla u, v-u) \\
+\mu a(u, v-u)+2 \mu_{r}(\operatorname{rot} w, v-u) \\
\geq \quad(\rho f, v-u) \forall v \in \mathbb{K}_{1},
\end{gathered}
$$


onde $v \in \mathbb{K}_{1}$ e lembremos que $a(u, w)=(\nabla u, \nabla w)$.

Observemos que $(u \cdot \nabla u, \rho(v-u))+(u(u \cdot \nabla) \rho, v-u)=-(\rho u, u \cdot \nabla(v-w))$. De fato, temos

$$
\begin{aligned}
\operatorname{div}[u(u \cdot(v-u)) \rho] & =\operatorname{div}(u)(u \cdot(v-u) \rho)+u \cdot \nabla[u \cdot(v-u) \rho] \\
& =u \cdot \nabla[u \cdot(v-u) \rho] \\
& =u \cdot \nabla u(v-u) \rho+u[u \cdot \nabla(v-u) \rho] \\
& =\rho u \cdot \nabla u(v-u)+u[u \cdot \nabla(v-u) \rho]+u[u \cdot(v-u) \nabla \rho] \\
& =\rho u \cdot \nabla u(v-u)+u(u \cdot \nabla(v-u)) \rho+\rho(u \cdot \nabla \rho) u(v-u)
\end{aligned}
$$

Usando o teorema da divergência, obtém-se a afirmação anterior e, portanto, temos

$$
\left\{\begin{array}{l}
\int_{0}^{T}\left(\frac{\partial}{\partial t}(\rho u), v-u\right)-(\rho u, u \cdot \nabla(v-u))+\mu a(u, v-u) \\
+2 \mu_{r}(\operatorname{rot} w, v-u) \geq(\rho f, v-u), \quad \forall v \in \mathbb{K}_{1}
\end{array}\right.
$$

Somemos nesta última expressão a quantidade $\int_{0}^{T}\left(\frac{\partial(\rho v)}{\partial t}, v-u\right) \mathrm{d} t$ e observando que

$$
\begin{aligned}
& \int_{0}^{T}\left(\frac{\partial(\rho u)}{\partial t}, v-u\right)+\left(\frac{\partial \rho v}{\partial t}, v-u\right)-\left(\frac{\partial \rho v}{\partial t}, v-u\right) \mathrm{d} t \\
= & \int_{0}^{T}\left(\frac{\partial \rho(u-v)}{\partial t}, v-u\right) \mathrm{d} t+\int_{0}^{T}\left(\frac{\partial \rho v}{\partial t}, v-u\right) \mathrm{d} t .
\end{aligned}
$$

Substituindo isto na equação (4.8), obtém-se

$$
\begin{aligned}
& \int_{0}^{T}\left(\frac{\partial \rho v}{\partial t}, v-u\right)-(\rho u, u \cdot \nabla(v-u))+\mu a(u, v-u)+2 \mu_{r}(\operatorname{rot} w, v-u) \\
\geq & (\rho f, v-u)+\int_{0}^{T}\left(\frac{\partial \rho(u-v)}{\partial t}, v-u\right) \mathrm{d} t, \quad \forall v \in \mathbb{K}_{1} .
\end{aligned}
$$


Observe-se ainda que

$$
\begin{aligned}
\int_{0}^{T}\left(\frac{\partial \rho(v-u)}{\partial t}, v-u\right) \mathrm{d} t= & \int_{0}^{T}\left(\frac{\rho \partial(v-u)}{\partial t}, v-u\right) \mathrm{d} t+\frac{1}{2} \int_{0}^{T}\left((v-u) \frac{\partial \rho}{\partial t}, v-u\right) \mathrm{d} t \\
& +\frac{1}{2} \int_{0}^{T}\left(\frac{\rho \partial(v-u)}{\partial t}, v-u\right) \mathrm{d} t \\
= & \frac{1}{2} \frac{d}{\mathrm{~d} t} \int_{0}^{T}\left\|\rho^{1 / 2}(v-u)\right\|^{2}+\frac{1}{2} \int_{0}^{T}\left((v-u) \frac{\partial \rho}{\partial t}, v-u\right) \mathrm{d} t
\end{aligned}
$$

Portanto de (4.9) obtém-se

$$
\begin{aligned}
& \left.\int_{0}^{T}\left(\frac{\partial(\rho v)}{\partial t}, v-u\right)-\rho u, u \cdot \nabla(v-u)\right)+\mu a(u, v-u)+2 \mu_{r}(\operatorname{rot} w, v-u) \\
\geq & (\varphi f, v-u)+\frac{1}{2} \frac{d}{\mathrm{~d} t} \int_{0}^{T}\left\|\rho^{1 / 2}(v-u)\right\|^{2}+\frac{1}{2} \int_{0}^{T}\left((v-u) \frac{\partial \rho}{\partial t}, v-u\right) \mathrm{d} t
\end{aligned}
$$

Como $\frac{\partial \rho}{\partial t}+(u \cdot \nabla) \rho=0$ obtemos

(i) $\int_{0}^{T}\left(v \frac{\partial \rho}{\partial t}, v-u\right) \mathrm{d} t=-\int_{0}^{T}((u \cdot \nabla \rho) v, v-u) \mathrm{d} t$

(ii) $\int_{0}^{T}\left((v-u) \frac{\partial \rho}{\partial t}, v-u\right) \mathrm{d} t=-\int_{0}^{T}((v-u) u \cdot \nabla \rho, v-u) \mathrm{d} t$

Substituindo (i), (ii) em (4.10) obtém-se

$$
\begin{aligned}
\int_{0}^{T}\left\{\left(\rho \frac{\partial v}{\partial t}, v-u\right)\right. & -((u \cdot \nabla \rho) v, v-u)+\frac{1}{2}((v-u) \cdot u \cdot \nabla \rho, v-u) \\
& -(\rho u, u \cdot \nabla(v-u))+\mu a(u, v-u)+2 \mu_{r}(\operatorname{rot} w, v-u) \\
& \left.\geq(\rho f, v-u)+\frac{1}{2} \frac{d}{\mathrm{~d} t} \int_{0}^{T}\left\|\rho^{1 / 2}(v-u)\right\|^{2}\right\}
\end{aligned}
$$

Observe-se ainda que

$-(v(u \cdot \nabla \rho), v-u)+\frac{1}{2}((v-u) u \cdot \nabla \rho, v-u)-(\rho u, u \cdot \nabla(v-u))=(\rho u \cdot \nabla u, v-u)$,

já que

$$
\begin{aligned}
\operatorname{div}[(\rho u) v \cdot(v-u)] & =\operatorname{div}(\rho u) v \cdot(v-u)+\rho u \cdot \nabla[v \cdot(v-u)] \\
& =v \operatorname{div}(\rho u) \cdot(v-u)+\rho(u \cdot \nabla v)(v-u)+v(\rho u \cdot \nabla)(v-u) .
\end{aligned}
$$


Usando o Teorema da divergência obtém-se

$$
\begin{aligned}
(v \operatorname{div}(\rho u), v-u) & =-(\rho(u \cdot \nabla v), v-u)-(\rho v, u \cdot \nabla(v-u)) \\
& =-(\rho u \cdot \nabla v, v)+(\rho u \cdot \nabla v, u)-(\rho v, u \cdot \nabla v)+(\rho v, u \cdot \nabla u)
\end{aligned}
$$

vem que

$$
(v \operatorname{div}(\rho u), v-u)=-2(\rho u \cdot \nabla v, v)+(\rho u \cdot \nabla v, u)+(\rho u \cdot \nabla u, u) .
$$

Além disso,

$$
\begin{aligned}
\operatorname{div}[(\rho u)(v-u) \cdot(v-u)] & =\operatorname{div}(\rho u)(v-u) \cdot(v-u)+\rho u \cdot \nabla[(v-u) \cdot(v-u)] \\
& =\operatorname{div}(\rho u)(v-u) \cdot(v-u)+2(v-u)(\rho u \cdot \nabla)(v-u)
\end{aligned}
$$

e usando o teorema da divergêngia novamente, obtém-se

$$
(\operatorname{div}(\rho u) v-u, v-u)=-2(\rho u \cdot \nabla(v-u), v-u)
$$

Logo, obtém-se, usando (4.11) e (4.12)

$$
\begin{aligned}
& \frac{1}{2}((v-u) \operatorname{div}(\rho u), v-u)-(v \operatorname{div}(\rho u), v-u)-(\rho u, u \cdot \nabla(v-u)) \\
= & 2(\rho u \cdot \nabla v, v)+(\rho u \cdot \nabla v, u)-(\rho u \cdot \nabla u, u)-(\rho u \cdot \nabla(v-u), v-u) \\
& -(\rho u, u \cdot \nabla v)+(\rho u, u \cdot \nabla u) \\
= & 2(\rho u \cdot \nabla v, v)-2(\rho u \cdot \nabla u, u)-(\rho u \cdot \nabla u, v)-(\rho u \cdot \nabla(v-u), v) \\
& +(\rho u \cdot \nabla(v-u), u)+(\rho u, u \cdot \nabla u) \\
= & 2(\rho u \cdot \nabla v, v)-2(\rho u \cdot \nabla v, u)-(\rho u \cdot \nabla u, v)-(\rho u \cdot \nabla(v-u), v) \\
& +(\rho u \cdot \nabla(v-u), u)+(\rho u, u \cdot \nabla u) \\
= & 2(\rho u \cdot \nabla v, v)-2(\varphi u \cdot \nabla v, u)-(\rho u \cdot \nabla u, v)-(\rho u \cdot \nabla v, v)+(\rho u \cdot \nabla u, v) \\
& +(\rho u \cdot \nabla v, u)-(\rho u \cdot \nabla u, u)+(\rho u, u \cdot \nabla u)=(\rho u \cdot \nabla v, v)-(\rho u \cdot \nabla v, u) \\
= & (\rho u \cdot \nabla v, v-u)
\end{aligned}
$$


Finalmente obtém-se

$$
\begin{aligned}
& \int_{0}^{T}\left(\rho \frac{\partial v}{\partial t}, v-u\right)+(\rho u \cdot \nabla v, v-u)+\mu a(u, v-u)+2 \mu_{r}(\operatorname{rot} w, v-u) \\
& \geq(\rho f, v-u)+\frac{1}{2} \frac{d}{\mathrm{~d} t} \int_{0}^{T}\left\|\rho^{1 / 2}(v-u)\right\|^{2} \\
& \geq(\rho f, v-u)-\frac{1}{2}\left\|\rho^{1 / 2}(0)(v(0)-u(0))\right\|^{2}
\end{aligned}
$$

com $u \in L^{2}(0, T ; V), u \in \mathbb{K}_{1}, \rho \in L^{\infty}\left(Q_{T}\right), \forall v \in \Phi, v \in \mathbb{K}_{1}$.

Agora faremos a formulação Variacional para a equação (0.3). Temos

$$
\begin{aligned}
& \left(\rho \frac{\partial w}{\partial t}, \phi-w\right)+(\rho(u \cdot \nabla) w, \phi-w)-\beta(\triangle w, \phi-w)-\gamma(\operatorname{div}(u), \operatorname{div}(\phi-w)) \\
& +4 \mu_{r}(w, \phi-w)-2 \mu_{r}(\operatorname{rot}(u), \phi-w) \geq(\rho g, \phi-w) .
\end{aligned}
$$

Multipliquemos a equação (4.3) por $w \cdot(\phi-w)$ e integremos em relação ao tempo, de 0 até $T$. Então

$$
\int_{0}^{T}\left(\frac{\partial \rho}{\partial t} w, \phi-w\right) \mathrm{d} t+\int_{0}^{T}(\operatorname{div}(\rho u) w, \phi-w) \mathrm{d} t=0 .
$$

Integrando em relação a $t$ a desigualdade (4.14) e somando a expressão anterior, obtém-se

$$
\begin{aligned}
& \left\{\int_{0}^{T}\left(\frac{\partial}{\partial t}(\rho w), \phi-w\right)+(\operatorname{div}(\rho u) w, \phi-w)+(\rho(u \cdot \nabla) w, \phi-w)+B(w, \phi-w)\right. \\
& \left.\left.+4 \mu_{r}(w, \phi-w)-2 \mu_{r}(\operatorname{rot} u, \phi-w)\right\}\right\} \mathrm{d} t \\
& \geq \int_{0}^{T}(\rho g, \phi-w) \mathrm{d} t .
\end{aligned}
$$

Observemos que

$$
\begin{aligned}
& \operatorname{div}[u(w \cdot(\phi-w)) \rho]=\operatorname{div}(u) w \cdot(\phi-w) \rho+u \cdot \nabla[w \cdot(\phi-w) \rho] \\
& =u \cdot \nabla[w \cdot(\phi-w) \rho] \\
& =u \cdot \nabla w(\phi-w) \rho+w(u \cdot \nabla(\phi-w) \rho)+w(\phi-w) u \cdot \nabla \rho=0
\end{aligned}
$$

Portanto, pelo teorema da divergência

$$
(\rho(u \cdot \nabla) w, \phi-w)+((u \cdot \nabla \rho) w, \phi-w)=-(\rho w, u \cdot \nabla(\phi-w))
$$


Logo, substituindo em (4.15), obtém-se

$$
\begin{aligned}
\int_{0}^{T}\left\{\left(\frac{\partial}{\partial t}(\rho w), \phi-w\right)\right. & -(\rho w, u \cdot \nabla(\phi-w))+B(w, \phi-w)+4 \mu_{r}(w, \phi-w) \\
& \left.-2 \mu_{r}(\operatorname{rot} u, \phi-w)\right\} \mathrm{d} t \geq \int_{0}^{T}(\rho g, \phi-w) \mathrm{d} t
\end{aligned}
$$

Nesta última expressão vamos somar e restar o termo $\int_{0}^{T}\left(\frac{\partial(\rho \phi)}{\partial t}, \phi-w\right) \mathrm{d} t$

$$
\begin{aligned}
& \int_{0}^{T}\left(\frac{\partial(\rho w)}{\partial t}, \phi-w\right)+\left(\frac{\partial(\rho \phi)}{\partial t}, \phi-w\right)-\left(\frac{\partial(\rho \phi)}{\partial t}, \phi-w\right)-(\rho w, u \cdot \nabla(\phi-w)) \\
& +B(w, \phi-w)+4 \mu_{r}(w, \phi-w)-2 \mu_{r}(\operatorname{rot} u, \phi-w) \\
\geq & \int_{0}^{T}(\rho g, \phi-w) \mathrm{d} t
\end{aligned}
$$

o que implica em

$$
\begin{aligned}
& \int_{0}^{T}\left(\frac{\partial(\rho \phi)}{\partial t}, \phi-w\right)-(\rho w, u \cdot \nabla(\phi-w))+B(w, \phi-w)+4 \mu_{r}(w, \phi-w) \\
& -2 \mu_{r}(\operatorname{rot} u, \phi-w) \\
\geq & \int_{0}^{T}(\rho g, \phi-w) \mathrm{d} t+\int_{0}^{T}\left(\frac{\partial \rho(\phi-w)}{\partial t}, \phi-w\right) \mathrm{d} t
\end{aligned}
$$

Já que

$$
\begin{gathered}
\int_{0}^{T}\left(\frac{\partial \rho(\phi-w)}{\partial t}, \phi-w\right) \mathrm{d} t=\int_{0}^{T}\left(\rho \frac{\partial(\phi-w)}{\partial t}, \phi-w\right) \mathrm{d} t+\frac{1}{2} \int_{0}^{T}\left((\phi-w) \frac{\partial \rho}{\partial t}, \phi-w\right) \mathrm{d} t \\
+\frac{1}{2} \int_{0}^{T}\left((\phi-w) \frac{\partial \rho}{\partial t}, \phi-w\right) \mathrm{d} t+\frac{1}{2} \int_{0}^{T}\left((\phi-w) \frac{\partial \rho}{\partial t}, \phi-w\right) \mathrm{d} t \\
=\frac{1}{2} \frac{d}{\mathrm{~d} t} \int_{0}^{T}\left\|\rho^{1 / 2}(\phi-w)\right\|^{2} \mathrm{~d} t+\frac{1}{2} \int_{0}^{T}\left((\phi-w) \frac{\partial \rho}{\partial t}, \phi-w\right) \mathrm{d} t
\end{gathered}
$$

Usando a equação da densidade, obtemos as relações seguintes:

(i) $\int_{0}^{T}\left(\frac{\partial \rho}{\partial t} \phi, \phi-w\right) \mathrm{d} t=-\int_{0}^{T}(\operatorname{div}(\rho u) \phi, \phi-w) \mathrm{d} t$

(ii) $\int_{0}^{T}\left((\phi-w) \frac{\partial \rho}{\partial t}, \phi-w\right) \mathrm{d} t=-\int_{0}^{T} \int_{r} \operatorname{div}(\rho u)(\phi-w) \cdot(\phi-w) \mathrm{d} x$ 
Substituindo (i), (ii), junto com (4.17) em (4.16), obtém-se

$$
\begin{aligned}
& \int_{0}^{T}\left(\rho \frac{\partial \phi}{\partial t}, \phi-w\right)-(\operatorname{div}(\rho u) \phi, \phi-w)-(\rho w, u \cdot \nabla(\phi-w))+B(w, \phi-w) \\
& +4 \mu_{r}(w, \phi-w)-2 \mu_{r}(\operatorname{rot}(u), \phi-w)+\frac{1}{2}(\operatorname{div}(\rho u)(\phi-w), \phi-w) \\
\geq & \int_{0}^{T}(\rho g, \phi-w) \mathrm{d} t+\frac{1}{2} \frac{d}{\mathrm{~d} t} \int_{0}^{T}\left\|\rho^{1 / 2}(\phi-w)\right\|^{2} \mathrm{~d} t
\end{aligned}
$$

Agora, vejamos o termo seguinte,

$$
-(u \cdot \nabla \rho \phi, \phi-w)+\frac{1}{2}(u \cdot \nabla \rho(\phi-w), \phi-w)-(\rho w, u \cdot \nabla(\phi-w)) .
$$

Observemos que

$$
\int_{\Omega}\left(u \cdot \rho(\phi-w) \cdot(\phi-w) \mathrm{d} x=\int_{\Omega}\left(u \cdot \nabla \rho(\phi-w)(\phi-w)+2 \int_{\Omega} \rho u \cdot(\rho-w) \nabla(\phi-w)=0\right.\right.
$$

e portanto

$\int_{\Omega} u \cdot \nabla \rho(\phi-w) \cdot(\phi-w) \mathrm{d} x=-2 \int_{\Omega} \rho \cdot \nabla(\phi-w)(\phi-w)+2 \int_{\Omega} \rho u \cdot(\rho-w) \nabla(\phi-w)=0$

Além disso,

$\int_{\Omega} \nabla(\rho u \phi \cdot(\phi-w))=\int_{\Omega} u \nabla \rho \phi \cdot(\phi-w) \mathrm{d} x+\int_{\Omega} \rho u \cdot \nabla \phi(\phi-w) \mathrm{d} x+\int_{\Omega} \rho u \cdot \nabla(\phi-w) \phi \mathrm{d} x=0$.

Logo,

$$
\int_{\Omega} u \cdot \nabla \rho \phi \cdot(\phi-w) \mathrm{d} x=-\int_{\Omega} \rho u \nabla \phi(\phi-w) \mathrm{d} x-\int_{\Omega} \rho u \phi \cdot \nabla(\phi-w) \mathrm{d} x
$$

Portanto, usando (4.20), (4.21) em (4.19), reescrita na forma integral, tem-se

$$
\begin{aligned}
& \int_{\Omega} \rho u \cdot \nabla \phi(\phi-w) \mathrm{d} x+\int_{\Omega} \rho u \phi \cdot \nabla(\phi-w) \mathrm{d} x-\int_{\Omega} \rho u \cdot \nabla(\phi-w)(\phi-w) \\
& \quad-\int_{\Omega} \rho w u \cdot \nabla(\phi-w) \\
& =\int_{\Omega} \rho u \cdot \nabla \phi(\phi-w)+\int_{\Omega} \rho \phi(u \cdot \nabla) \phi-\int_{\Omega} \rho \phi(u \cdot \nabla) w-\int_{\Omega} \rho(u \cdot \nabla(\phi-w)) \phi \\
& \quad+\int_{\Omega} \rho(u \cdot \nabla)(\phi-w) w-\int_{\Omega} \rho w(u \cdot \nabla)(\phi-w)
\end{aligned}
$$




$$
\begin{aligned}
& =\int_{\Omega} \rho(u \cdot \nabla \phi) \phi-\int_{\Omega} \rho(u \cdot \nabla \phi) w+\int_{\Omega} \rho(u \cdot \nabla \phi) \phi-\int_{\Omega} \rho(u \cdot \nabla w) \phi \mathrm{d} x \\
& \quad-\int_{\Omega} \phi \rho(u \cdot \nabla \phi)+\int_{\Omega} \rho(u \cdot \nabla w) \phi+\int_{\Omega} \rho(u \cdot \nabla \phi) w-\int_{\Omega} \rho(u \cdot \nabla w) w \\
& -\int_{\Omega} \rho(u \cdot \nabla \phi) w+\int_{\Omega} \rho(u \cdot \nabla w) w \\
& =(\rho u \cdot \nabla \phi, \phi-w)
\end{aligned}
$$

Portanto, finalmente

$$
\begin{aligned}
& \int_{0}^{T}\left(\rho \frac{(\partial \phi)}{\partial t}, \phi-w\right)+(\rho u \cdot \nabla \phi, \phi-w)+B(w, \phi-w)+4 \mu_{r}(w, \phi-w) \\
& -2 \mu_{r}(\operatorname{rot} u, \phi-w)-(\rho g, \phi-w) \mathrm{d} t \\
\geq & -\frac{1}{2}\left\|\rho^{1 / 2}(0)(\phi(0)-w(0))\right\|^{2}
\end{aligned}
$$

Portanto, dizemos que as funções $(u, w, \rho)$ é solução fraca do sistema (4.1) - (4.5) e se verificam as relações seguintes:

(a) $\int_{0}^{T}\left(\rho \frac{\partial v}{\partial t}, v-u\right)+(\rho u \cdot \nabla v, v-u)+\mu a(u, v-u)+2 \mu_{r}(\operatorname{rot} w, v-u)$ $\geq(\rho f, v-u)-\frac{1}{2}\left\|\rho^{1 / 2}(0)(v(0)-u(0))\right\|^{2}$ $u \in L^{2}(0, T: V), u \in \mathbb{K}_{1}, \rho \in L^{\infty}(Q), \forall v \in \Psi, v \in \mathbb{K}_{1}$,

(b) $\int_{0}^{T}\left\{\left(\rho \frac{\partial \phi}{\partial t}, \phi-w\right)+(\rho u \cdot \nabla \phi, \phi-w)+B(w, \phi-w)+4 \mu a(w, \phi-w)\right.$ $\left.-2 \mu_{r}(\operatorname{rot}(u), \phi-w)-(\rho g, \phi-w)\right\} \mathrm{d} t \geq-\frac{1}{2}\left\|\rho^{1 / 2}(0)(\phi(0)-w(0))\right\|^{2}$ $w \in L^{2}(0, T ; H(\Omega)), w \in \mathbb{K}_{2}$,

(c) $\frac{\partial \rho}{\partial t}+\operatorname{div}(\rho u)=0$

(d) $u(x, 0)=u_{0}(x), w(x, 0)=w_{0}(x), \rho(x, 0)=\rho_{0}(x)$, $u \equiv 0$ sobre $\Sigma_{T}=\partial \Omega \times(0, T)$.

\subsection{Existência de soluções fracas}

Nesta seção, vamos enunciar o resultado principal deste capítulo (Teorema 4.1) e todas as outras seções são usadas para provar este resultado. 
Teorema 4.1 Assumindo as seguintes hipóteses:

$$
\begin{aligned}
& f \in L^{2}(0, T ; H), g \in L^{2}\left(0, T ; L^{2}(\Omega)\right), \\
& u_{0} \in H, w_{0} \in L_{2}(\Omega), u_{0} \in K_{1}, \\
& \rho_{0} \in L^{\infty}\left(Q_{T}\right), 0<m \leq \rho_{0}(x) \leq M .
\end{aligned}
$$

Então existem distribuições $(u, w, \rho)$ satisfazendo

$$
\begin{aligned}
& u \in L^{2}(0, T ; V) ; \quad w \in L^{2}\left(0, T ; H^{1}(\Omega)^{3}\right) \\
& \rho \in L^{\infty}\left(Q_{T}\right) \quad e \quad u \in K_{1}, w \in K_{2} .
\end{aligned}
$$

Satisfazendo as relações (a) - (d) da seçâo anterior para todo $v \in \Psi, v \in \mathbb{K}_{1}$ e para todo $\phi \in \Psi, \phi \in \mathbb{K}_{2}$.

Nesta seção consideremos $P_{k}^{1}$ como o operador projeção de $L_{2}(\Omega)^{3}$ sobre $\mathbb{K}_{1}$, e ponhamos

$$
Q^{1} v=v-P_{K}^{1} v
$$

O operador $Q^{1}$ é monótono e hemi-contínuo valendo as seguintes relações:

$$
\begin{aligned}
\left(v-P_{K}^{1} v, P_{k} v\right) & \geq 0, \\
\left(v-P_{K}^{1}, P_{K} v-h\right) & \geq 0, \quad \forall h \in K_{1} .
\end{aligned}
$$

Analogamente, definimos o operador de projeção $P_{K}^{2}: L^{2}(\Omega)^{3} \mapsto \mathbb{K}_{2}$ e, além disso, $Q^{2} v=v-P_{K}^{2} v$ é monótono e hemi-contínuo satisfazendo as relações (4.22) e (4.23).

\subsubsection{Problema auxiliar}

Vamos considerar famílias de "aproximações internas" $V_{m}$ de $V$ e $W_{m}$ de $H^{1}(\Omega)$. Em relação a estas "famílias de aproximações internas"faremos as seguintes hipóteses. 
(i) $V_{m}, W_{m}$ são subespaços de $V$ e $H^{1}(\Omega)$ de dimensão finita $m$;

(ii) $\forall v \in V$, existem sequências $v_{m} \in V_{m}, w_{m} \in W_{m}, w \in H^{1}(\Omega)$ tais que $v_{m} \rightarrow v$ e $w_{m} \rightarrow w$

(iii) Todas as componentes das funções $v$ (respectivamente de $u$ ) em $V_{m}$ (respectivamente de $W_{m}$ ) pertencem a $C^{1}(\bar{\Omega})$.

Já que $V$ é denso em $H$ e $u_{0} \in H$, é possível encontrar $u_{0 m} \in V_{m}$ tal que $u_{0 m} \rightarrow u_{0}$ em $H$ quando $m \rightarrow \infty$. Similarmente como $H^{1}(\Omega)^{3}$ é denso em $L^{2}(\Omega)^{3}$ é possível encontrar $w_{0 m} \in W_{m}$ tal que $w_{0 m} \rightarrow w_{0}$ quando $m \rightarrow \infty$.

Consideremos agora, $\left\{v_{1}, v_{2}, \cdots, v_{m}\right\}$ e $\left\{\phi_{1}, \phi_{2}, \cdots, \phi_{n}\right\}$ bases de $V_{m}$ e $W_{m}$ respectivamente e consideremos o sistema seguinte

$$
\begin{aligned}
& \left(\rho_{m} \frac{\partial u_{m}}{\partial t}, v_{k}\right)+\int_{\Omega} \rho_{m}(u \cdot \nabla) u_{m} v_{k} \mathrm{~d} x+\left(\mu+\mu_{r}\right) a\left(u_{m}, v_{k}\right) \\
& +m\left(Q^{1}\left(u_{m}\right), v_{k}\right)-2 \mu_{r}\left(\operatorname{rot} w_{m}, v_{k}\right)=\left(\rho_{m} f, v_{k}\right) \\
& \left(\rho_{m} \frac{\partial w_{m}}{\partial t}, \phi_{k}\right)+\int_{\Omega} \rho_{m}(u \cdot \nabla) w_{m} \phi_{k} \mathrm{~d} x+B\left(w_{m}, \phi_{k}\right)+m\left(Q^{2}\left(w_{m}\right), \phi_{k}\right) \\
& -2 \mu_{r}\left(\operatorname{rot}\left(u_{m}\right)\right)+4 \mu_{r}\left(w_{m}, \phi_{k}\right)=\left(\rho_{m} g, \phi_{k}\right) \\
& \frac{\partial \rho_{m}}{\partial t}+u_{m} \cdot \nabla \rho_{m}=0 \\
& u_{m}(0)=u_{0 m} \in V_{m}, u_{0 m} \rightarrow u_{0} \mathrm{em} H \\
& w_{m}(0)=u_{0 m} \in W_{m}, w_{0 m} \rightarrow w_{0} \mathrm{em} L_{2}(\Omega)^{3} \\
& \rho_{m}(0)=\rho_{0 m}, \quad \rho_{0 m} \in C^{1}(\bar{\Omega}) \\
& \frac{1}{m} \leq \rho_{0 m} \leq \beta \quad \rho_{0 m} \rightarrow \rho_{0} \text { em } L^{\infty}(\Omega)
\end{aligned}
$$


Ponhamos agora

$$
u_{m}=\sum_{k=1}^{m} C_{k m}(t) v_{k} \text { e } w_{m}=\sum_{k=1}^{m} d_{k m}(t) \phi_{k}
$$

Logo, multiplicando (4.24) por $c_{k m}$ e (4.25) por $d_{k m}(t)$, depois de somar de 1 até $k$ obtém-se

$$
\begin{aligned}
& \left(\rho^{m} \frac{\partial u^{m}}{\partial t}, u^{m}\right)+\left(\rho^{m}\left(u^{m} \cdot \nabla\right) u^{m}, u^{m}\right)+a\left(u^{m}, u^{m}\right) \\
& +m\left(Q^{1}\left(u^{m}\right), u^{m}\right)-2 \mu_{r}\left(\operatorname{rot} w^{m}, u^{m}\right)=\left(\rho^{m} f, u^{m}\right)
\end{aligned}
$$

e

$$
\left(\rho^{m} \frac{\partial w^{m}}{\partial t}, w^{m}\right)+\left(\rho^{m}\left(u^{m} \cdot \nabla\right) w^{m}, w^{m}\right)+B\left(w^{m}, w^{m}\right)+4 \mu_{r}\left(\operatorname{rot} u^{m}, w^{m}\right)=\left(\rho^{m} g, w^{m}\right)
$$

Além disso, temos as relações

$$
\begin{aligned}
& \left(\rho^{m} \frac{\partial u^{m}}{\partial t}, u^{m}\right)=\frac{\rho^{m}}{2} \frac{\partial}{\partial t}\left(u^{m}\right)^{2}, \\
& \left(\rho^{m} \frac{\partial w^{m}}{\partial t}, w^{m}\right)=\frac{\rho^{m}}{2} \frac{\partial}{\partial t}\left(w^{m}\right)^{2}, \\
& \frac{1}{2} \int_{\Omega} \rho^{m} u^{m} \cdot \nabla\left(u^{m}\right)^{2}=\int_{\Omega} \rho^{m}\left(u^{m} \cdot \nabla u^{m}\right) u^{m}, \\
& \frac{1}{2} \int_{\Omega} \rho^{m}\left(u^{m} \cdot \nabla\right)\left(w^{m}\right)^{2}=\int_{\Omega} \rho^{m}\left(u^{m} \cdot \nabla w^{m}\right) w^{m} .
\end{aligned}
$$

Logo, usando estas últimas expressões, vem que

$$
\begin{aligned}
& \frac{1}{2} \int_{\Omega} \rho^{m} \frac{\partial}{\partial t}\left(u^{m}\right)^{2}+\frac{1}{2} \int_{\Omega} \rho^{m}\left(u^{m} \cdot \nabla\right)\left(u^{m}\right)^{2}+a\left(u^{m}, u^{m}\right)+m\left(Q^{1}\left(u^{m}\right), u^{m}\right) \\
& -2 \mu_{r}\left(\operatorname{rot} w^{m}, u^{m}\right)=\left(\rho^{m} f, w^{m}\right)
\end{aligned}
$$

e

$$
\begin{aligned}
& \frac{1}{2} \int_{\Omega} \rho^{m} \frac{\partial}{\partial t}\left(w^{m}\right)^{2}+\frac{1}{2} \int_{\Omega} \rho^{m}\left(u^{m} \cdot \nabla\right)\left(w^{m}\right)^{2}+B\left(w^{m}, w^{m}\right)+4 \mu_{r}\left(w^{m}, w^{m}\right) \\
& +m\left(Q^{2}\left(w^{m}\right), w^{m}\right)-2 \mu_{r}\left(\operatorname{rot} u^{m}, w^{m}\right)=\left(\rho^{m} g, w^{m}\right)
\end{aligned}
$$

Multiplicando a equação da densidade (4.26) por $\frac{\left(u^{m}\right)^{2}}{2}$ e integrando sobre $\Omega$, obtém-se

$$
\int_{\Omega} \frac{\partial \rho^{m}}{\partial t} \frac{\left(u^{m}\right)^{2}}{2} \mathrm{~d} x+\int_{\Omega} u^{m} \cdot \nabla \rho^{m} \frac{\left(u^{m}\right)^{2}}{2} \mathrm{~d} x=0
$$


e somando essa última quantidade a (4.28) obtém-se

$$
\begin{aligned}
& \frac{\partial}{\partial t} \int_{\Omega} \rho^{m} \frac{\left(u^{m}\right)^{2}}{2}+\int_{\Omega} \operatorname{div}\left(\rho^{m} u^{m} \frac{\left(u^{m}\right)^{2}}{2}\right) \mathrm{d} x+\left(\mu+\mu_{r}\right) a\left(u^{m}, u^{m}\right) \\
& +m\left(u^{m}-P_{k}^{1} u^{m}, u^{m}-P_{k}^{1} u^{m}\right)+m\left(u^{m}-P_{k}^{1} u^{m}, P_{k}^{1} u^{m}\right) \\
& -2 \mu_{r}\left(\operatorname{rot} w^{m}, u^{m}\right)=\left(\rho^{m} f, u^{m}\right)
\end{aligned}
$$

Similarmente, multiplicando (4.26) por $w^{m}$, obtemos

$$
\int_{\Omega} \frac{\partial \rho^{m}}{\partial t} \frac{\left(w^{m}\right)^{2}}{2}+\int_{\Omega}\left(u^{m} \cdot \nabla\right) \rho^{m} \frac{\left(w^{m}\right)^{2}}{2} \mathrm{~d} x=0
$$

Somando essa quantidade a (4.29), obtém-se

$$
\begin{aligned}
& \int_{\Omega} \frac{\partial}{\partial t} \rho^{m} \frac{\left(w^{m}\right)^{2}}{2}+\int_{\Omega} \rho^{m} \cdot \nabla \rho^{m} \frac{\left(w^{m}\right)^{2}}{2}+\int_{\Omega} \rho^{m}\left(u^{m} \cdot \nabla\right) \frac{\left(w^{m}\right)^{2}}{2}+B\left(w^{m}, w^{m}\right) \\
& +m\left\|w^{m}-P_{k}^{2} w^{m}\right\|^{2}+m\left(w^{m}-P_{k}^{2} w^{m}, P_{k}^{2} w^{m}\right)+4 \mu_{r}\left(w^{m}, w^{m}\right)-2 \mu_{r}\left(\operatorname{rot} u^{m}, w^{m}\right) \\
& =\left(\rho^{m} g, w^{m}\right)
\end{aligned}
$$

Observe-se (usando coordenadas) que

$$
\frac{\partial}{\partial x_{j}}\left(u_{m_{j}} \rho_{m} \frac{w_{m_{i}}^{2}}{2}\right)=\frac{\partial u_{m_{j}}}{\partial x_{j}} \rho_{m} \frac{w_{m_{i}}^{2}}{2}+u_{m_{j}} \frac{\partial \rho}{\partial x_{j}} \frac{w_{m_{i}}^{2}}{2}+u_{m_{j}} \rho_{m} \frac{\partial}{\partial x_{j}}\left(\frac{w_{m_{i}}^{2}}{2}\right)
$$

Usando esta expressão e levando em conta o teorema da divergência, obtemos

$$
\int_{\Omega}\left(u_{m} \cdot \rho_{m}\right) \frac{\left(w_{m}\right)^{2}}{2} \mathrm{~d} x+\int_{\Omega} u_{m} \rho_{m} \cdot \nabla\left(\frac{w_{m}^{2}}{2}\right) \mathrm{d} x=0 .
$$

Finalmente, obtém-se

$$
\begin{aligned}
& \int_{\Omega} \frac{\partial}{\partial t}\left(\rho^{m} \frac{\left(w^{m}\right)^{2}}{2}\right)+B\left(w^{m}, w^{m}\right)+m\left\|w^{m}-P_{k}^{2} w^{m}\right\|^{2}+m\left(w^{m}-P_{k}^{2} w^{m}, P_{k}^{2} w^{m}\right) \\
& +4 \mu_{r}\left(w^{m}, w^{m}\right)=\left(\rho^{m} g, w^{m}\right)
\end{aligned}
$$

Agora somando (4.30) e (4.31), obtém-se

$$
\begin{aligned}
& \frac{1}{2} \frac{\partial}{\partial t}\left(\int_{\Omega} \rho^{m}\left(u^{m}\right)^{2}+\int_{\Omega} \rho^{m}\left(w^{m}\right)^{2}\right)+\left(\mu+\mu_{r}\right)\left\|\nabla u^{m}\right\|^{2}+\mu_{C}\left\|\nabla w^{m}\right\|^{2}+\gamma\left\|\operatorname{div}\left(w^{m}\right)\right\|^{2} \\
& -2 \mu_{r}\left(\operatorname{rot} w^{m}, u^{m}\right)-2 \mu_{r}\left(\operatorname{rot} u^{m}, w^{m}\right)+m\left(\left\|u^{m}-P_{k}^{1} u^{m}\right\|^{2}+\left\|w^{m}-P_{k}^{2} w^{m}\right\|^{2}\right) \\
& +m\left(\left(u^{m}-P_{k}^{1} u^{m}, P_{k}^{1} u^{m}\right)+\left(w^{m}-P_{k}^{2} w^{m}, P_{k}^{2} w^{m}\right)\right)+4 \mu_{r}\left(w^{m}, w^{m}\right) \\
& =\left(\rho^{m} f, u^{m}\right)+\left(\rho^{m} g, w^{m}\right) .
\end{aligned}
$$


Integrando essa expressão com relação a $t$, obtém-se

$$
\begin{aligned}
& \frac{1}{2}\left(\left\|\left(\rho^{m}\right)^{1 / 2} u^{m}\right\|^{2}+\left\|\left(\rho^{m}\right)^{1 / 2} w^{m}\right\|^{2}\right)+\left(\mu+\mu_{r}\right) \int_{0}^{T}\left\|\nabla u^{m}\right\|^{2} \mathrm{~d} s+\nu \int_{0}^{T}\left\|\nabla w^{m}\right\| \mathrm{d} s \\
& +\delta \int_{0}^{T}\left\|\operatorname{div} w^{m}\right\|^{2} \mathrm{~d} s-2 \mu_{r}\left(\int_{0}^{T}\left(\operatorname{rot} w^{m}, u^{m}\right) \mathrm{d} s+\int_{0}^{T}\left(\operatorname{rot} u^{m}, w^{m}\right) \mathrm{d} s\right) \\
& +m\left(\int_{0}^{T}\left(\left\|u^{m}-P_{k}^{1} w^{m}\right\|^{2}+\left\|w^{m}-P_{k}^{2} w^{m}\right\|^{2}\right) \mathrm{d} s+m\left(\int _ { 0 } ^ { T } \left\{\left(u^{m}-P_{k}^{1} u^{m}, P_{k}^{1} u^{m}\right)\right.\right.\right. \\
& \left.+\left(w^{m}-P_{k}^{2} w^{m}, P_{k}^{2} w^{m}\right)\right\} \mathrm{d} s+4 \mu_{r} \int_{0}^{T}\left\|w^{m}\right\|^{2} \mathrm{~d} s \\
& =\int_{0}^{T}\left\{\left(\rho^{m} f, u^{m}\right)+\frac{\rho_{0 m}}{2}\left(\left\|u_{0 m}\right\|^{2}+\left\|w_{0 m}\right\|^{2}\right)\right.
\end{aligned}
$$

ou ainda,

$$
\begin{aligned}
& \left(\left\|\left(\rho^{m}\right)^{1 / 2} u^{m}\right\|^{2}+\left\|\left(\rho^{m}\right)^{1 / 2} w^{m}\right\|^{2}\right)+2 \mu \int_{0}^{T}\left\|\nabla u^{m}\right\|^{2} \mathrm{~d} s++2 \nu \int_{0}^{T}\left\|\nabla w^{m}(s)\right\|^{2} \mathrm{~d} s \\
& +2 \delta \int_{0}^{T}\left\|\operatorname{div}\left(w^{m}\right)\right\|^{2}-4 \mu_{r}\left(\int_{0}^{T}\left\{\left(\operatorname{rot} w^{m}, u^{m}\right)+\left(\operatorname{rot} u^{m}, w^{m}\right)\right\} \mathrm{d} s\right) \\
& +2 m\left(\int_{0}^{T}\left(\left\|u^{m}-P_{k}^{1} u^{m}\right\|^{2}+\left\|w^{m}-P_{k}^{2} w^{m}\right\|^{2}\right) \mathrm{d} s+2 m\left(\int _ { 0 } ^ { T } \left\{\left(u^{m}-P_{k}^{1} u^{m}, P_{k}^{1} u^{m}\right)\right.\right.\right. \\
& \left.+\left(w^{m}-P_{k}^{2} w^{m}, P_{k}^{2} w^{m}\right)\right\} \mathrm{d} s++8 \mu_{r} \int_{0}^{T}\left\|w^{m}\right\|^{2} \mathrm{~d} s \\
& =\frac{1}{2} \rho_{0 m}\left(\left\|u_{0 m}\right\|^{2}+\left\|w_{0 m}\right\|^{2}\right)++2 \int_{0}^{T}\left\{\left(\rho^{m} f, u^{m}\right)+\left(\rho^{m} g, w^{m}\right)\right\} \mathrm{d} s
\end{aligned}
$$

\subsubsection{Estimativas a priori}

Agora vamos fazer algumas limitações sobre alguns termos da igualdade (4.32).

$$
\begin{aligned}
& 2\left(\rho^{m} f, u^{m}\right) \leq 2\left\|\rho^{m}\right\|_{L^{\infty}}\|f\|_{L^{2}}\left\|u^{m}\right\|_{L^{2}} \leq C_{\epsilon}\|f\|^{2}+\epsilon\left\|\nabla u^{m}\right\|^{2} \\
& 2\left(\rho^{m} g, u^{m}\right) \leq C_{\delta}\|g\|^{2}+\delta\left\|\nabla w^{m}\right\|^{2} \\
& 4 \mu_{r}\left(\operatorname{rot} u^{m}, w^{m}\right) \leq 4 \mu_{r}\left\|w^{m}\right\|\left\|\operatorname{rot} u^{m}\right\| \leq 4 \mu_{r}\left\|w^{m}\right\|\left\|\nabla u^{m}\right\| \\
& \leq 4 \mu_{r}\left\|w^{m}\right\|^{2}+\mu_{r}\left\|\nabla u^{m}\right\|^{2} \\
& \left|4 \mu_{r}\left(\operatorname{rot} w^{m}, u^{m}\right)\right| \leq 4 \mu_{r}\left\|u^{m}\right\|\left\|\operatorname{rot} w^{m}\right\| \leq 4 \mu_{r}\left\|u^{m}\right\|\left\|\nabla w^{m}\right\| \\
& \leq 4 \mu_{r}\left\|u^{m}\right\|^{2}+\mu_{r}\left\|\nabla w^{m}\right\|^{2}
\end{aligned}
$$

Usando as desigualdades (4.33) - (4.36), obtém-se

$$
\left(\left\|\left(\rho^{m}\right)^{1 / 2} u^{m}\right\|^{2}+\left\|\rho^{m} w^{m}\right\|^{2}\right)+2 \mu \int_{0}^{T}\left\|\nabla u^{m}\right\|^{2} \mathrm{~d} s+C_{1} \int_{0}^{T}\left\|\nabla w^{m}\right\|^{2} \mathrm{~d} s
$$




$$
\begin{aligned}
& +C_{2} \int_{0}^{T}\left\|\operatorname{div} w^{m}\right\|^{2} \mathrm{~d} s+2 m\left(\int _ { 0 } ^ { T } \left(\left\|u^{m}-P_{k}^{1} u^{m}\right\|^{2} \mathrm{~d} s+\int_{0}^{T}\left(\left\|w^{m}-P_{k}^{2} w^{m}\right\|^{2} \mathrm{~d} s\right.\right.\right. \\
& +2 m\left(\int_{0}^{T}\left\{\left(u^{m}-P_{k}^{1} u^{m}, P_{k}^{1} u^{m}\right)+\left(w^{m}-P_{k}^{2} w^{m}, P_{k}^{2} w^{m}\right)\right\} \mathrm{d} s\right. \\
& \leq C \int_{0}^{T}\left(\|g\|_{L^{2}}^{2}+\|f\|_{L^{2}}^{2}\right) \mathrm{d} s++\frac{\rho_{0 m}}{2}\left(\left\|u_{0 m}\right\|^{2}+\left\|w_{0 m}\right\|^{2}\right) .
\end{aligned}
$$

Desta estimativa deduzimos que

$$
\begin{aligned}
& \left(\rho^{m}\right)^{1 / 2} u^{m} \text { está limitada em } L^{\infty}(0, T ; H) \\
& \left(\rho^{m}\right)^{1 / 2} w^{m} \text { está limitada em } L^{\infty}\left(0, T ; L_{2}(\Omega)^{3}\right) ; \\
& u^{m} \text { está limitada em } L^{2}(0, T ; V) \cap L^{\infty}(0, T ; H) ; \\
& w^{m} \text { está limitada em } L^{2}\left(0, T ; H_{0}^{1}(\Omega)\right) \cap L^{\infty}\left(0, T ; L^{2}(\Omega)^{3}\right) .
\end{aligned}
$$

Devido à imersão de Sobolev $H_{0}^{1}(\Omega)^{3} \hookrightarrow L^{6}(\Omega)^{3}$, obtém-se

$$
u^{m}, w^{m} \text { estão limitadas em } L^{2}\left(0, T ; L^{6}(\Omega)^{3}\right)
$$

Usando o fato que $\left\{w^{m}\right\}$ está limitada em $L^{\infty}\left(0, T ; L^{2}(\Omega)^{3}\right)$ junto com (4.41), obtém-se

$$
\begin{aligned}
\left\|w^{m}\right\|_{L^{4}}^{8 / 3} & \left.=\int_{\Omega}\left|w^{m} \| w^{m}\right|^{3}\right)^{2 / 3} \leq\left(\left\|w^{m}\right\|_{L^{2}(\Omega)^{3}}\left\|w^{m}\right\|_{L^{6}(\Omega)^{3}}^{2}\right)^{2 / 3} \\
& =\left\|w^{m}\right\|_{L^{2}(\Omega)^{3}}^{2 / 3}\left\|w^{m}\right\|_{L^{6}(\Omega)^{3}}^{3}
\end{aligned}
$$

Como $\left\|w^{m}\right\|_{L^{2}(\Omega)^{3}}^{2 / 3}$ está limitada no $L^{\infty}(0, T)$ e $\left\|w^{m}\right\|$ limitada em $L^{1}(0, T)$ obtemos que $\left\|w^{m}\right\|_{L^{4}(\Omega)^{3}}^{8 / 3}$ está limitada no $L^{1}(0, T)$. Logo

$$
\begin{aligned}
& u^{m}, w^{m} \text { estão limitadas em } L^{8 / 3}\left(0, T ; L^{4}(\Omega)^{3}\right), \\
& m \int_{0}^{T}\left\|u^{m}-P_{k}^{1} u^{m}\right\|^{2} \mathrm{~d} s \leq C_{1}, \\
& m \int_{0}^{T}\left\|w^{m}-P_{k}^{2} w^{m}\right\|^{2} \mathrm{~d} s \leq C_{2} .
\end{aligned}
$$

Observe-se ainda que

$$
\left\|u^{m} \otimes u^{m}\right\|_{L^{3 / 2}(\Omega)}^{2} \leq\left\|u^{m}\right\|_{L^{4}(\Omega)^{3}}^{4}
$$


Como $u^{m} \in L^{2}(0, T ; V) \cap L^{\infty}(0, T ; H) \subseteq L^{4}\left(0, T ; L^{4}(\Omega)^{3}\right)$, temos

$$
\rho^{m} u^{m} \otimes u^{m} \text { está limitada em } L^{2}\left(0, T ; L^{3 / 2}(\Omega)^{9}\right) .
$$

Agora,

$$
\begin{aligned}
\int_{0}^{T}\left\|u^{m} \otimes w^{m}\right\|_{L^{3 / 2}(\Omega)^{9}}^{2} & \leq \int_{0}^{T}\left\|u^{m}\right\|_{L^{3}(\Omega)^{3}}^{2}\left\|w^{m}\right\|_{L^{3}(\Omega)^{3}}^{2} \\
& \leq\left(\int_{0}^{T}\left\|u^{m}\right\|_{L^{3 / 2}(\Omega)^{3}}^{4}\right)^{1 / 2}\left(\int_{0}^{T}\left\|w^{m}\right\|_{L^{3 / 2}(\Omega)^{3}}^{4}\right)^{1 / 2}
\end{aligned}
$$

Portanto,

$$
\left(\int_{0}^{T}\left\|u^{m} \otimes w^{m}\right\|_{L^{3 / 2}(\Omega)}^{2} \mathrm{~d} s\right)^{1 / 2} \leq\left(\int_{0}^{T}\left\|u^{m}\right\|_{L^{3}(\Omega)^{3}}^{4}\right)^{1 / 4}\left(\int_{0}^{T}\left\|w^{m}\right\|_{L^{3}(\Omega)^{3}}^{4}\right)^{1 / 4} .
$$

Como $u^{m}, w^{m}$ estão limitadas em $L^{\alpha}\left(0, T ; L^{\beta}(\Omega)^{3}\right)$ se $\frac{\beta p}{6}+\frac{\beta(1-p)}{2}=1 \mathrm{com}$ $p \alpha=2,0<p<1$ queremos $\alpha=4$, disto $p=1 / 2$, além disto $\frac{\beta}{12}+\frac{\beta}{4}=1$, temos $\beta=3$, portanto $u^{m}$ e $w^{m}$ estão limitadas em $L^{4}\left(0, T ; L^{3}(\Omega)^{3}\right)$, o que nos fornece

$$
\rho^{m} u^{m} \oplus w^{m} \text { limitadas em } L^{2}\left(0, T ; L^{3 / 2}(\Omega)^{9}\right)
$$

Disto podemos obter

$$
\begin{aligned}
& \left(\rho^{m}\right)^{1 / 2} u^{m} \rightarrow \alpha_{1} \text { na topologia fraca* de } L^{\infty}(0, T ; H), \\
& \left(\rho^{m}\right)^{1 / 2} w^{m} \rightarrow \alpha_{2} \text { na topologia fraca* de } L^{\infty}\left(0, T ; L^{2}(\Omega)^{3}\right), \\
& \left(u^{m}\right) \rightarrow u \text { na topologia fraca de } L^{2}(0, T ; V), \\
& \left(w^{m}\right) \rightarrow w \text { na topologia fraca* de } L^{2}\left(0, T ; H_{0}^{1}(\Omega)^{3}\right), \\
& \rho^{m} u^{m} \rightarrow \delta_{1} \text { na topologia fraca* de } L^{\infty}(0, T ; H), \\
& \rho^{m} w^{m} \rightarrow \delta_{2} \text { na topologia fraca* de } L^{\infty}\left(0, T ; L^{2}(\Omega)^{3}\right) .
\end{aligned}
$$

Das relações (4.45) e (4.46), obtém-se

$$
\begin{gathered}
\rho^{m} u^{m} \otimes u^{m} \rightarrow \eta_{1} \quad \text { e } \quad \rho^{m} u^{m} \otimes w^{m} \rightarrow \eta_{2} \text { na topologia fraca de } \\
L^{2}\left(0, T ; L^{3 / 2}(\Omega)^{3}\right) .
\end{gathered}
$$


Observe ainda que de (4.43) e (4.44), temos as convergências

$$
\begin{aligned}
& \left(u^{m}-P_{k}^{1} u^{m}\right) \rightarrow 0 \text { na topologia forte de } L^{2}\left(Q_{T}\right)^{3}, \\
& \left(w^{m}-P_{k}^{2} w^{m}\right) \rightarrow 0 \text { na topologia forte de } L^{2}\left(Q_{T}\right)^{3} .
\end{aligned}
$$

Portanto, concluímos que $u-P_{k}^{1} u=0, w-P_{k}^{2} w=0$ e daqui $u \in \mathbb{K}_{1} w \in \mathbb{K}_{2}$.

Falta mostrar agora que

$$
\delta_{1}=\rho_{u}, \quad \delta_{2}=\rho_{w}, \quad \eta_{1}=\rho u \otimes u, \quad \eta_{2}=\rho w \otimes w .
$$

Como

$$
\frac{\partial \rho^{m}}{\partial t}=-\nabla \cdot\left(\rho^{m} u^{m}\right) \text { em } Q_{T}
$$

portanto

$$
<\frac{\partial \rho^{m}}{\partial t}, \varphi>_{H^{-1}(\Omega) H_{0}^{1}(\Omega)}=\left(\rho^{m}, u^{m}, \nabla \varphi\right)_{L^{2}(\Omega) L^{2}(\Omega)}
$$

e assim temos

$$
\left|<\frac{\partial \rho^{m}}{\partial t}, \varphi>\right|_{H^{-1} H_{0}^{1}} \leq \|\left(\rho^{m}, u^{m}\left\|_{L}^{2}\right\| \nabla \varphi \|_{L^{2}(\Omega)}\right.
$$

Tomando $\|\nabla \varphi\| \leq 1$ e como $\left(\rho^{m} u^{m}\right)$ limitada em $L^{\infty}\left(0, T ; L^{2}(\Omega)^{3}\right)$ obtém-se que $\frac{\partial \rho^{m}}{\partial t}$ está limitada em $L^{2}\left(0, T ; H^{-1}(\Omega)\right)$, logo

$\left.\begin{array}{l}\rho^{m} \in L^{\infty}\left(0, T ; L^{\infty}(\Omega)\right) \\ \frac{\partial \rho^{m}}{\partial t} \text { em } L^{2}\left(0, T ; H^{-1}(\Omega)\right) \\ L^{\infty}(\Omega) \rightarrow H^{-1} \text { compacto }\end{array}\right\} \Longrightarrow \begin{aligned} & \rho^{m} \rightarrow \rho \text { na topologia forte de } L^{2}\left(0, T ; H^{-1}(\Omega)\right) \\ & \text { (na verdade em } C\left([0, T] ; H^{-1}(\Omega)\right),\end{aligned}$ ou seja

$$
\rho^{m} \rightarrow \rho \text { na topologia fraca de } L^{2}\left(0, T ; H^{-1}(\Omega)\right)
$$

Como podemos escrever

$$
\int_{0}^{T}\left(\rho^{m} u^{m}-\rho u, \varphi\right)_{H^{-1} H_{0}^{1}} \mathrm{~d} t=\int_{0}^{T}\left(u^{m}\left(\rho^{m}-\rho\right), \varphi\right)_{H^{-1} H_{0}^{1}} \mathrm{~d} t+\int_{0}^{T}\left(\rho\left(u^{m}-u\right), \varphi\right)_{H^{-1} H_{0}^{1}} \mathrm{~d} t
$$


tomando em $\varphi \in D$, obtemos

$$
\begin{aligned}
\mid \int_{0}^{T}\left(u^{m}\left(\rho^{m}-\rho\right), \varphi \mathrm{d} t \mid\right. & \leq \sup _{\operatorname{supp}(\varphi)}|\varphi| \int_{0}^{T}\left\|u^{m}\right\|\left\|\rho^{m}-\rho\right\|_{H^{-1}(\Omega)} \mathrm{d} t \\
& \leq \sup _{\operatorname{supp}(\varphi)}|\varphi|\left(\int_{0}^{T}\left\|u^{m}\right\|^{2} \mathrm{~d} s\right)^{1 / 2}\left(\int_{0}^{T}\left\|\rho^{m}-\rho\right\|_{H^{-1}}^{2} \mathrm{~d} s\right)^{1 / 2} .
\end{aligned}
$$

Devido a (4.55), a expressão do lado direito converge para zero quando $m \rightarrow \infty$. Similarmente,

$$
\mid\left(\int_{0}^{T}\left(\rho^{m}\left(u^{m}-u\right), \varphi\right)_{H^{-1} H_{0}^{1}} \mathrm{~d} s|=| \int_{0}^{T}<\left(u^{m}-u\right), \rho \varphi>\mathrm{d} s \mid\right.
$$

converge para zero quando $m$ tende para $+\infty$ devido a (4.49). Portanto $\rho^{n} u^{m} \rightarrow \rho u$ na topologia fraca de $L^{2}\left(0, T ; H^{-1}(\Omega)^{3}\right)$ e como $L^{\infty}\left(0, T ; H^{3}\right) \subseteq L^{2}\left(0, T ; H^{-1}(\Omega)^{3}\right)$, obtém-se que $\delta_{1}=\rho u$. Usando o mesmo argumento anterior concluímos que $\delta_{2}=\rho w$.

\subsection{Estimativas no tempo e passagem ao limite}

Para mostrar que $n_{1}=\rho u \otimes u, \eta_{2}=\rho u \otimes w$ em (4.54) é necessário usar o resultado de compacidade de J. Simon, Lema 1.5 do Capítulo 1 . No nosso caso, $q=2, X=$ $L^{2}(\Omega)^{3}, B=H^{-1}(\Omega)^{3}, Y=W^{-1, r}(\Omega)^{3}$ com $r<3 / 2$. Vamos obter convergências forte de $\rho^{m} u^{m} \rightarrow \rho u, \rho^{m} w^{m} \rightarrow \rho w$ em $L^{2}\left(0, T ; H^{-1}(\Omega)\right)$ e usando o fato de que $u^{m} \rightarrow u$ fraco em $L^{2}(0, T ; V)$ e $w^{m} \rightarrow w$ fraco em $L^{2}\left(0, T ; H_{0}^{1}(\Omega)\right)$, obtém-se $\eta_{1}=$ $\rho u \otimes u$ e $\eta_{2}=\rho w \otimes u$. Para fazer nosso plano, devemos obter estimativas do tipo

$$
\begin{aligned}
& \left\|\left(\rho^{m} u^{m}\right)_{h}-\rho^{m} u^{m}\right\|_{L^{2}\left(0, T ; W-1, r(\Omega)^{3}\right)} \leq C h^{s}, \\
& \left\|\left(\rho^{m} w^{m}\right)_{h}-\rho^{m} w^{m}\right\|_{L^{2}\left(0, T ; W^{-1, r}(\Omega)\right)} \leq C h^{s},
\end{aligned}
$$

para algum $s$ adequado para que $\left(\rho^{m} u^{m}\right),\left(\rho^{m} w^{m}\right)$ estejam no espaço de Nikolskii $N^{s, 2}\left(0, T ; W^{-1, r}(\Omega)^{3}\right)$. Observe-se que

$$
\tau_{h}\left(\rho^{m} u^{m}\right)-\rho^{m} u^{m}=\tau \rho^{m}\left(\tau_{h} u^{m}-u^{m}\right)+\left(\tau_{h} \rho^{m}-\rho^{m}\right) u^{m},
$$


onde $\tau_{h}$ é a translação definida no Capítulo 1, Seção 1.4. Logo, temos que

$$
\begin{aligned}
\left\|\tau_{h}\left(\rho^{m} u^{m}\right)-\rho^{m} u^{m}\right\|_{L^{2}\left(0, T-h ; W-1, r(\Omega)^{3}\right)} \leq & \left.\left\|\tau_{h} \rho^{m}\left(\tau_{h} u^{m}-u^{m}\right)\right\|_{L^{2}(0, T ; W-1, r}(\Omega)^{3}\right) \\
& +\left\|\left(\tau_{h} \rho^{m}-\rho^{m}\right) u^{m}\right\|_{L^{2}\left(0, T ; W^{-1, r}(\Omega)^{3}\right)}(4.57)
\end{aligned}
$$

com $r<3 / 2$

Devemos notar que os produtos de $\rho^{m} u^{m}$ e $\rho^{m} w^{m}$ fazem sentido em $W^{-1, r}(\Omega)^{3}$ com $r<3 / 2$ devido a que aplicação produto

$$
H_{0}^{-1}(\Omega) \times H_{0}^{1}(\Omega) \rightarrow W^{-1, r}(\Omega) \operatorname{com} r<3 / 2
$$

é contínua pelo Lema 1.4 do Capítulo 1 . Notemos que

$$
\left(\tau_{n} \rho^{m}-\rho^{m}\right)(t)=\rho^{m}(t+h)-\rho^{m}(t)=\int_{t}^{t+h} \frac{\partial \rho^{m}}{\partial t}(s) \mathrm{d} s=-\nabla \cdot \int_{t}^{t+h} \rho^{m}(s) u^{m}(s) \mathrm{d} s
$$

Portanto aplicando a desigualdade de Hölder, obtém-se

$$
\begin{aligned}
\left.\| \rho^{m}(t+h)-\rho^{m}\right)(t) \|_{H^{-1}(\Omega)} & =\sup _{\|\nabla \varphi\| \leq 1}<-\nabla \cdot \int_{t}^{t+h}\left(\rho^{m} u^{m}\right)(s) \mathrm{d} s, \varphi> \\
& =\sup _{\|\nabla \varphi\| \leq 1}\left(\int_{t}^{t+h}\left(\rho^{m} u^{m}\right)(s) \mathrm{d} s, \nabla \varphi\right)_{L^{2}} L^{2} \\
& \leq C(h)^{1 / 2}\left(\int_{t}^{t}\left\|\left(u^{m} \rho^{m}\right)(s)\right\|_{L^{2}}^{2} \mathrm{~d} s\right)^{1 / 2}, \quad \forall 0 \leq t \leq T-h .
\end{aligned}
$$

Assim,

$$
\left\|\tau_{h} \rho^{m}-\rho^{m}\right\|_{L^{\infty}\left(0, T ; H^{-1}(\Omega)\right)} \leq C h^{1 / 2}
$$

e de fato obtemos

$$
\left\|\tau_{h} \rho^{m}-\rho^{m}\right\|_{L^{2}\left(0, T ; H^{-1}(\Omega)\right)} \leq C h^{1 / 4} .
$$

Como temos imersão contínua de $H^{-1}(\Omega)^{3} \rightarrow\left(W^{-1, r}(\Omega)\right)^{3}$ com $r<3 / 2$ obtém-se

$$
\left\|\tau_{h} \rho^{m}-\rho^{m}\right\|_{L^{2}\left(0, T-h ; W^{-1, r}(\Omega)\right)} \leq C h^{1 / 4}
$$

Agora, vamos fazer $n$ estimativas da forma

$$
\int_{0}^{T-h}\left(\int_{\Omega} \mid \rho^{m}(t+h)\left(u^{m}(t+h)-u^{m}(t)\right)^{2}\right) \mathrm{d} t \leq C h^{1 / 2}
$$


ou escrito de outra forma.

$$
\left\|\tau_{h} \rho^{m}\left(\tau_{h} u^{m}-u^{m}\right)\right\|_{L^{2}\left(0, T ; L^{2}(\Omega)^{3}\right)} \leq C h^{1 / 4}
$$

e

$$
\left\|\tau_{n} \rho^{m}\left(\tau_{n} w^{m}-w^{m}\right)\right\|_{L^{2}\left(0, T ; L^{2}(\Omega)^{3}\right)} \leq C h^{1 / 4}
$$

Levando em conta a imersão contínua de $L^{2}(\Omega) \hookrightarrow W^{-1, r}(\Omega)$, obtemos

$$
\|\left(\tau_{h} \rho^{m}\left(\tau_{h} u^{m}-u^{m}\right) \|_{L^{2}\left(0, T-h ; W^{-1, r}(\Omega)^{3}\right)} \leq C h^{1 / 4}\right.
$$

e ainda tém-se

$$
\|\left(\tau_{h} \rho^{m}\left(\tau_{h} w^{m}-w^{m}\right) \|_{L^{2}\left(0, T-h ; W^{-1,3}(\Omega)^{3}\right)} \leq C h^{1 / 4}\right.
$$

Usando (4.60), (4.63), (4.40) em (4.57), obtém-se

$$
\begin{aligned}
\|\left(\tau_{h}\left(\rho^{m} u^{m}\right)-\rho^{m} u^{m} \|_{L^{2}\left(0, T-h ; W^{-1, r}(\Omega)^{3}\right)}\right. & \leq C h^{1 / 4} \\
\|\left(\tau_{h}\left(\rho^{m} w^{m}\right)-\rho^{m} w^{m} \|_{L^{2}\left(0, T-h ; W^{-1, r}(\Omega)^{3}\right)}\right. & \leq C h^{1 / 4},
\end{aligned}
$$

resultando que $\left(\rho^{m} u^{m}\right),\left(\rho^{m} w^{m}\right)$ estão no espaço de Nikolskii $N^{1 / 4, s}\left(0, T ; W^{-1, r}(\Omega)^{3}\right)$.

Ponhamos $u_{h m}=\frac{1}{h} \int_{t-h}^{t} u^{m}(s) \mathrm{d} s$ e substituindo $v_{k}$ por $u_{h m}$ na equação (4.29), obtém-se

$$
\begin{aligned}
& \int_{h}^{T}\left\{\left(\rho^{m} \frac{\partial u^{m}}{\partial t}, u_{h m}\right)+\left(\rho^{m}\left(u^{m} \cdot \nabla\right) u^{m}, u_{h m}\right)+\left(\mu+\mu_{r}\right) a\left(u^{m}, u_{h m}\right)\right. \\
& \left.+m\left(Q^{1}\left(u^{m}\right), u_{h m}\right)-2 \mu_{r}\left(\operatorname{rot} w^{m}, u_{h m}\right)\right\} \mathrm{d} t=\int_{h}^{T}\left(\rho^{m} f, u_{h m}\right) \mathrm{d} t
\end{aligned}
$$

Observe-se que por integração por partes obtemos

$$
\begin{aligned}
& \left.\int_{h}^{T}\left(\frac{\partial}{\partial t} \rho^{m} u^{m}\right)(t), \frac{1}{h} \int_{t-h}^{t} u^{m}(s) \mathrm{d} s\right) \mathrm{d} t=\left(\rho^{m}(T) u^{m}(T), \frac{1}{h} \int_{T-h}^{T} u^{m}(s) \mathrm{d} s\right) \\
& \left.-\left(\rho^{m}(h) u^{m}(h), \frac{1}{h} \int_{0}^{h} u^{m}(s) \mathrm{d} s\right)-\frac{1}{h} \int_{h}^{T}\left(\rho^{m} u^{m}\right)(t), u^{m}(t)-u^{m}(t-h)\right) \mathrm{d} t \\
\leq & \left\|\rho^{m}(T) u^{m}(T)\right\|_{L^{2}(\Omega)^{3}}+\frac{1}{h} \int_{T-h}^{T} u^{m}(s) \mathrm{d} s \|_{L^{2}(\Omega)^{3}}
\end{aligned}
$$




$$
\begin{aligned}
& +\left\|\rho^{m}(h) u^{m}(h)\right\|_{L^{2}(\Omega)^{3}} \frac{1}{h}\left\|\int_{0}^{h} u^{m}(s) \mathrm{d} s\right\|_{L^{2}(\Omega)^{3}} \\
& -\frac{1}{h} \int_{h}^{T}\left(\left(\rho^{m} u^{m}\right)(t), u^{m}(t)-u^{m}(t-h)\right) \mathrm{d} t \\
\leq & \frac{c_{1}}{h}\left(\int_{T-h}^{T} \mathrm{~d} s\right)^{1 / 2}\left(\int_{T-h}^{T}\left\|u^{m}(s)\right\|_{L^{2}(\Omega)^{3}}^{2} \mathrm{~d} s\right)^{1 / 2} \\
& \left.+\frac{c_{2}}{h}\left(\int_{0}^{h} \mathrm{~d} s\right)^{1 / 2}\left(\int_{0}^{h}\left\|u^{m}(s)\right\|_{L^{2}(\Omega)^{3}}^{2} \mathrm{~d} s\right)^{1 / 2}\right)^{1 / 2} \\
& -\frac{1}{h} \int_{h}^{T}\left(\left(\rho^{m} u^{m}\right)(t), u^{m}(t)-u^{m}(t-h)\right) \mathrm{d} t \\
\leq & \frac{c_{1}}{h} \sqrt{h}\left(\int_{0}^{T}\left\|u^{m}(s)\right\|_{L^{2}(\Omega)^{3}}^{2} \mathrm{~d} s\right)^{1 / 2}+\frac{c_{2}}{h} \sqrt{h} \int_{0}^{h}\left\|u^{m}(s)\right\|_{L^{2}(\Omega)^{3}}^{2} \mathrm{~d} s \\
& -\frac{1}{h} \int_{h}^{T}\left(\left(\rho^{m} u^{m}\right)(t), u^{m}(t)-u^{m}(t-h)\right) \mathrm{d} t \\
\leq & \frac{\tilde{c}_{1}}{h}\left(\int_{0}^{T}\left\|u^{m}(s)\right\|_{L^{2}(\Omega)^{3}}^{2} \mathrm{~d} s\right)^{1 / 2}-\frac{1}{h} \int_{h}^{T}\left(\left(\rho^{m} u^{m}\right)(t), u^{m}(t)-u^{m}(t-h)\right) \mathrm{d} t
\end{aligned}
$$

Portanto,

$$
\int_{h}^{T}\left(\frac{\partial}{\partial t} \rho^{m} u^{m}, \frac{1}{h} \int_{t-h}^{t} u^{m}(s) \mathrm{d} s\right) \mathrm{d} t \leq \frac{\tilde{c}_{1}}{\sqrt{h}}-\frac{1}{h} \int_{h}^{T}\left(\rho^{m}(t) u^{m}(t), u^{m}(t)-u^{m}(t-h)\right) \mathrm{d} t
$$

Observe-se agora que

$$
\begin{aligned}
& -\frac{1}{h} \int_{h}^{T}\left(\rho^{m} u^{m}(t), u^{m}(t)-u^{m}(t-h)\right) \mathrm{d} t \\
= & -\frac{1}{h} \int_{h}^{T} \int_{\Omega} \rho^{m} u^{m}(t)^{2} \mathrm{~d} x \mathrm{~d} t+\frac{1}{h} \int_{h}^{T} \int_{\Omega} \rho^{m}(t) u^{m}(t) u^{m}(t-h) \mathrm{d} x \mathrm{~d} t \\
= & \frac{1}{2 h} \int_{h}^{T} \int_{\Omega} \rho^{m}(t) u^{m}(t)^{2} \mathrm{~d} x \mathrm{~d} t+\frac{1}{2 h} \int_{h}^{T} \int_{\Omega} \rho^{m}(t) u^{m}(t-h) \mathrm{d} x \mathrm{~d} t \\
= & -\frac{1}{2 h} \int_{h}^{T} \int_{\Omega} \rho^{m}(t)\left(u^{m}(t-h)-u^{m}(t)\right)^{2} \mathrm{~d} x \mathrm{~d} t-\frac{1}{h} \int_{h}^{T} \int_{\Omega} \rho^{m}(t) u^{m}(t)^{2} \mathrm{~d} x \mathrm{~d} t \\
= & \frac{1}{2 h} \int_{h}^{T} \int_{\Omega} \rho^{m}(t) u^{m}(t-h)^{2} \mathrm{~d} x \mathrm{~d} t-\frac{1}{2 h} \int_{h}^{T} \int_{\Omega} \rho^{m}(t) u^{m}(t)^{2} \mathrm{~d} x \mathrm{~d} t \\
& -\frac{1}{2 h} \int_{h}^{T} \int_{\Omega} \rho^{m}(t)\left(u^{m}(t-h)-u^{m}(t)\right)^{2} \mathrm{~d} x \mathrm{~d} t .
\end{aligned}
$$

Nesta última expressão vamos somar e restar o termo

$$
\frac{1}{2 h} \int_{0}^{T} \int_{\Omega} \rho^{m}(t-h) u^{m}(t-h)^{2} \mathrm{~d} x \mathrm{~d} t
$$

e continuando com o cálculo acima, obtém-se

$$
=\frac{1}{2 h} \int_{h}^{T} \int_{\Omega} \rho^{m}(t-h) u^{m}(t-h)^{2} \mathrm{~d} x \mathrm{~d} t-\frac{1}{2 h} \int_{h}^{T} \int_{\Omega} \rho^{m}(t-h) u^{m}(t-h)^{2} \mathrm{~d} x \mathrm{~d} t
$$




$$
\begin{aligned}
& +\frac{1}{2 h} \int_{h}^{T} \int_{\Omega} \rho^{m}(t) u^{m}(t-h)^{2} \mathrm{~d} x \mathrm{~d} t-\frac{1}{2 h} \int_{h}^{T} \int_{\Omega} \rho^{m}(t) u^{m}(t)^{2} \mathrm{~d} x \mathrm{~d} t \\
& -\frac{1}{2 h} \int_{h}^{T} \int_{\Omega} \rho^{m}(t)\left(u^{m}(t-h)-u^{m}(t)\right)^{2} \mathrm{~d} x \mathrm{~d} t \\
= & \frac{1}{2 h} \int_{h}^{T} \int_{\Omega} \rho^{m}(t-h) u^{m}(t-h)^{2} \mathrm{~d} x \mathrm{~d} t \\
& -\frac{1}{2 h} \int_{h}^{T} \int_{\Omega} \rho^{m}(t) u^{m}(t)^{2} \mathrm{~d} x \mathrm{~d} t+\frac{1}{2 h} \int_{h}^{T}\left(\rho^{m}(t)-\rho^{m}(t-h)\right) u^{m}(t-h)^{2} \mathrm{~d} x \mathrm{~d} t \\
& -\frac{1}{2 h} \int_{h}^{T} \int_{\Omega} \rho^{m}(t)\left(u^{m}(t-h)-u^{m}(t)\right)^{2} \mathrm{~d} x \mathrm{~d} t .
\end{aligned}
$$

Fazendo a mudança de variáveis $\theta=t-h$, temos

$$
\frac{1}{2 h} \int_{h}^{T} \int_{\Omega} \rho^{m}(t-h) u^{m}(t-h)^{2} \mathrm{~d} x \mathrm{~d} t=\frac{1}{2 h} \int_{0}^{T-h} \rho^{m}(\theta) u^{m}(\theta)^{2} \mathrm{~d} x d \theta
$$

e, portanto, os dois primeiros termos podem ser reescritos como

$$
\begin{aligned}
& \frac{1}{2 h} \int_{0}^{T-h} \int_{\Omega} \rho^{m}(t) u^{m}(t)^{2} \mathrm{~d} x \mathrm{~d} t-\frac{1}{2 h} \int_{h}^{T} \int_{\Omega} \rho^{m}(t) u^{m}(t)^{2} \mathrm{~d} x \mathrm{~d} t \\
= & \frac{1}{2 h} \int_{0}^{h} \int_{\Omega} \rho^{m}(t)\left(u^{m}(t)^{2} \mathrm{~d} x \mathrm{~d} t+\frac{1}{2 h} \int_{T-h}^{T} \int_{\Omega} \rho^{m}(t) u^{m}(t)^{2} \mathrm{~d} x \mathrm{~d} t .\right.
\end{aligned}
$$

Observemos

$$
\begin{aligned}
\left|\int_{0}^{h} \int_{\Omega} \rho^{m}(t) u^{m}(t)^{2} \mathrm{~d} x \mathrm{~d} t\right| & \leq \int_{0}^{h}\left\|\left(\rho^{m}\right)^{1 / 2} u^{m}\right\|_{L^{2}(\Omega)^{3}}\left\|u^{m}\right\|_{L^{2}(\Omega)^{3}} \mathrm{~d} t \\
& \leq C_{0}\left\|u^{m}\right\|_{L^{2}(\Omega)^{3}} \leq C_{0}\left(\int_{0}^{h} \mathrm{~d} t\right)^{1 / 2}\left(\int_{0}^{h}\left\|u^{m}\right\|_{L^{2}(\Omega)^{3}}^{2}\right)^{1 / 2} \\
& \leq C_{0} \sqrt{h}\left(\int_{0}^{T}\left\|u^{m}(t)\right\|^{2} \mathrm{~d} t\right)^{1 / 2}
\end{aligned}
$$

é o que $\left(\rho^{m}\right)^{1 / 2} u^{m} \in L^{\infty}(0, T ; H)$.

Similarmente, obtém-se

$$
\frac{1}{2 h} \int_{0}^{T-h} \int_{\Omega} \rho^{m}(t) u^{n}(t)^{2} \mathrm{~d} x \mathrm{~d} t-\frac{1}{2 h} \int_{h}^{T} \int_{\Omega} \rho^{m}(t) u^{m}(t)^{2} \mathrm{~d} x \mathrm{~d} t \leq \frac{\tilde{C}_{2}}{\sqrt{h}} .
$$

Para maior clareza, faremos o cálculo a seguir utilizando os sub-índices das coordenadas. Portanto, integrando a equação da densidade (4.26) obtém-se

$$
\rho^{m}(t)-\rho^{m}(t-h)=\sum_{j=1}^{3} \int_{t-h}^{t} u^{m}(s) \frac{\partial \rho^{m}}{\partial x_{j}} \mathrm{~d} s .
$$


Multiplicando esta última expressão por $u^{m}(t-h)^{2}$ e integrando sobre $\Omega$, obtém-se

$$
\begin{aligned}
& \int_{\Omega}\left[\rho^{m}(t)-\rho^{m}(t-h)\right] u^{m}(t-h)^{2} \mathrm{~d} x \\
= & \sum_{j=1}^{3} \int_{\Omega}\left(\int_{t-h}^{t} u_{j}^{m}(s) \frac{\partial \rho^{m}}{\partial x_{j}}(s) \mathrm{d} s\right) u_{m}(t-h)^{2} \mathrm{~d} x \\
= & \sum_{j=1}^{3} \int_{\Omega} \frac{\partial}{\partial x_{j}}\left(\int_{t-h}^{t} u_{j}^{m}(s) \rho^{m}(s) \mathrm{d} s\right) u^{m}(t-h)^{2} \mathrm{~d} x \\
= & 2 \sum_{i, j=1}^{2} \int_{\Omega}\left(\int_{t-h}^{t} u_{j}^{m}(s) \rho^{m}(s) \mathrm{d} s\right) u_{i}^{m}(t-h) \frac{\partial}{\partial x_{j}} u_{i}^{m}(t-h) \mathrm{d} x .
\end{aligned}
$$

Portanto, integrando em relação a $t$, de $h$ até $T$, obtemos

$$
\begin{aligned}
& \int_{h}^{T} \int_{\Omega}\left(\rho^{m}(t)-\rho^{m}(t-h)\right) u^{m}(t-h)^{2} \mathrm{~d} x \\
= & 2 \sum_{i, j=1}^{3} \int_{h}^{T} \int_{\Omega}\left(\int_{t-h}^{t} u^{m}(s) \rho^{m}(s) \mathrm{d} s\right)_{j} u_{i}^{m}(t-h) \frac{\partial}{\partial x_{j}} u_{i}^{m}(t-h) \mathrm{d} x \mathrm{~d} t .
\end{aligned}
$$

Se aplicarmos desigualdade de Hölder e voltando para a notação anterior, obtémse

$$
\begin{aligned}
& \mid \int_{h}^{T}\left[\int_{\Omega}\left(\rho^{m}(t)-\rho^{m}(t-h)\right) u^{m}(t-h)^{2}\right] \mathrm{d} x \mathrm{~d} t \\
\leq & \int_{h}^{T}\left(\left\|u^{m}(t-h)\right\|_{L^{4}(\Omega)^{3}}\left\|\nabla u^{m}(t-h)\right\|_{L^{2}(\Omega)^{9}}\left\|\int_{t-h}^{t} u^{m}(s) \rho^{m}(s) \mathrm{d} s\right\|_{L^{4}(\Omega)^{3}}\right. \\
\leq & \int_{h}^{T}\left(\left\|\nabla u^{m}(t-h)\right\|_{L^{2}(\Omega)^{9}}^{2} \cdot \int_{t-h}^{t}\left\|\rho^{m}(s) u^{m}(s)\right\|_{L^{4}(\Omega)^{3}} \mathrm{~d} s\right) \mathrm{d} t \\
\leq & C_{3} \int_{h}^{T}\left\|\nabla u^{m}(t-h)\right\|_{L^{2}(\Omega)^{9}}^{2}\left(\int_{t-h}^{t} \mathrm{~d} s\right)^{1 / 2}\left(\int_{t-h}^{t}\left\|u^{m}(s)\right\|_{L^{4}(\Omega)^{3}}^{2} \mathrm{~d} s\right)^{1 / 2} \mathrm{~d} t \\
\leq & C_{3} \sqrt{h} \int_{h}^{T}\left\|\nabla u^{m}(t-h)\right\|_{L^{2}(\Omega)^{9}}^{2} \int_{0}^{T}\left\|\nabla u^{m}(s)\right\|_{L^{2}(\Omega)^{9}}^{2} \mathrm{~d} s \mathrm{~d} t \\
\leq & \tilde{C}_{3} \sqrt{h} .
\end{aligned}
$$

Portanto, deduzimos que

$$
\frac{1}{2 h} \int_{h}^{T} \int_{\Omega}\left(\rho^{m}(t)-\rho^{m}(t-h) u^{m}(t-h)^{2} \mathrm{~d} x \mathrm{~d} t \leq \frac{\tilde{C}_{3}}{\sqrt{h}} .\right.
$$

Em conclusão, usando (4.66), (4.67), (4.68), tem-se

$$
\int_{h}^{T}\left(\frac{\partial}{\partial t} \rho^{m} u^{m}, \frac{1}{h} \int_{t-h}^{t} u^{m}(s) \mathrm{d} s\right)
$$




$$
\begin{aligned}
\leq & \frac{\tilde{c}}{\sqrt{h}}\left(\int_{0}^{T}\left|u^{m}(s)\right|^{2} \mathrm{~d} s\right)^{1 / 2}-\frac{1}{h} \int_{h}^{T}\left(\rho^{m}(t) u^{m}(t), u^{m}(t)-u^{m}(t-h)\right) \mathrm{d} t \\
\leq & \frac{\tilde{c}}{\sqrt{h}}\left(\int_{0}^{T}\left|u^{m}(s)\right|^{2} \mathrm{~d} s\right)^{1 / 2}+\frac{1}{2 h} \int_{h}^{T} \int_{\Omega} \rho^{m}(t-h) u^{m}(t-h)^{2} \mathrm{~d} x \mathrm{~d} t \\
& -\frac{1}{2 h} \int_{h}^{T} \int_{\Omega} \rho^{m}(t) u^{m}(t)^{2} \mathrm{~d} x \mathrm{~d} t-\frac{1}{2 h} \int_{h}^{T} \int_{\Omega}\left(\rho^{m}(t)-\rho^{m}(t-h)\right) u^{m}(t-h)^{2} \mathrm{~d} x \mathrm{~d} t \\
& -\frac{1}{2 h} \int_{h}^{T}\left(\int_{\Omega} \rho^{m}(t)\left(u^{m}(t-h)-u^{m}(t)\right)^{2} \mathrm{~d} x \mathrm{~d} t\right. \\
\leq & \frac{\tilde{C}_{1}}{\sqrt{h}}+\frac{\tilde{C}_{2}}{\sqrt{h}}+\frac{\tilde{C}_{3}}{\sqrt{h}}-\frac{1}{2 h} \int_{h}^{T}\left|\sqrt{\rho^{m}(t)}\left(u^{m}(t-h)-u^{m}(t)\right)\right|^{2} \mathrm{~d} t .
\end{aligned}
$$

Portanto,

$$
\int_{h}^{T}\left(\frac{\partial}{\partial t} \rho^{m} u^{m}, \frac{1}{h} \int_{t-h}^{t} u^{m}(s) \mathrm{d} s \leq \frac{C}{\sqrt{h}}-\frac{1}{2 h} \int_{h}^{T} \mid \sqrt{\rho^{m}(t)}\left(u^{m}(t-h)-u^{m}(t)\right)^{2} \mathrm{~d} t .\right.
$$

Vejamos agora o segundo termo da equação (4.65); observemos que

$$
\begin{aligned}
& \int_{h}^{T}\left|\rho^{m}\left(u^{m} \cdot \nabla\right) u^{m} u^{m} \mathrm{~d} x\right| \mathrm{d} t \leq C \int_{h}^{T}\left\|u^{m}(t)\right\|_{L^{4}(\Omega)^{3}}\left\|\nabla u^{m}\right\|\left\|u_{h m}\right\|_{L^{4}(\Omega)^{3}} \\
\leq & C \int_{0}^{T}\left\|\nabla u^{m}(t)\right\|_{L^{2}(\Omega)^{3}}^{2}\left\|\frac{1}{h} \int_{t-h}^{t} u^{m}(s) \mathrm{d} s\right\|_{L^{4}(\Omega)^{3}} \\
\leq & C \int_{h}^{T}\left(\left\|\nabla u^{m}(t)\right\|_{L^{2}(\Omega)^{3}}^{2} \frac{1}{h} \int_{t-h}^{t}\left\|u^{m}(s)\right\|_{L^{4}(\Omega)^{3}} \mathrm{~d} s\right) \mathrm{d} t \\
\leq & C \int_{0}^{T}\left(\left\|\nabla u^{m}(t)\right\|_{L^{2}(\Omega)^{9}}^{2} \frac{1}{h}\left(\int_{t-h}^{t} \mathrm{~d} s\right)^{1 / 2}\left(\int_{t-h}^{t}\left\|\nabla u^{m}(s)\right\|_{L^{2}(\Omega)^{9}}^{2} \mathrm{~d} s\right)^{1 / 2} \mathrm{~d} t\right. \\
\leq & \frac{C}{\sqrt{h}} \int_{h}^{T}\left(\left\|\nabla u^{m}(t)\right\|_{L^{2}(\Omega)^{9}}^{2} \int_{t-h}^{t}\left\|\nabla u^{m}(s)\right\|_{L^{2}(\Omega)^{9}}^{2} \mathrm{~d} s\right)^{1 / 2} \mathrm{~d} t \\
\leq & \frac{C}{\sqrt{h}} \int_{h}^{T}\left(\left\|\nabla u^{m}(t)\right\|_{L^{2}(\Omega)^{3}}^{2}\left(\int_{h}^{T}\left\|\nabla u^{m}(s)\right\|^{2} \mathrm{~d} s\right)^{1 / 2} \mathrm{~d} t\right. \\
\leq & \frac{\tilde{C}_{4}}{\sqrt{h}} .
\end{aligned}
$$

Agora limitaremos o termo $\left(\mu+\mu_{r}\right)$ a $\left(u^{m}, u_{h m}\right)$ em (4.65). Notemos que

$$
\begin{aligned}
& \left(\mu+\mu_{r}\right) \int_{h}^{T} a\left(u^{m}, u_{h m}\right) \mathrm{d} t=\left(\mu+\mu_{r}\right) \int_{h}^{T}\left\|\nabla u^{m}(t)\right\|_{L^{2}(\Omega)^{9}}\left\|\nabla u_{h m}(t)\right\|_{L^{2}(\Omega)^{9}} \mathrm{~d} t \\
= & \left(\mu+\mu_{r}\right) \int_{h}^{T}\left\|\nabla u^{m}(t)\right\|_{L^{2}(\Omega)^{9}}\left\|\nabla \frac{1}{h} \int_{t-h}^{t} u^{m}(s) \mathrm{d} s\right\|_{L^{2}(\Omega)^{9}} \\
= & \left(\mu+\mu_{r}\right) \int_{h}^{T}\left\|\nabla u^{m}(t)\right\| \|_{L^{2}(\Omega)^{9}} \frac{1}{h}\left(\int_{t-h}^{t}\left\|\nabla u^{m}(s)\right\|_{L^{2}(\Omega)^{9}} \mathrm{~d} s\right) \mathrm{d} t \\
\leq & \left(\mu+\mu_{r}\right) \int_{h}^{T}\left\|\nabla u^{m}(t)\right\| \|_{L^{2}(\Omega)^{9}} \frac{1}{\sqrt{h}}\left(\int_{h}^{T}\left\|\nabla u^{m}(s)\right\|_{L^{2}(\Omega)^{9}}^{2} \mathrm{~d} s\right)^{1 / 2} \mathrm{~d} t
\end{aligned}
$$




$$
\begin{aligned}
& \leq \frac{C_{6}}{\sqrt{h}} \int_{h}^{T}\left\|\nabla u^{m}(t)\right\| \|_{L^{2}(\Omega)^{9}}\left(\int_{h}^{T}\left\|\nabla u^{m}(s)\right\|_{L^{2}(\Omega)^{9}}^{2} \mathrm{~d} s\right) \mathrm{d} t \\
& \leq \frac{\tilde{C}_{6}}{\sqrt{h}} .
\end{aligned}
$$

Logo,

$$
\left(\mu+\mu_{r}\right) \int_{h}^{T} a\left(u^{m}, u_{h m}\right) \mathrm{d} t \leq \frac{\tilde{C}_{6}}{\sqrt{h}}
$$

Agora, o outro termo pode ser limitado como

$$
\begin{aligned}
2 \mu_{r} \int_{h}^{T}\left(\operatorname{rot} w_{n}, u_{h m}\right) \mathrm{d} t & \leq 2 \mu_{r} \int_{h}^{T}\left\|w_{n}\right\|_{L^{2}(\Omega)^{3}}\left\|\operatorname{rot} u_{h m}\right\|_{L^{2}(\Omega)^{3}} \mathrm{~d} t \\
& \leq 2 \mu_{r} \int_{h}^{T}\left\|w_{n}\right\|_{L^{2}(\Omega)^{9}}\left\|\nabla u_{h m}\right\|_{L^{2}(\Omega)^{9}} \mathrm{~d} t \\
& \leq 2 \mu_{r} \int_{h}^{T}\left\|w^{m}(t)\right\|_{L^{2}(\Omega)^{3}} \frac{1}{h}\left(\int_{t-h}^{t}\left\|\nabla u^{m}(s)\right\| \|_{L^{2}(\Omega)^{9}} \mathrm{~d} s\right) \mathrm{d} t \\
& \leq \frac{2 \mu_{r}}{\sqrt{h}} \int_{h}^{T}\left\|w^{m}(t)\right\| \|_{L^{2}(\Omega)^{3}}\left(\int_{t-h}^{t}\left\|\nabla u^{m}(s)\right\|_{L^{2}(\Omega)^{9}}^{2} \mathrm{~d} s\right)^{1 / 2} \mathrm{~d} t \\
& \leq \frac{2 \mu_{r}}{\sqrt{h}} \int_{h}^{T}\left\|w_{n}(t)\right\|_{L^{2}(\Omega)^{3}}\left(\int_{h}^{T}\left\|\nabla u^{m}(s)\right\|_{L^{2}(\Omega)^{9}}^{2} \mathrm{~d} s\right)^{1 / 2} \mathrm{~d} t \\
& \leq \frac{\tilde{C}_{7}}{\sqrt{h}} .
\end{aligned}
$$

Observemos agora que

$$
\begin{aligned}
& m \int_{h}^{T}\left(u^{m}(t)-P_{k}^{1} u^{m}(t), \frac{1}{h} \int_{t-h}^{t} u^{m}(s) \mathrm{d} s\right) \mathrm{d} t=m \int_{h}^{T}\left(u^{m}(t)-P_{k}^{1} u^{m}(t),\right. \\
& \left.\frac{1}{h} \int_{t-h}^{t}\left(u^{m}(s)-P_{k}^{1} u^{m}(s)\right) \mathrm{d} s\right) \mathrm{d} t+m \int_{h}^{T}\left(u^{m}(t)-P_{k}^{1} u^{m}(t),\right. \\
& \left.\frac{1}{h} \int_{t-h}^{t} P_{k}^{1} u^{m}(s) \mathrm{d} s-P_{k}^{1} u^{m}(t)\right) \mathrm{d} t+m \int_{h}^{T}\left(u^{m}(t)-P_{k}^{1} u^{m}(t), P_{k}^{1} u^{m}(t)\right) \mathrm{d} t .
\end{aligned}
$$

Observando agora que $\frac{1}{h} \int_{t-h}^{t} P_{k}^{1} u^{m}(s) \mathrm{d} s \in \mathbb{K}_{1}$ pois $0 \leq\left(v-P_{k}^{1} v, P_{k}^{1} v-h\right)$ $\forall h \in \mathbb{K}_{1} \mathrm{e}$

$$
\left.m \int_{h}^{T}\left(u^{m}(t)-P_{k}^{1} u^{m}(t), \frac{1}{h} \int_{t-h}^{t} P_{k}^{1} u^{m}(s) \mathrm{d} s-P_{k}^{1} u^{m}(t)\right)\right) \mathrm{d} t \leq 0
$$

e como

$$
m \int_{h}^{T}\left(u^{m}(t)-P_{k}^{1} u^{m}(t), P_{k}^{1} u^{m}(t)\right) \leq C
$$


finalmente, obtém-se de (4.65) que

$$
0 \leq \frac{\tilde{C}_{10}}{\sqrt{h}}-\frac{1}{h} \int_{h}^{T} \mid \sqrt{\rho^{m}(t)}\left(u^{m}(t)-\left.u^{m}(t-h)\right|^{2} \mathrm{~d} t\right.
$$

e portanto

$$
\int_{0}^{T-h}\left|\sqrt{\rho^{m}(t+h)}\left(u^{m}(t+h)-u^{m}(t)\right)\right|^{2} \mathrm{~d} t \leq C \sqrt{h} .
$$

Similarmente, considerando $w_{h m}=\frac{1}{h} \int_{t-h}^{t} w^{m}(s) \mathrm{d} s$ e substituindo $w_{h m}$ por $\phi_{k}$ em (4.25), obtém-se a equação

$$
\begin{aligned}
& \left(\rho^{m} \frac{\partial w_{n}}{\partial t}, w_{h m}\right)+\left(\rho^{m}\left(u^{m} \cdot \nabla w_{h m}, w_{h m}\right)+B\left(w^{m}, w_{h m}\right)+m\left(Q^{2}\left(w^{m}\right), w_{h m}\right)\right. \\
& -2 \mu_{r}\left(\operatorname{rot}\left(u^{m}\right), w_{h m}\right)+4 \mu_{r}\left(w^{m}, w_{h m}\right)=\left(\rho^{m} g, w_{h m}\right) .
\end{aligned}
$$

$\mathrm{O}$ mesmo argumento permite mostrar que

$$
\int_{0}^{T-h}\left|\sqrt{\rho^{m}(t+h)}\left(w^{m}(t+h)-w^{m}(t)\right)\right|^{2} \mathrm{~d} t \leq C_{0} \sqrt{h}
$$

e portanto obtivemos as estimativas (4.61) e (4.62). Sejam $\phi, \psi$ vetores quaisquer nos espaços $\Phi$ e $\Psi$ respectivamente tal que $\phi \in \mathbb{K}_{1}$ e $\psi \in \mathbb{K}_{2}$. Além disso, sejam $\phi^{m}(t), \psi^{m}(t)$ as suas projeções em $V^{m}$ e $W^{m}$ respectivamente. Como

$$
\begin{aligned}
& \phi^{m}(t) \rightarrow \phi(t) \text { forte no } L^{2}\left(Q_{T}\right)^{3}, \\
& \psi^{m}(t) \rightarrow \psi(t) \text { forte no } L^{2}\left(Q_{T}\right)^{3},
\end{aligned}
$$

temos que $\phi(t) \in \mathbb{K}_{1}$ e $\psi(t) \in \mathbb{K}_{2}$, portanto existe $m_{0}$ tal que $\forall m>m_{0}$ e se $m>\bar{m}>m_{0}$ temos

$$
\begin{aligned}
& \int_{0}^{T}\left\{\left(\frac{\partial}{\partial t}\left(\rho^{m} u^{m}\right), \phi^{m}-u^{m}\right)+\left(\rho u^{m} \cdot \nabla u^{m}, \phi^{m}-u^{m}\right)+\left(\mu+\mu_{r}\right) a\left(u^{m}, \phi^{m}-u^{m}\right)\right. \\
& +m\left(Q^{1}\left(u^{m}\right), \phi^{m}-u^{m}\right)+2 \mu_{r}\left(\operatorname{rot}\left(w^{m}\right), \phi^{m}-u^{m}\right)-\left(\rho^{m} f, \phi^{m}-u^{m}\right)=0
\end{aligned}
$$

$\mathrm{e}$

$$
\begin{aligned}
& \int_{0}^{T}\left\{\left(\frac{\partial}{\partial t}\left(\rho^{m} w^{m}\right), \phi^{m}-w^{m}\right)+\left(\rho^{m} u^{m} \cdot \nabla w^{m}, \psi^{m}-w^{m}\right)+B\left(w^{m}, \psi^{m}-w^{m}\right)\right. \\
& +m\left(Q^{2}\left(w^{m}\right), \psi^{m}-w^{m}\right)+4 \mu_{r}\left(w^{m}, \psi^{m}-w^{m}\right)-2 \mu_{r}\left(\operatorname{rot} u^{m}, \psi^{m}-w^{m}\right) \\
= & \left(\rho^{m} f, \psi^{m}-w^{m}\right) .
\end{aligned}
$$


Como

$$
\begin{aligned}
m\left(Q^{1}\left(u^{m}\right), \phi^{m}-u^{m}\right) & \leq 0 \\
m\left(Q^{2}\left(w^{m}\right), \psi^{m}-w^{m}\right) & \leq 0
\end{aligned}
$$

obtemos que

$$
\begin{aligned}
& \int_{0}^{T}\left\{\left(\frac{\partial}{\partial t}\left(\rho^{m} u^{m}\right), \phi^{m}-w^{m}\right)+\left(\rho^{m} u^{m} \cdot \nabla w^{m}, \phi^{m}-u^{m}\right)+\left(\mu+\mu_{r}\right) a\left(u^{m}, \phi^{m}-u^{m}\right)\right. \\
& +2 \mu_{r}\left(\operatorname{rot} w^{m}, \phi^{m}-u^{m}\right)-\left(\rho^{m} f, \phi^{m}-u^{m}\right) \geq 0
\end{aligned}
$$

$\mathrm{e}$

$$
\begin{aligned}
& \int_{0}^{T}\left\{\left(\frac{\partial}{\partial t}\left(\rho^{m} w^{m}\right), \phi^{m}-w^{m}\right)+\left(\rho^{m} u^{m} \cdot \nabla w^{m}, \psi^{m}-w^{m}\right)+B\left(w^{m}, \psi^{m}-w^{m}\right)\right. \\
& +4 \mu_{r}\left(w^{m}, \psi^{m}-w^{m}\right)-2 \mu_{r}\left(\operatorname{rot} u^{m}, \psi^{m}-w^{m}\right)-\left(\rho^{m} g, \psi^{m}-w^{m}\right) \geq 0 .
\end{aligned}
$$

Fazendo procedimento análogo ao que foi feito para obter as formulações variacionais, obtém-se

$$
\begin{aligned}
& \int_{0}^{T}\left\{\left(\rho^{m} \frac{\partial \phi^{m}}{\partial t}, \phi^{m}-u^{m}\right)+\left(\rho^{m} u^{m} \cdot \nabla \phi^{m}, \phi^{m}-u^{m}\right)+\left(\mu+\mu_{r}\right) a\left(u^{m}, \phi^{m}-u^{m}\right)\right. \\
& +2 \mu_{r}\left(\operatorname{rot} w^{m}, \phi^{m}-u^{m}\right) \\
\geq & \left(\rho^{m} f, \phi^{m}-u^{m}\right)-\frac{1}{2}\left\|\left(\rho^{m}(0)\right)^{1 / 2}\left(\phi^{m}(0)-u^{m}(0)\right)\right\|^{2}
\end{aligned}
$$

e

$$
\begin{aligned}
& \int_{0}^{T}\left\{\left(\rho^{m} \frac{\partial \psi^{m}}{\partial t}, \psi^{m}-w^{m}\right)+\left(\rho^{m} u^{m} \cdot \nabla w^{m}, \psi^{m}-w m\right)+B\left(w^{m}, \psi^{m}-w^{m}\right)\right. \\
& \left.+4 \mu_{r}\left(w^{m}, \psi^{m}-w^{m}\right)-2 \mu_{r}\left(\operatorname{rot} u^{m}, \psi^{m}-w^{m}\right)-\left(\rho^{m} g, \psi^{m}-w^{m}\right)\right\} \mathrm{d} t \\
\geq & -\frac{1}{2}\left\|\left(\rho^{m}(0)\right)^{1 / 2}\left(\psi^{m}(0)-w^{m}(0)\right)\right\|^{2} .
\end{aligned}
$$

Usando o procedimento do limite em $m$, obtém-se que $(u, \rho, w)$ satisfazem (a), (b), (c), (d). Portanto, demonstramos o teorema principal. 


\subsection{Problemas em aberto}

Nesta última seção gostaríamos de colocar algumas questões que nos parecem importantes para um estudo posterior.

(1) Como é bem conhecido nas equações de Navier-Stokes a unicidade das soluções fracas num domínio $\Omega$ dentro de $\mathbb{R}^{3}$ é um problema em aberto. Porém, existem alguns resultados que nos permitem deduzir a regularidade (e conseqüentemente a unicidade) se colocarmos condicões extras sobre a solução $u$, são os chamados resultados, conhecidos como criterios de regularidade de Serrin (ver Serrin [77], Lions [43], Von Whal [86]). Como estender estes critérios ao sistema estudado neste trabalho?

Outro caminho é colocar condições sobre a pressão para obter a regularidade. Esta idéia é devida inicialmente a Kaniel [32] e desenvolvida mais recentemente por Beirão da Veiga [3], [4], [5], Berselli [6], etc. Como estender estas idéias ao modelo estudado neste trabalho?

(2) Será possível estudar o modelo estacionário associado a este modelo? Observamos que mesmo no caso mais simples, onde não temos rotação (não tem $w$ ), o problema tem sido pouco estudado, veja Frolov [25], [26] e Santos [74]. Convém salientar que o estudo do modelo estacionário é mais dificil que o modelo de evolução.

(3) Será possível fazer um estudo via operadores de evolução para o modelos deste trabalho? Observamos que no caso não-homogêneo tal estudo foi feito por Okamoto [54] no contexto $L^{2}$, porém um estudo no contexto $L^{p}$ ainda sido feito.

(4) Existência de soluções periódicas fortes também é outra possibilidade de estu- 
do.

(5) Comportamento assintótico das soluções quando $t$ vai parao o infinito, mesmo no caso não-homogêneo não tem sido estudado.

(6) Um estudo do modelo em domínios estreitos (Thin dominios) também é um aspecto que mereceria ser desenvolvido.

(7) Questões numéricas associadas ao modelo proposto seriam interessantes de desenvolver. Este é um campo virgem de pesquisa.

(8) Estudar o modelo associado em domínios que variam com o tempo. Devemos dizer que isto está sob estudo. De fato, já temos praticamente um trabalho pronto.

(9) Estudar solucões fortes e a eventual unicidade da desigualdade variacional estudada no Capítulo 4.

(10) Estudar problemas de controle onde as restrições dinâmicas vêm dadas pelo sistema estudado neste trabalho.

(11) Fazer um estudo em domínios exteriores.

(12) O problema de Cauchy associado ao modelo estudado neste trabalho.

(13) Qual será o comportamento da solução do sistema se as viscosidades convergem para zero? Será que a solução converge à solução de um fluido micropolar ideal (ou de Euler)?

(14) Como são as explosões da solução forte? 
(15) As mesmas perguntas feitas para as equações de Navier-Stokes clássicas poderiam ser feitas para este modelo. Assim, inúmeras questões poderiam ser colocadas. 


\section{Referências}

[1] Adams, R. A. Sobolev Spaces, Academic Press, New York, 1975.

[2] Antonzev, S. N., Kazhikov, A. V., Monakhov, V. N., Boundary Value Problems in Mechanics of Nonhomogeneous Fluids, Norht-Holland, Amsterdam, 1990.

[3] Beirão da Veiga, H., A new regularity class for the Navier-Stokes equations in IR ${ }^{n}$, Chin. Ann. of Math., 16B: 4(1995), 407-412.

[4] Beirão da Veiga, H., Concerning the regularity of the solutions to the NavierStokes equations via the truncation method, Part I, Differential and integral Equations, 10 (1997), 1149-1156.

[5] Beirão da Veiga, H., On the smoothness of a class of weak solutions to the Navier-Stokes equations, J. Math. Fluid Mech., 2 (2000), 315-323.

[6] Berselli, L., Sufficient conditions for the regularity of the solutions of the NavierStokes Equations, Math. Meth. Appl. Sci., 22 (1999), 1079-1085.

[7] Binoli, M., Sur l'inéquation d'evolution de Navier-Stokes III, Atti, 52 (1972), 811-820.

[8] Boldrini, J. L., Rojas-Medar, M. A., On the convergence rate of spectral approximation for the equations for nonhomogeneous asymmetric fluids, Math. Mod. Num. Anal. (M²AN) 30 (1996), 123-155.

[9] Boldrini, J. L., Rojas-Medar, J., Global strong solutions of the equations for the motion of nonhomogeneous incompressible fluids, Numerical Methods in $\mathrm{Me}$ chanics (eds. C. Conca, G. N. Gática), Pitman Research Note in Mathematics Series, vol. 371 (1997), 35-45.

[10] Boldrini, J. L., Rojas-Medar, M., Strong Solutions of the Equations for Nonhomogeneous Asymetric Fluids, R-P 21/98, IMECC-UNICAMP, 1998, submetido a publicação. 
[11] Born, M., Über di beweglichkeit der electrolytischen Ionen, Zeitschrift für das Physik 1 (1920), 221-235.

[12] Cattabriga, L., Su un problema al contorno relativo al sistema di equazioni di Stokes, Rend. Sem. Math. Univ. Padova, 31 (1961), 308-340.

[13] Condiff, D. W., Dalher, J. S., Fluid mechanics of antisymmetric stress, Phys. Fluids 11 (1964), 842-854.

[14] Conca, C., Gormaz, R., Ortega-Torres, E. E., Rojas-Medar, M. A. Existence and uniquess of strong solution for nonhomogeneous micropolar fluids, Nonlinear Partial Differential Equations and Their Applications (eds. J. L. Lions, C. Cionarescu), Pitman XIV (2000).

[15] Courant, R., Hilbert, D., Methods of Mathematical Physics, vol. II (1962).

[16] Dalher, J. S., Scriven, L. E., Theory of structured continua. 1. General consideration of angular momentum and polarization, Proc. Roy. Soc. (London) A275 (1963), 504-527.

[17] Durán, M., Ferreira, J., Rojas-Medar, M. A., Reproductive weak solutions for magneto-micropolar fluid motion, aceito para publicação em Math. Comp. Modelling.

[18] Durán, M., Ortega-Torres, E. E., Rojas-Medar, M. A., Stationary solutions of magneto-micropolar equations in exterior domains, Technical Report, $\mathrm{n}$. 20/98, IMECC-UNICAMP (1998), submetido a publicação.

[19] Duvaut, G., Lions, J. L., Les Inequations en Mécanique et en Physique, Dunod, Pari, 1972.

[20] Eringen, A. C., Theory of micropolar fluids, J. Math. Mech. 16 (1966), 1-8.

[21] Eringen, A. C., Simple microfluids, Int. J. Enging. Sci, 2 (1964), 205-217.

[22] Eringen, A. C., Theory of anysotropic micropolar fluids, Int. J. Enging. Sci. 18 (1980), 5-17.

[23] Eringen, A. C., Micropolar fluids with stretch, Int. J. Enging. Sci. 7 (1960), 115-127. 
[24] Fernández-Caras, E., Guillen, F., The existence of nonhomogeneous, viscous and incompressible flow in unbounded domain, Comm. P. D. E. 17 (1992), 125.3-1265.

[25] Frolov, N. N. Solvability of a boundary problem of motion of an inhomogeneous fluid, Math. Zametki, 53 (6), (1993), 130-140.

[26] Frolov, N. N. Boundary value problem describing the motion of an inhomogeneous fluids, Siberian Math. J., 37, (1996), 376-393.

[27] Galdi, P., An Introduction to the Mathematical Theory of the Navier-Stokes Equations, v.1., Springer-Verlag (1997).

[28] Girault, V., Raviart, P. A., Finite element approximation of the Navier-Stokes equations, Lecture Notes in Math., v.749, Springer-Verlag, Berlin, 1979.

[29] Glowinsky, R., Lions, J. L., Trémolières, R., Numerical Analysis of Variational Inequalities, North-Holland, 1976.

[30] Heywood, J. G., The Navier-Stokes equations: on the existence regularity and decay of solutions, Ind. Univer. Math. Journal, 25(5) (1980), 639-681.

[31] Itoh, S., Tani, A., Solvability of nonstationary problems for nonhomogeneous inompressible fluids and the convergence with vanishing viscosity, Tokyo $J$. Math., 22 (1999), 17-42.

[32] Kaniel, S., A sufficient conditions for smoothness of solutions of Navier-Stokes equations, Israel J. Math., 6 (1968), 354-358.

[33] Kaniel, S., Shinbrot, M., A reproductive property of the Navier-Stokes equations, Arch. Rational. Mech. Anal. 24 (1967), 363-369.

[34] Kato, H., Existence of periodic solutions of the Navier-Stokes equations, $J$. Math. Anal. and Appl., 208 (1997), 141-157.

[35] Khoi, V., Schmitt, K., On the variational inequalities associated with the Navier-Stokes equations; some bifurcations problems, Differential Equations and Computational Simulations III, Electronic Journal of Differencital Equations Conference, (1997), 137-148.

[36] Kim, J. U., Weak solutions of an initial boundary-value problem for an incompressible viscous fluid with non-negative density, SIAM J. Math. Anal., 18(1) (1987), 89-96. 
[37] Kufner, A., John, O., Fučik, S., Funtion Spaces, Noordhoff International Publishing, 1977.

[38] Ladyzhenskaya, O. A., The Mathematical Theory of Viscous Incompressible Fluid, Gordon and Beach, 1969).

[39] Ladyzhenskaya, O. A., The Boundary Value Problems of Mathematical Physics, Springer-Verlag, 1985.

[40] Ladyzhenskaya, O. A., Solonnikov, V. A., Ural'Ceva linear and quasilinear equations of parabolic type, Transl. Mathem. Monographs, v.23, AMS (1968).

[41] Ladyzhenskaya, O. A., Solonnikov, V. A., Unique solvability of an initial and boundary value problem for viscous incompressible nonhomogeneous fluids, $J$. Soviet Math. 9 (1978), 697-749.

[42] Lemoine, J., On non-homogeneous viscous incompressible fluids. Existence of regular solutions, Comment. Math. Univ. Carolinae 38, 4 (1997), 697-715.

[43] Lions, J. L., Quelques Méthodes de Résolution des Problémes aux Limits non Linéares, Dumond, Paris, 1969.

[44] Lions, J. L., On Some Problems Connect with Navie-Stokes Equations, Nonlinear Evolutions Equations, Academic Press, New York, 1978.

[45] Lions, P. L., Mathematical Topics in Fluid Mechanics, v.1, Incompressible Models, Clarendon Press, Oxford, 1996.

[46] Lukaszewicz, G., On the nonstationary flows of a simmetric fluids, Rend. Accad. Naz. Memoire di Matematica, v.12 (1988), 83-97.

[47] Lukaszewicz, G., On the existence, uniqueness and asymptotic properties for solutions of flows of asymetric fluids, Rend. Accad. Naz. Sci., Memoire di Matematica, v.13 (1989), 27-31.

[48] Lukaszewicz, G., Micropolar Fluids: Theory and Applications, Birkhauser, Berlin, 1998.

[49] Lukaszewics, G., On nonstationary flows of incompressible asymmetric fluids, Math. Meth. in the Appl. Sci., 13 (1990), 219-232. 
[50] Lukaszewicz, G., Ortega-Torres, E. E., Rojas-Medar, M. A., Strong periodic solutions for a class of abstract solutions equations, Technical Report RW 0100 (86), Institute of Applied Math. and Mech. Warsaw University (2001), submetido a publicação.

[51] Lukaszewicz, G., Rojas-Medar, M. A., Santos, M., Stationary micropolar fluid with boundary data in $L^{2}$. Aceito para publicação em J. Math. Anal. and Appl..

[52] Necas, Les Méthodes Directes en Théorie des Équations Elliptiques. Editions de l'Academie Tchecoslovaque des Sciences, Praga, 1967.

[53] Nespoli, G. On the existence of weak solutions of the exterior non-stationary problem for an incompressible nonhomogeneous viscous fluid in non-cilindrical domains. Inst. Lombardo (Rend. SC) A 130 (1960, 183-203.

[54] Okamoto, H. On the equation of nonstationary stratified fluid motion: uniquess and existence of the solutions, J. Fac. Sci. Univ. Tokyo, Sect. IA Math. 30 (1984), 615-643.

[55] Ortega, J., Rojas-Medar, M. A., On the controllability of stationary magnetomicropolar fluids, to appear in Nonlinear Dynamics, Chaos, Control and their Applications to Eng. Sc..

[56] Ortega-Torres, E. E., Rojas-Medar, M. A., On the uniqueness and regularity of the weak solution for magneto-micropolar fluid equation, Rev. Mat. Apl., v.17 (1996), 75-90.

[57] Ortega-Torres, E. E., Rojas-Medar, M. A., The initial value problem for the equations of magneto-micropolar fluid in a time-dependent domain, Mat. Contemp., 15 (1998), 259-281.

[58] Ortega-Torres, E., Rojas-Medar, M. A., Magneto-micropolar fluid motion: global existence of strong solution, publicado em Applied Analysis.

[59] Ortega-Torres, E. E., Rojas-Medar, M. A. The equations of a viscous asymmetric fluids; on a iterative approach, aceito para publicação em ZAMM.

[60] Padula, M., On the existence for non-homogeneous incompressible fluids, Rend. Circ. Mat. Palermo II, Ser. I 287 (1978), 1009-1012. 
[61] Padula, M. On the existence and uniqueness of non-homogeneous motions in exterior domains, Math. Z. 203 (1990), 581-604.

[62] Padula, M., Russo, R., A uniqueness theorem for micropolar fluid motions in unbounded regions, Bolletino U. M. I. (5) 13-A, (1976), 660-666.

[63] Pretrosyan, L. G. Some Problems of Mechanics of Fluid with Antisymetric Stress Tensor, Erevan, 1984.

[64] Rojas-Medar, M. A., On the existence of weak and strong solutions for the magneto-micropolar fluids equations in a time dependent domain, in Numerical Methods in Mechanic (Eds. C. Conca, G. N. Gatica), Pitman Research Note in Mathematics Series, vol. 371 (1997).

[65] Rojas-Medar, M. A., Boldrini, J. L., Magneto-micropolar fluid motion: Existence of weak solutions, Rev. Math. Univ. Comp. de Madrid, 11(2) (1998), 443-460.

[66] Rojas-Medar, M. A. Magneto-micropolar fluid motion: Existence and uniqueness of strong solutions, Math. Nach., 188 (1997), 301-319.

[67] Rojas-Medar, M. A. Algumas Questões Matemáticas das Equações dos Fluidos Não-Homogêneos, Minicurso, 41ำ Seminário Brasileiro de Análise.

[68] Salvi, R., On the existence of weak solutions of non-linear mixed problems for the dependent domain, C. M. U. C. 26 (1985), 185-199.

[69] Salvi, R., The exterior non-stationary problem for the Navier-Stokes equations in regions with moving boudaries, J. Math. Soc. Japan, v. 42, 3 (1990), 495-509.

[70] Salvi, R., Soluzioni periodiche delle equazioni dei fluidi viscosi incomprimibli non omogenei, Riv. Mat. Parma, 4 (1983), 241-251.

[71] Salvi, R., Diziquazioni variazionali per i fluidi viscosi incomprimibili non omogenei, Riv. Mat. Parma, 4 (1982), 453-466.

[72] Salvi, R., On the existence of weak solutions of boundary value problems in a diffusion model for an inhomogeneous liquid in regions with moving boundary, Portugaliae Mathematica, v. 43, 2 (1985-1986), 213-233. 
[73] Salvi, R., The equations of viscous incompressible nonhomogeneous fluid: on the existence and regularity, J. Australian Math. Soc., Series B - Applied Math. 33(1) (1991), 94-110.

[74] Santos, M. M. -Stationary solution of the Navier-Stokes equations in a 2D bounded domain for incompressible flow with discontinuous density, pre-printer, IMECC-UNICAMP, 1998.to appear in ZAMP.

[75] Serrin, J., Mathematical Principles of Classical Fluid Mechanics, Handbuch der Physik, vol. VIII/1 (1959), 125-262.

[76] Serrin, J., A note on the existence of periodic solutions of the Navier-Stokes equations, Arch. Rat. Mech. 3 (1959), 120-122.

[77] Serrin, J., On the interior regularity of weak solutions of the Navier-Stokes equations, Arch. Rat. Mech. Anal., 9 (1962), 187-195.

[78] Simon, J., Nonhomogeneous viscous incompressible fluid: Existence of velocity, density and pressure, SIAM J. Math., 21 (1990), 1093-1117.

[79] Simon, J., Existencia de Solución del Problema de Navier-Stokes con Densidad Variable, Univ. de Sevilla Facultad de Matemáticas, Depto. de Análisis Matemático, 1994.

[80] Simon, J., Compact sets in the space $L^{p}(O ; T ; B)$, Annali Mat. Pura Appl., Serie IV, v.146 (1987), 65-96.

[81] Simon, J., Sobolev, Besov and Nikolskii spaces: imbeddings and comparisons for vector valued spaces on a interval, Annali Mat. Pura Appl., Serie IV, 1989.

[82] Solonnikov, V. A., On the boundary value problems for linear parabolic system of general type, Trudy Math. Inst. Steklev, v.83 (1965), 1-162.

[83] Solonnikov, V. A., Solvability of the initial-boundary-value problem for the equations of motion of a viscous compressible fluid, J. Soviet Math., 14 (1980), $1120-1133$.

[84] Takeshita, A. On the Reprodutive Property of the 2-dimensional Navier-Stokes Equations, J. Fac. Sci. Univ. Tokyo sect. I, 16 (1969), 297-311.

[85] Temann, R., Navier-Stokes Equations, Theory and Numerical Analysis, NorthHolland, Amsterdam, 1979. 
[86] von Whahl, W., The Equations of Navier-Stokes and Abstract Parabolic Equations, Aspects of Math., 58, Vieweg, Braunschweig-Wiesbaden, 1985.

[87] Yudovič, V. I., Periodic motions of a viscous incompressible fluid, Soviet Math. Dokl. 1 (1960), 168-172. 